\title{
Enantioselective Synthesis of Cyclohexenol Derivatives from $\gamma$-Aryl-substituted Enals via an Organocatalyzed Three-Component Reaction
}

Debashis Majee, Satish Jakkampudi, Hadi D. Arman, and John C.-G. Zhao*

Department of Chemistry, University of Texas at San Antonio

\section{Supporting Information}

\section{Table of Contents}

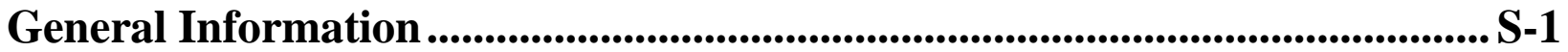

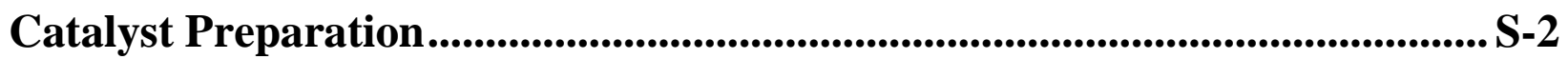

Detailed Experimental Procedures......................................................................... S-3

Detailed Catalyst Screening and Condition Optimizations ................................ S-5

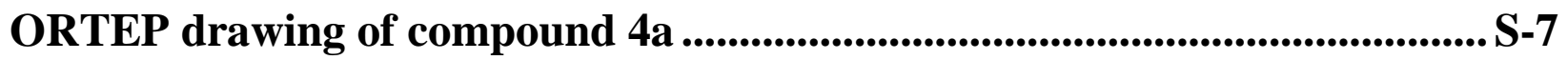

Alternative Reaction Mechanism ............................................................... S-8

Compound Characterization Data .................................................................................. S-9

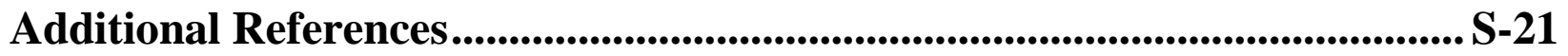

Copy of ${ }^{1} \mathrm{H}$ and ${ }^{13} \mathrm{C}$ NMR Spectra ............................................................................. S-22

Copy of HPLC Chromatograms.................................................................................. S-41 


\section{General Information}

All reactions were carried out in closed vial and monitored by TLC and the products were visualized by UV detection. Flash column chromatography was performed with silica gel (32-63 $\mu) .{ }^{1} \mathrm{H}$ and ${ }^{13} \mathrm{C}$ NMR spectra were recorded on a $500 \mathrm{MHz}\left(125 \mathrm{MHz}\right.$ for $\left.{ }^{13} \mathrm{C} \mathrm{NMR}\right)$ or a $300 \mathrm{MHz}$ spectrometer (75 MHz for $\left.{ }^{13} \mathrm{C} \mathrm{NMR}\right)$. The following abbreviations were used to designate chemical shift mutiplicities: $\mathrm{s}=$ singlet, $\mathrm{d}=$ doublet, $\mathrm{t}=$ triplet, $\mathrm{q}=$ quartet, $\mathrm{m}=$ multiplet, $\mathrm{br}=$ broad. All first-order splitting patterns were assigned on the basis of the appearance of the multiplet. Splitting patterns that could not be easily interpreted are designated as multiplet (m) or broad (br). FTIR spectra were measured on a Bruker Vector 22 instrument. Enantiomeric excesses (ee) were determined by HPLC analysis using a Shimadzu instrument with chiral HPLC columns. ChiralPak IB, IC, and ID columns $(4.6 \mathrm{~mm} \times 250 \mathrm{~mm})$ were purchased from Daicel Chemical Industries. Melting points were recorded on MEL-TEMP melting point apparatus in open capillaries and uncorrected. HRMS analyses were conducted at the RCMI Proteomics and Protein Biomarkers Core Facility of the Department of Chemistry, UTSA.

Materials: All the $\alpha, \beta$-unsaturated aldehydes were prepared by following the literature procedure. ${ }^{12}$ All the nitroalkenes were either purchased from commercial sources or synthesized by following known literature procedures. ${ }^{13}$ All catalysts, except for $\mathbf{5 e}, \mathbf{5 f}, \mathbf{5 g}$, and $\mathbf{5 y}$, are known compounds and were prepared by following the known procedures. ${ }^{14}$ Tetrahydrofuran was dried over sodium metal under argon atmosphere and distilled before prior to use. 


\section{Catalyst Preparation}

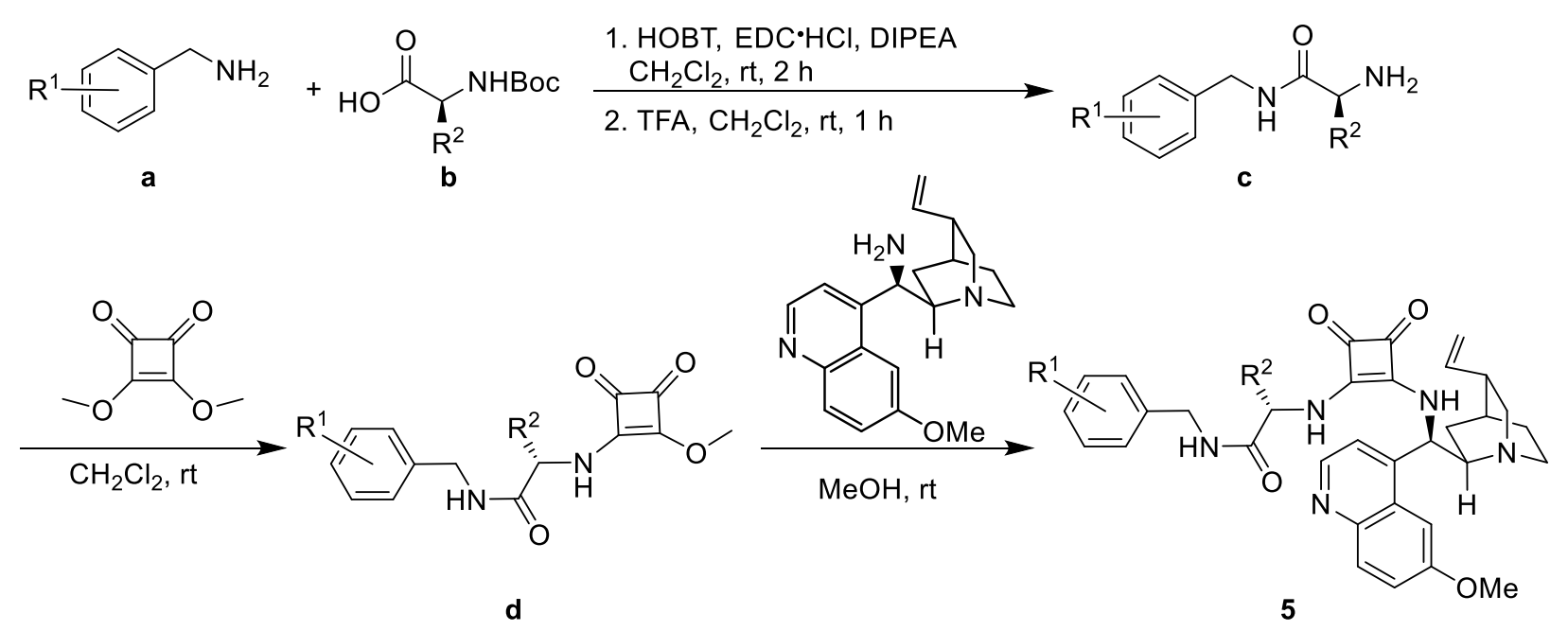

Scheme S-1. Synthesis of the squaramide catalysts 5 .

Preparation of intermediate c: Intermediate c was prepared according to the literature procedure. ${ }^{15}$ In a 250-mL round-bottom flask, the Boc-protected amino acid b (5.0 mmol), hydroxybenzotriazole (HOBT, $676 \mathrm{mg}, 5.0 \mathrm{mmol}, 1.0$ equiv.), and EDC hydrochloride (959 mg, 5.0 mmol, 1.0 equiv.) were suspended in dry $\mathrm{CH}_{2} \mathrm{Cl}_{2}(35 \mathrm{~mL})$ at room temperature. After 2 min., DIPEA (1.3 g, $10 \mathrm{mmol}, 2.0$ equiv.) was added, which was followed immediately by the benzylamine derivative a (5.0 mmol, 1.0 equiv.). The reaction mixture was allowed to stir further for $2 \mathrm{~h}$ at the same temperature. During the reaction progress, due to the consumption of HOBT, the mixture changed from turbid to a clear solution. The reaction was then quenched by adding $1 \mathrm{~N}$ $\mathrm{HCl}(20 \mathrm{~mL})$ and the mixture was extracted with $\mathrm{CH}_{2} \mathrm{Cl}_{2}(3 \times 30 \mathrm{~mL})$. The combined organic layers were dried over anhydrous $\mathrm{Na}_{2} \mathrm{SO}_{4}$ and concentrated under reduced pressure. The resulting colorless liquid was further dissolved in $\mathrm{CH}_{2} \mathrm{Cl}_{2}(25 \mathrm{~mL})$ and treated with trifluoroacetic acid (75 mmol, 15 equiv.) under stirring. After $1 \mathrm{~h}$ the reaction mixture was diluted with $\mathrm{CH}_{2} \mathrm{Cl}_{2}(30 \mathrm{~mL})$ and quenched with chilled saturated solution of sodium carbonate $(50 \mathrm{~mL})$. After neutralization by adding more saturated sodium carbonate solution, the aqueous layer was extracted with $\mathrm{CH}_{2} \mathrm{Cl}_{2}$ (3 $\times 20 \mathrm{~mL}$ ), and the combined organic layers were dried over anhydrous $\mathrm{Na}_{2} \mathrm{SO}_{4}$. The solvent was removed under reduced pressure to give a white solid that was used without further purification. 
Synthesis of intermediate $\mathbf{d}$ : The crude intermediate c $(5.0 \mathrm{mmol})$ was dissolved in $\mathrm{CH}_{2} \mathrm{Cl}_{2}(25$ $\mathrm{mL})$ and dimethylsquarate $(710.6 \mathrm{mg}, 5.0 \mathrm{mmol})$ was added portion wise over $10 \mathrm{~min}$. at room temperature. The mixture was further stirred for $72 \mathrm{~h}$ to complete the reaction (monitored by TLC). The solvent was then removed under reduced pressure and the crude product was purified by in flash column chromatography using $98: 2 \mathrm{CH}_{2} \mathrm{Cl}_{2} / \mathrm{MeOH}$ as the eluent to give the intermediate $\mathbf{d}$ $(63-72 \%)$.

Synthesis of 5: In an oven-dried round-bottom flask, the quinine- or quinidine-derived 9-amino(9deoxy)cinchona alkaloid $(255.5 \mathrm{mg}, 0.79 \mathrm{mmol})$ was dissolved in dry methanol $(10 \mathrm{~mL})$ at room temperature. To that solution, $\mathbf{d}(0.79 \mathrm{mmol})$ was added in one portion and the mixture was further stirred for 48-90 h. During the progress, a white precipitate was observed. The precipitate was filtered and washed with cold $\mathrm{MeOH} /$ hexane (1:4), which gave the pure catalyst $\mathbf{5}$. The filtrate was further concentrated and purified by silica gel flash column chromatography using $\mathrm{MeOH} / \mathrm{CH}_{2} \mathrm{Cl}_{2}$ (0:100 to 5:95) as a mobile phase to give an additional portion of the catalyst. Both the portions were combined for catalytic use (total yield 55-60\%).

\section{Detailed Experimental Procedures}

General procedure for the three-component reaction: To a stirred solution of the appropriate nitroalkene (0.60 mmol, 3.0 equiv.) and the squaramide catalyst $\mathbf{5 f}$ ( $24.9 \mathrm{mg}, 0.040 \mathrm{mmol}, 20$ mol \%) in freshly distilled THF $(0.7 \mathrm{~mL})$ at room temperature, the $\gamma$-aryl- $\alpha, \beta$-unsaturated aldehyde $(0.20 \mathrm{mmol})$ was added. The mixture was further stirred at room temperature for $72 \mathrm{~h}$ (the progress

of the reaction was monitored by TLC). After the completion of the reaction, the solvent was evaporated in a rotary evaporator under the reduced pressure and the crude product obtained was purified by flash column chromatography using 90:10 to 95:5 hexane/EtOAc as an eluent.

\section{$1.0 \mathrm{mmol}-\mathrm{scale}$ reaction}


To a stirred solution of trans- $\beta$-nitrostyrene (2a, $447.5 \mathrm{mg}, 3.0 \mathrm{mmol}, 3.0$ equiv.) and the squaramide catalyst $\mathbf{5 f}(124.4 \mathrm{mg}, 0.20 \mathrm{mmol}, 0.2$ equiv) in freshly distilled THF ( $3.5 \mathrm{~mL})$ at room temperature, 4-phenyl-2-butenal (1a, $146.2 \mathrm{mg}, 1.0 \mathrm{mmol}$ ) was added. The mixture was further stirred at room temperature for $72 \mathrm{~h}$ (the progress of the reaction was monitored by TLC). After the completion of the reaction, the solvent was evaporated in a rotary evaporator under reduced pressure and the crude product obtained was purified by flash column chromatography using 95:5 hexanes/EtOAc as an eluent to give $\mathbf{4 a}$ as white color solid $(310.5 \mathrm{mg}$, 70\% yield, 87:13 dr, 99\% ee).

\section{Acetylation of the adduct $4 a$}

To a solution of the adduct $4 \mathbf{a}(44.5 \mathrm{mg}, 0.10 \mathrm{mmol})$ in $\mathrm{CH}_{2} \mathrm{Cl}_{2}(3.0 \mathrm{~mL})$, acetic anhydride $(0.10$ $\mathrm{mL}, 1.0 \mathrm{mmol})$ and DMAP $(2.5 \mathrm{mg}, 0.020 \mathrm{mmol})$ were added at $0{ }^{\circ} \mathrm{C}$ and the reaction mixture was further stirred for $30 \mathrm{~min}$ at room temperature. After the completion the reaction, the solvent was evaporated under reduced pressure, and the crude product was purified by flash chromatography using 93:7 hexanes/EtOAc as an eluent to give $\mathbf{6}$ as white solid (38.7 mg, 80\% yield).

\section{Oxidation of the adduct $4 a$}

To a solution of the adduct $4 \mathbf{a}(44.5 \mathrm{mg}, 0.10 \mathrm{mmol})$ in $\mathrm{CH}_{2} \mathrm{Cl}_{2}(3.0 \mathrm{~mL}), 100 \mathrm{mg}$ Celite was suspended and then PCC (64.7 $\mathrm{mg}, 0.30 \mathrm{mmol})$ were added at room temperature and the reaction mixture was further stirred for $12 \mathrm{~h}$ at the same temperature. After the completion the reaction, the mixture was filtered through a Celite pad and the crude product was purified by flash chromatography using 92:8 hexane/EtOAc as an eluent to give 7 as white solid. (37.6 mg, 85\% yield). 


\section{Detailed Catalyst Screening and Condition Optimizations}

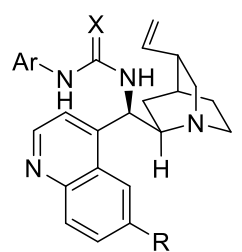

5a: $X=S ; R=O M e$ 5h: $X=S ; R=O i-P r$ 5i: $X=S ; R=H$
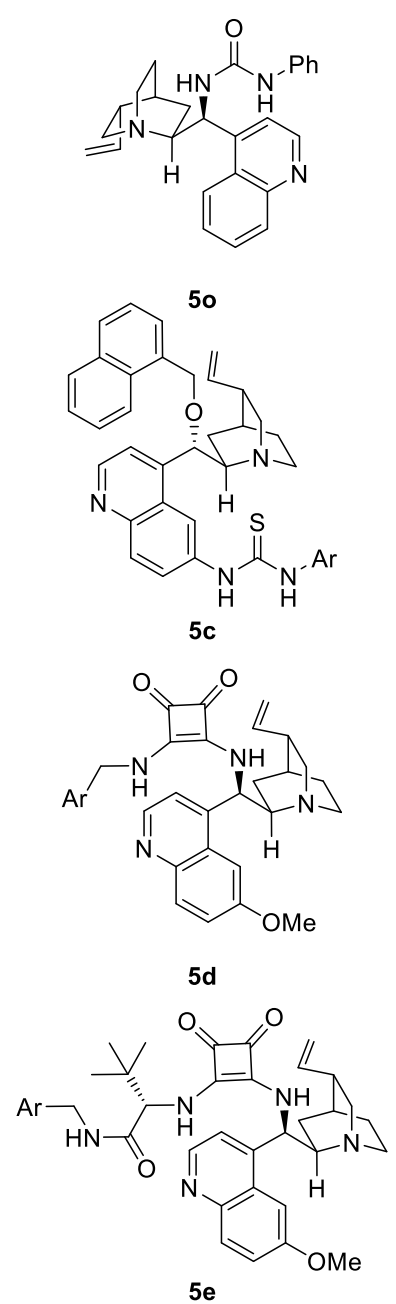

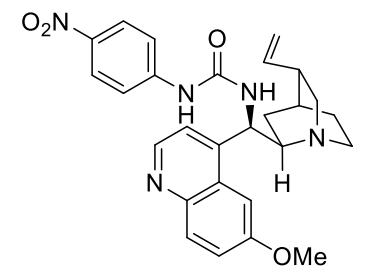

$5 \mathbf{j}$
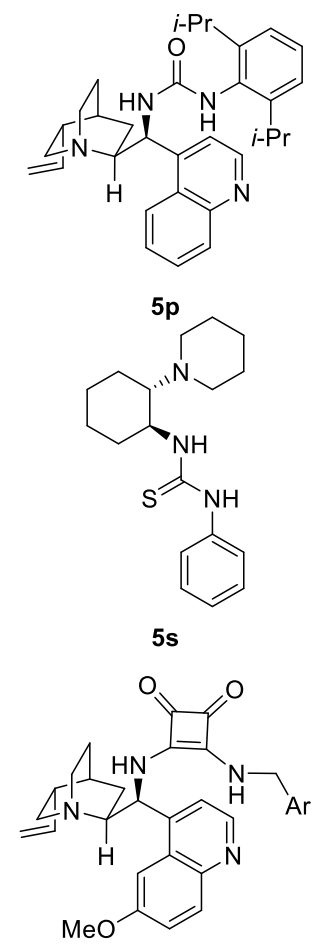

$5 v$

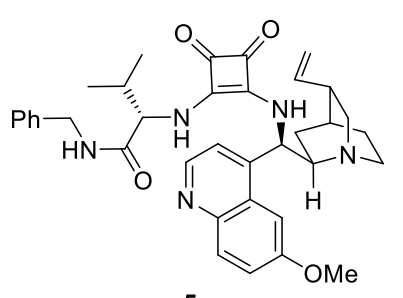

$5 y$

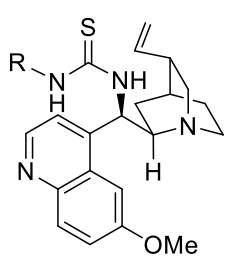

5k: $\mathrm{R}=\mathrm{PhSO}_{2}$

5I: $R={ }_{A C O}^{A c O} \frac{\sqrt{O A} y_{2}}{O A C}$
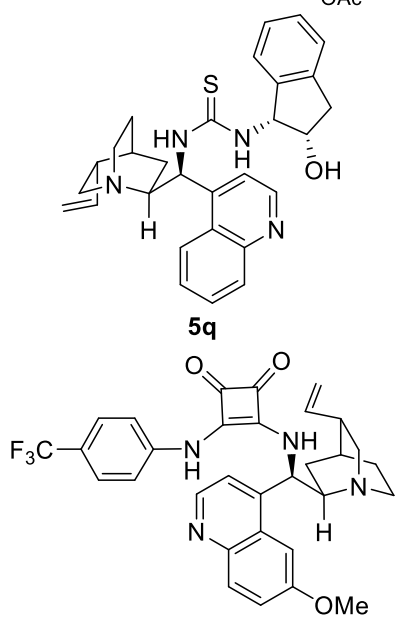

$5 \mathbf{t}$
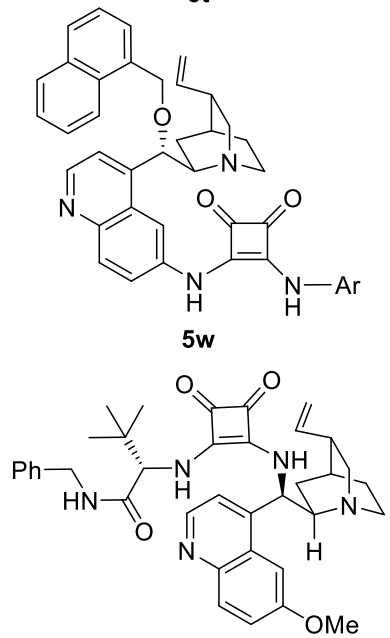

$5 f$

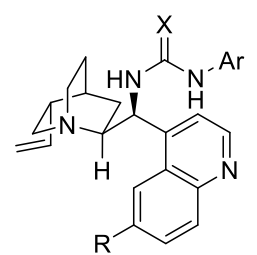

5b: $X=S ; R=O M e$

$5 \mathrm{~m}: X=S ; R=H$

$5 n: X=O ; R=H$
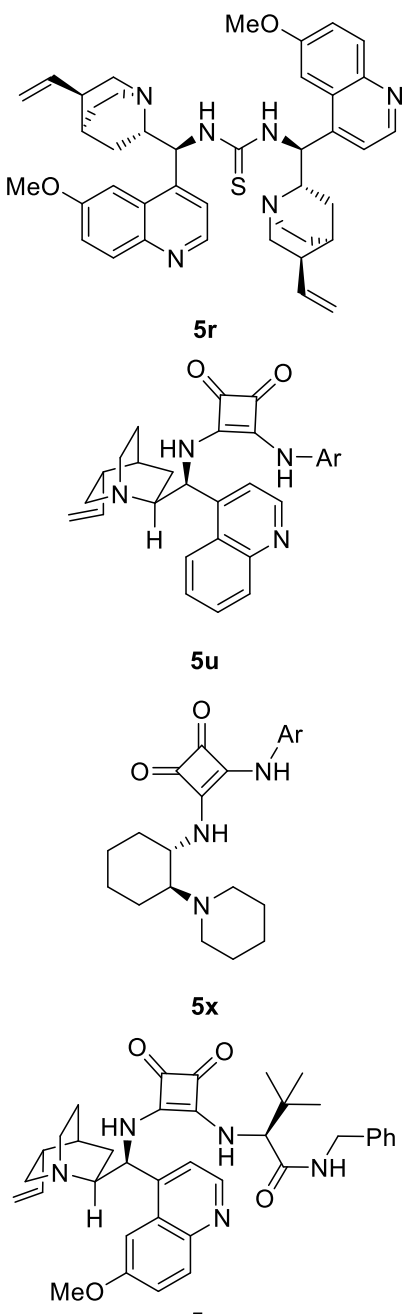

$5 g$

Figure S-1. Structure of catalyst used in the screening 
Table S-1: Screening of the catalysts ${ }^{a}$

\begin{tabular}{ccccc} 
& & & \\
& & & & \\
& & & & \\
\hline
\end{tabular}

${ }^{a}$ Unless otherwise noted, all reactions were carried out with $\mathbf{1 a}(0.20 \mathrm{mmol})$ and $\mathbf{2 a}(0.6 \mathrm{mmol})$ in dry THF $(0.7 \mathrm{~mL})$ at room temperature for $72 \mathrm{~h}$, using $5(20 \mathrm{~mol} \%)$ as the catalyst. ${ }^{b}$ Yield of the isolated product. ${ }^{c}$ Diastereomeric ratio was determined by ${ }^{1} \mathrm{H}$ NMR analysis of the crude reaction mixture. ${ }^{d}$ Determined by chiral HPLC analysis. ${ }^{e}$ The opposite enantiomer was obtained as the major product. ${ }^{f}$ The catalyst loading was $10 \mathrm{~mol} \%$. 


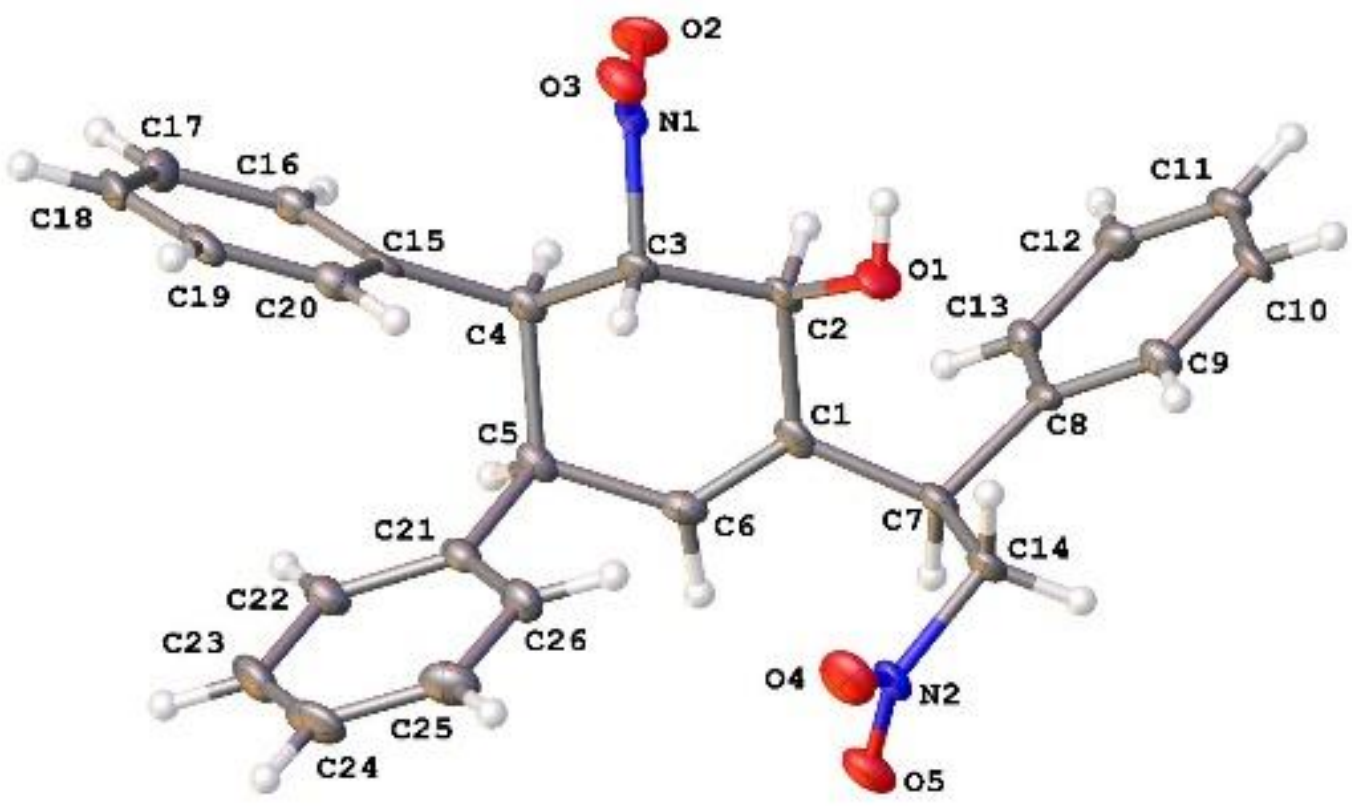

Figure S-2. ORTEP drawing of compound 4a 


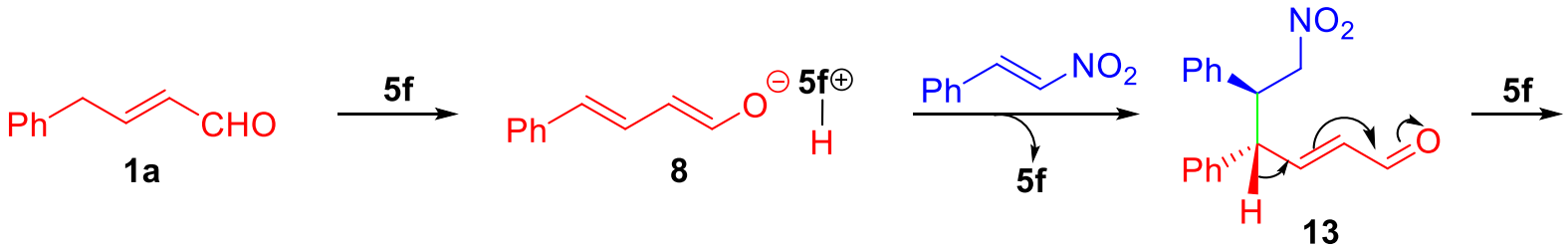<smiles></smiles>

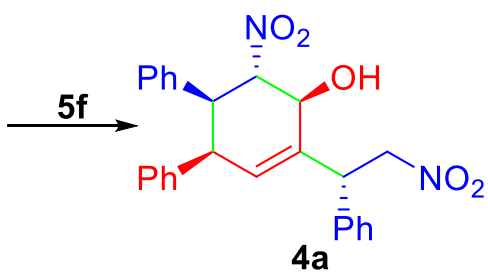

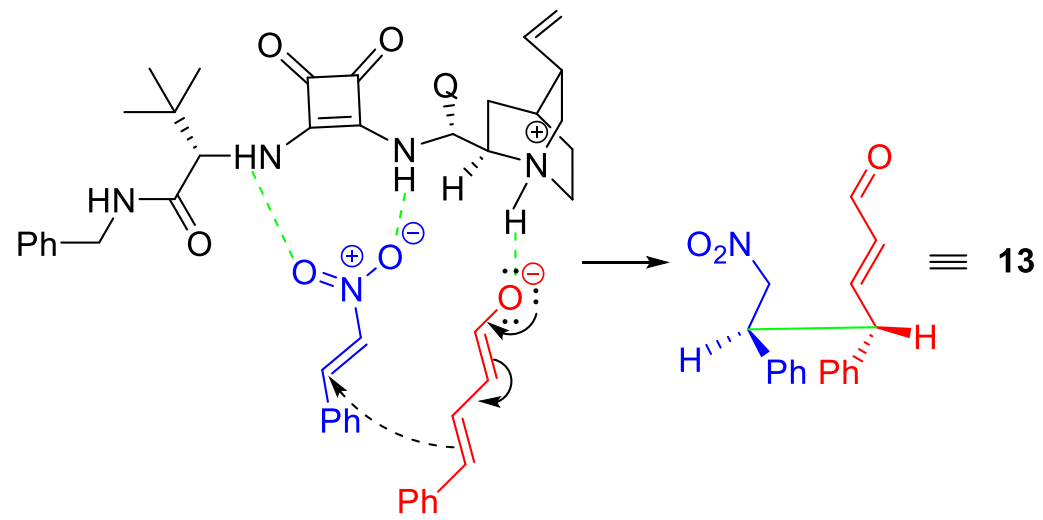

16<smiles>CC(C)(C)[P+](=O)[O-]</smiles>

Scheme S-2. Alternative reaction mechanism for the three-component reaction 


\section{Compound Characterization Data}

\section{3- $\left\{(S)-N^{\prime}-[3,5-B i s(\right.$ trifluoromethyl)benzyl]-3,3-dimethylbutanamid-2-ylamino\}-4-\{[(9R)-6'- methoxycinchonan-9-yl]amino\}-3-cyclobutene-1,2-dione (5e)}

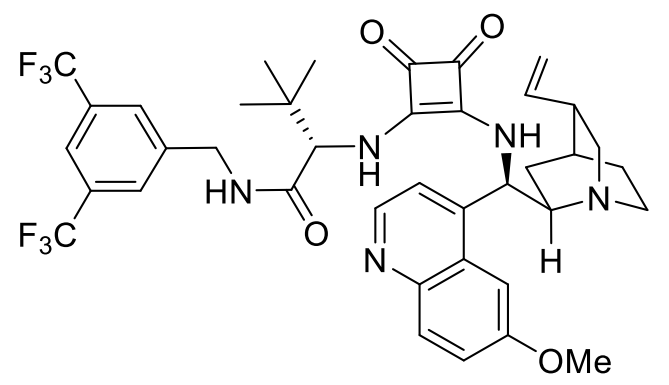

The crude product was purified by silica gel flash column chromatography using $\mathrm{MeOH} / \mathrm{CH}_{2} \mathrm{Cl}_{2}(0: 100$ to 5:95) to get 5e as a white solid, $2.01 \mathrm{~g}, 55 \%$ yield, m.p. 178-180 ${ }^{\circ} \mathrm{C} ;{ }^{1} \mathrm{H} \mathrm{NMR}\left(500 \mathrm{MHz}, \mathrm{CDCl}_{3}\right) \delta: 8.88(\mathrm{~s}, 2 \mathrm{H}), 8.56(\mathrm{~s}$, 2H), $8.06(\mathrm{~d}, J=9.2 \mathrm{~Hz}, 1 \mathrm{H}), 7.95(\mathrm{~s}, 1 \mathrm{H}), 7.70(\mathrm{~s}, 1 \mathrm{H})$, 7.44-7.40 (m, 2H), $7.27(\mathrm{~s}, 1 \mathrm{H}), 7.12(\mathrm{br}, 1 \mathrm{H}), 6.51$ (br, 1H), $5.97(\mathrm{~d}, J=14.3 \mathrm{~Hz}, 1 \mathrm{H}), 5.29$ (d, $J=16.9 \mathrm{~Hz}, 1 \mathrm{H}), 5.13(\mathrm{~d}, J=10.0 \mathrm{~Hz}, 1 \mathrm{H}), 4.47(\mathrm{~s}, 2 \mathrm{H})$, 4.14 (br, 1H), 3.89 (s, 3H), 3.42 (br, 2H), 3.06 (s, 3H), 2.41 (s, 1H), 1.92 (s, 3H), 1.72 (s, 3H), 1.16-1.04 (m, 2H), 0.85 (s, 9H). ${ }^{13} \mathrm{C}$ NMR (125 MHz, $\left.\mathrm{CDCl}_{3}\right) \delta: 183.0,181.7,171.8,169.2,165.5$, $159.0,148.1,145.2,144.7,141.0,140.8,132.2,132.1\left(\mathrm{q}, J_{\mathrm{C}-\mathrm{F}}=33.7 \mathrm{~Hz}\right), 125.8,125.3\left(\mathrm{q}, J_{\mathrm{C}-\mathrm{F}}=\right.$ 273.0 Hz), 122.7, 121.0, 119.8 (br.), 119.5, 115.1, 101.9, 64.8, 59.3, 56.0, 53.6, 49.5, 47.1, 42.1, 39.7, 35.6, 28.1, 27.4, 26.5, 26.1. $v_{\max }\left(\right.$ neat, $\left.\mathrm{cm}^{-1}\right): 3234,2936,1660,1622,1582,1509,1434$, $1379,1349,1275,1243,1170,1128,1021$. HRMS (ESI) $m / z$ calcd for $\mathrm{C}_{39} \mathrm{H}_{42} \mathrm{~F}_{6} \mathrm{~N}_{5} \mathrm{O}_{4}{ }^{+}([\mathrm{M}+\mathrm{H}])$ : 758.3136; found: 758.3146.

\section{3-[(S)- $N^{\prime}$-Benzyl-3,3-dimethylbutanamid-2-ylamino]-4-\{[(9R)-6'-methoxycinchonan-9-} yl]amino\}-3-cyclobutene-1,2-dione (5f)

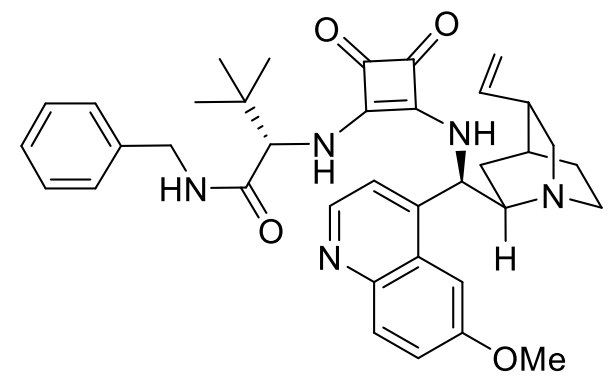

The crude product was purified by silica gel flash column chromatography using $\mathrm{MeOH} / \mathrm{CH}_{2} \mathrm{Cl}_{2}$ (0:100 to 3:97) to get 5f as a white solid, $1.86 \mathrm{~g}, 60 \%$ yield, m.p. 190-192 ${ }^{\circ} \mathrm{C} ;{ }^{1} \mathrm{H}$ NMR (500 MHz, $\left.\mathrm{CDCl}_{3}\right) \delta: 8.70(\mathrm{br}, 1 \mathrm{H}), 8.54-8.62(\mathrm{~m}$, 1H), 8.42 (br, 1H), 8.05 (d, $J=9.1 \mathrm{~Hz}, 1 \mathrm{H}), 7.98$ (s, 1H), $7.81(\mathrm{~s}, 1 \mathrm{H}), 7.43$ (d, $J=9.3 \mathrm{~Hz}, 1 \mathrm{H}), 7.26$ (br, 1H), 7.08$7.06(\mathrm{~m}, 3 \mathrm{H}), 6.84-6.83(\mathrm{~m}, 2 \mathrm{H}), 6.51(\mathrm{br}, 1 \mathrm{H}), 5.90\left(\mathrm{ddd}, J_{1}=17.0 \mathrm{~Hz}, J_{2}=10.5, J_{3}=6.2 \mathrm{~Hz}\right.$, $1 \mathrm{H}), 5.20(\mathrm{~d}, J=17.1 \mathrm{~Hz}, 1 \mathrm{H}), 5.05(\mathrm{~d}, J=10.3 \mathrm{~Hz}, 1 \mathrm{H}), 4.54(\mathrm{~d}, J=9.5 \mathrm{~Hz}, 1 \mathrm{H}), 4.13(\mathrm{~s}, 2 \mathrm{H})$, $3.92(\mathrm{~s}, 3 \mathrm{H}), 3.78-3.75(\mathrm{~m}, 1 \mathrm{H}), 3.36(\mathrm{~s}, 1 \mathrm{H}), 3.24(\mathrm{~s}, 1 \mathrm{H}), 2.97-2.93(\mathrm{~m}, 2 \mathrm{H}), 2.30-2.28(\mathrm{~m}, 2 \mathrm{H})$, 1.64-1.57 (m, 3H), $1.11(\mathrm{~s}, 1 \mathrm{H}), 0.99$ (s, 1H), $0.81(\mathrm{~s}, 9 \mathrm{H}) .{ }^{13} \mathrm{C} \mathrm{NMR}\left(125 \mathrm{MHz}, \mathrm{CDCl}_{3}\right) \delta: 183.0$, 
182.1, 170.8, 168.9, 166.1, 158.8, 148.2, 145.1, 140.8, 137.7, 132.0, 128.8, 128.4, 127.6, 122.7, 119.9, 115.1, 101.9, 64.9, 59.3, 56.1, 53.3, 49.4, 47.2, 43.4, 39.8, 35.9, 28.2, 27.3, 26.7, 25.8, 23.0, 14.5, $12.4 v_{\max }\left(\right.$ neat, $\mathrm{cm}^{-1}$ ): 3301, 2932, 1795, 1652, 1621, 1582, 1509, 1432, 1368, 1227, 1128. HRMS (ESI) $\mathrm{m} / z$ calcd for $\mathrm{C}_{37} \mathrm{H}_{44} \mathrm{~N}_{5} \mathrm{O}_{4}\left([\mathrm{M}+\mathrm{H}]^{+}\right)$: 622.3388; found: 622.3390 .

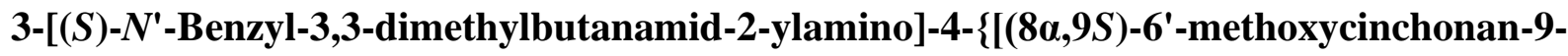
yl]amino\}-3-cyclobutene-1,2-dione (5g)

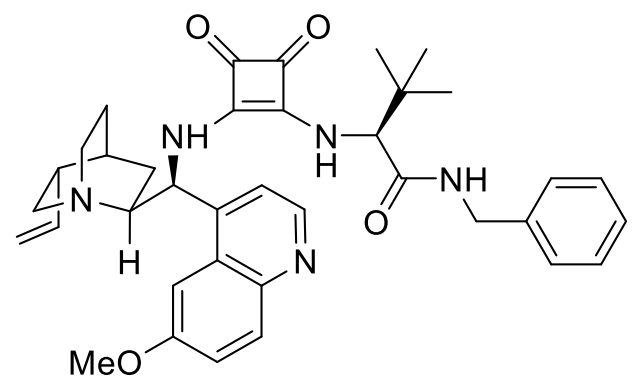

The crude product was purified by silica gel flash column chromatography using $\mathrm{MeOH} / \mathrm{CH}_{2} \mathrm{Cl}_{2}$ (0:100 to 4:96) to get $\mathbf{5 g}$ as a white solid, $1.71 \mathrm{~g}, 55 \%$ yield, m.p. 182$184^{\circ} \mathrm{C} ;{ }^{1} \mathrm{H}$ NMR $\left(500 \mathrm{MHz}, \mathrm{CDCl}_{3}\right) \delta: 8.45(\mathrm{br}, 1 \mathrm{H}), 8.01-$ $7.98(\mathrm{~m}, 3 \mathrm{H}), 7.57(\mathrm{~s}, 2 \mathrm{H}), 7.38-7.36(\mathrm{~m}, 1 \mathrm{H}), 7.02-6.94$ (m, 4H), 6.28 (br, 1H), 5.89 (br, 1H), 5.03-4.93 (m, 2H), $4.58(\mathrm{~s}, 1 \mathrm{H}), 3.94-3.88(\mathrm{~m}, 5 \mathrm{H}), 3.40(\mathrm{~s}, 1 \mathrm{H}), 3.24(\mathrm{t}, J=10 \mathrm{~Hz}, 1 \mathrm{H}), 2.85-2.76(\mathrm{~m}, 2 \mathrm{H}), 2.29$ (d, $J=7.2 \mathrm{~Hz}, 1 \mathrm{H}), 1.64-1.44(\mathrm{~m}, 4 \mathrm{H}), 0.88(\mathrm{br}, 9 \mathrm{H}) .{ }^{13} \mathrm{C} \mathrm{NMR}\left(125 \mathrm{MHz}, \mathrm{CDCl}_{3}\right) \delta: 183.3,170.8$, 168.8, 167.2, 159.0, 148.2, 145.1, 144.8, 142.1, 137.9, 132.2, 129.4, 128.7, 127.6, 122.6, 120.0, 114.9, 102.0, 64.9, 56.2, 53.9, 43.5, 41.2, 40.1, 35.4, 28.3, 26.5. $v_{\max }$ (neat, $\left.\mathrm{cm}^{-1}\right): 33264,2942$, $1657,1621,1581,1524,1454,1367,1259,1228,1176,1132,1028$. HRMS (ESI) $\mathrm{m} / \mathrm{z}$ calcd for $\mathrm{C}_{37} \mathrm{H}_{44} \mathrm{~N}_{5} \mathrm{O}_{4}\left([\mathrm{M}+\mathrm{H}]^{+}\right)$: 622.3388; found: 622.3401 .

\section{3-[(S)-N'-Benzyl-3-methylbutanamid-2-ylamino]-4-\{[(9R)-6'-methoxycinchonan-9-} yl]amino\}-3-cyclobutene-1,2-dione (5y)

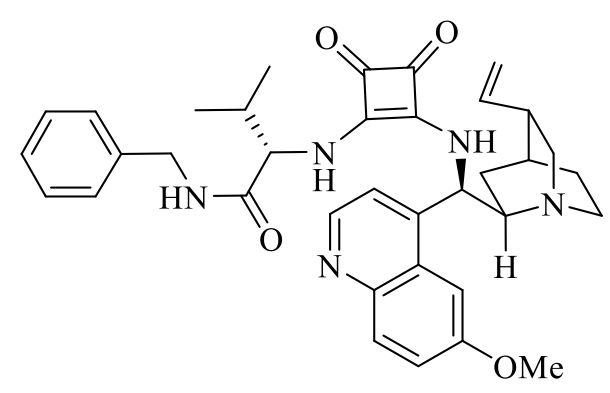

The crude product was purified by silica gel flash column chromatography using $\mathrm{MeOH} / \mathrm{CH}_{2} \mathrm{Cl}_{2}$ (0:100 to 4:96) to get 5y as a white solid, $1.67 \mathrm{~g}, 58 \%$ yield, m.p. $170-172{ }^{\circ} \mathrm{C} ;{ }^{1} \mathrm{H}$ NMR (500 MHz, $\left.\mathrm{CDCl}_{3}\right) \delta: 8.75(\mathrm{br}, 2 \mathrm{H}), 8.50(\mathrm{~s}, 1 \mathrm{H}), 8.09$ $8.04(\mathrm{~m}, 2 \mathrm{H}), 7.81(\mathrm{~s}, 1 \mathrm{H}), 7.46(\mathrm{~d}, J=9.3 \mathrm{~Hz}, 2 \mathrm{H}), 7.11(\mathrm{~s}$, $3 \mathrm{H}), 6.88(\mathrm{~s}, 2 \mathrm{H}), 6.54(\mathrm{~s}, 1 \mathrm{H}), 5.93\left(\mathrm{td}, J_{1}=11.0 \mathrm{~Hz}, J_{2}=\right.$ $\left.10.3 \mathrm{~Hz}, J_{3}=5.7 \mathrm{~Hz}, 1 \mathrm{H}\right), 5.25(\mathrm{~d}, J=17.2 \mathrm{~Hz}, 1 \mathrm{H}), 5.09(\mathrm{~d}, J=10.4 \mathrm{~Hz}, 1 \mathrm{H}), 4.60(\mathrm{~s}, 1 \mathrm{H}), 4.10-$ $3.94(\mathrm{~m}, 6 \mathrm{H}), 3.72(\mathrm{~s}, 1 \mathrm{H}), 3.35(\mathrm{~s}, 1 \mathrm{H}), 3.22(\mathrm{~s}, 1 \mathrm{H}), 3.03-3.00(\mathrm{~m}, 2 \mathrm{H}), 2.33(\mathrm{~d}, J=8.1 \mathrm{~Hz}, 1 \mathrm{H})$, 1.94-1.86 (m, 2H), 1.67-1.62 (m, 3H), 1.14 (s, 1H), 1.07-0.89 (m, 2H), 0.81 (d, $J=6.4 \mathrm{~Hz}, 3 \mathrm{H})$, 
$0.73(\mathrm{~d}, J=5 \mathrm{~Hz}, 3 \mathrm{H}) .{ }^{13} \mathrm{C} \mathrm{NMR}\left(125 \mathrm{MHz}, \mathrm{CDCl}_{3}\right) \delta: 183.2,181.55,171.7,169.2,165.6,158.7$, 148.2, 145.3, 145.1, 140.9, 137.6, 132.0, 128.8, 128.3, 127.6, 127.4, 122.8, 119.9, 115.0, 101.9, 63.0, 59.2, 56.0, 53.4, 49.4, 47.1, 43.2, 39.8, 33.3, 28.2, 27.3, 25.8, 18.9, 18.7, 12.3. $v_{\max }$ (neat, $\mathrm{cm}^{-}$ $\left.{ }^{1}\right): 3232,2933,1655,1621,1509,1452,1367,1260,1241,1228,1159,1080,1027$. HRMS (ESI) $m / z$ calcd for $\mathrm{C}_{36} \mathrm{H}_{42} \mathrm{~N}_{5} \mathrm{O}_{4}{ }^{+}([\mathrm{M}+\mathrm{H}]): 608.3231$; found: 608.3249 .

(1'R,2'S,3' $S, 4$ ' $S)-3$ '-Nitro-5'-[(R)-2-nitro-1-phenylethyl]-1',2',3',4'-tetrahydro-[1,1':2',1'"terphenyl]-4'-ol (4a)

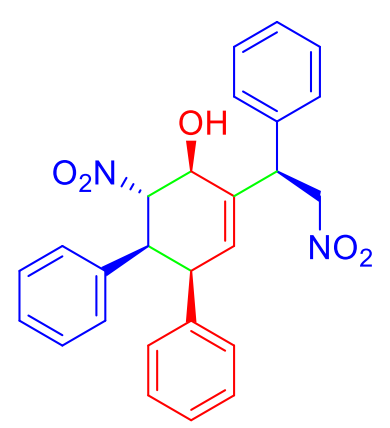

The crude material was purified by flash column chromatography using hexanes/EtOAc (95:5) as an eluent to get $\mathbf{4 a}$ as a white solid, $71 \mathrm{mg}, 80 \%$ yield, m.p. $140-142{ }^{\circ} \mathrm{C} ;{ }^{1} \mathrm{H} \mathrm{NMR}\left(500 \mathrm{MHz}, \mathrm{CDCl}_{3}\right) \delta: 7.46-7.31(\mathrm{~m}, 5 \mathrm{H})$, 7.21-7.19 (m, 1H), 7.17-7.09 (m, 3H), 7.05 (t, $J=7.4$ Hz, 2H), 6.61-6.55 (m, 4H), $6.02(\mathrm{~d}, J=4.2 \mathrm{~Hz}, 1 \mathrm{H}), 5.24\left(\mathrm{dd}, J_{1}=12.3 \mathrm{~Hz}, J_{2}=9.7 \mathrm{~Hz}, 1 \mathrm{H}\right)$, $5.11\left(\mathrm{dd}, J_{1}=12.0 \mathrm{~Hz}, J_{2}=8.5 \mathrm{~Hz}, 1 \mathrm{H}\right), 4.95\left(\mathrm{dd}, J_{1}=12.2 \mathrm{~Hz}, J_{2}=6.9\right.$ $\mathrm{Hz}, 1 \mathrm{H}), 4.80(\mathrm{t}, J=8.0 \mathrm{~Hz}, 1 \mathrm{H}), 4.63\left(\mathrm{dd}, J_{1}=9.6 \mathrm{~Hz}, J_{2}=7.0 \mathrm{~Hz}, 1 \mathrm{H}\right)$, 3.79-3.76 (m, 2H), $2.63(\mathrm{~d}, J=7.5 \mathrm{~Hz}, 1 \mathrm{H}) ;{ }^{13} \mathrm{C} \mathrm{NMR}\left(125 \mathrm{MHz}, \mathrm{CDCl}_{3}\right) \delta: 137.23,137.0,136.5$, 136.2, 130.8, 130.2, 129.6, 128.5, 128.1, 128.0, 79.0, 73.4, 49.0, 48.7, 47.8. $v_{\max }\left(\right.$ neat, $\left.\mathrm{cm}^{-1}\right): 3586$, $3029,1550,1453,1433,1378,1246,1060,1033$, 970. HRMS (ESI) $m / z$ calcd for $\mathrm{C}_{26} \mathrm{H}_{24} \mathrm{~N}_{2} \mathrm{NaO}_{5}$ $\left([\mathrm{M}+\mathrm{Na}]^{+}\right)$: 467.1577; found: 467.1573. Enantiomeric excess of $\mathbf{4 a}$ was determined by chiral stationary phase HPLC analysis using a ChiralPak IC column (90:10 hexanes/i-PrOH at 1.0 $\mathrm{mL} / \mathrm{min}, \lambda=220 \mathrm{~nm}$ ), minor enantiomer: $t_{R}=18.0$ min, major enantiomer: $t_{R}=50.0 \mathrm{~min}$.

$\left(1 ' R, 2^{\prime} S, 3 ' S, 4^{\prime} S\right)-4^{\prime}$ '-Methyl-3'-nitro-5'-[(R)-2-nitro-1-(p-tolyl)ethyl]-1',2',3',4'-tetrahydro$\left[1,1^{\prime}: 2 ', 1^{\prime \prime}-\right.$ terphenyl]-4'-ol (4b)

The crude material was purified by flash column chromatography using hexanes/EtOAc (93:7) as an eluent to get $\mathbf{4 b}$ as a white solid, $68.9 \mathrm{mg}$, 73\% yield, m.p. $130-132{ }^{\circ} \mathrm{C} ;{ }^{1} \mathrm{H} \mathrm{NMR}(500 \mathrm{MHz}$,

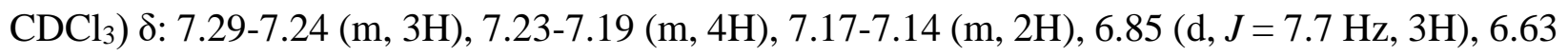
$(\mathrm{d}, J=7.4 \mathrm{~Hz}, 2 \mathrm{H}), 6.44(\mathrm{br}, 2 \mathrm{H}), 6.00(\mathrm{~d}, J=3.4 \mathrm{~Hz}, 1 \mathrm{H}), 5.21\left(\mathrm{dd}, J_{1}=12.2 \mathrm{~Hz}, J_{2}=9.7 \mathrm{~Hz}\right.$, $1 \mathrm{H}), 5.08\left(\mathrm{dd}, J_{1}=12.6 \mathrm{~Hz}, J_{2}=8.5 \mathrm{~Hz}, 1 \mathrm{H}\right), 4.91\left(\mathrm{dd}, J_{1}=12.3 \mathrm{~Hz}, J_{2}=7.0 \mathrm{~Hz}, 1 \mathrm{H}\right), 4.79(\mathrm{t}, J$ $=8.1 \mathrm{~Hz}, 1 \mathrm{H}), 4.59(\mathrm{~d}, J=7.3 \mathrm{~Hz}, 1 \mathrm{H}), 3.80-3.65(\mathrm{~m}, 2 \mathrm{H}), 2.55(\mathrm{~d}, J=7.6 \mathrm{~Hz}, 1 \mathrm{H}), 2.36(\mathrm{~s}, 3 \mathrm{H})$, 


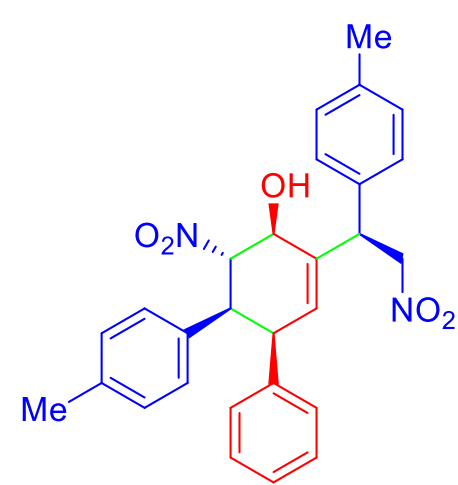

2.21 (s, 3H). ${ }^{13} \mathrm{C}$ NMR (125 MHz, $\left.\mathrm{CDCl}_{3}\right) \delta: 138.3,137.6,137.1$, 136.7, 134.2, 133.1, 130.7, 130.3, 130.3, 129.1, 128.4, 128.4, 127.9, 127.9, 90.8, 79.1, 73.5, 48.7, 47.6, 21.4, 21.4. $v_{\max }\left(\right.$ neat, $\left.\mathrm{cm}^{-1}\right): 3542$, 2922, 1548, 1514, 1452, 1374, 1265, 1050, 976, 899. HRMS (ESI) $m / z$ calcd for $\mathrm{C}_{28} \mathrm{H}_{27} \mathrm{~N}_{2} \mathrm{O}_{5}$ ([M-H]'): 471.1925; found: 471.1938. Enantiomeric excess of $\mathbf{4 b}$ was determined by chiral stationary phase HPLC analysis using a ChiralPak IB column (90:10 hexanes/ $i$-PrOH at $1.0 \mathrm{~mL} / \mathrm{min}, \lambda=220 \mathrm{~nm}$ ), minor enantiomer: $t_{R}=21.1 \mathrm{~min}$, major enantiomer: $t_{R}=22.9 \min$.

\section{(1'R,2'S,3'S,4'S)-4' '-Fluoro-5'-[(R)-1-(4-fluorophenyl)-2-nitroethyl]-3'-nitro-1',2',3',4'-} tetrahydro-[1,1':2',1'-terphenyl]-4'-ol (4c)

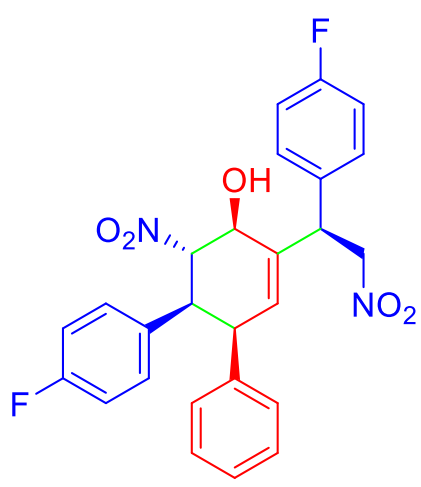

The crude material was purified by flash column chromatography using hexanes/EtOAc (91:9) as an eluent to get $\mathbf{4 c}$ as a white solid, $67.2 \mathrm{mg}$, $70 \%$ yield, m.p. $134-136{ }^{\circ} \mathrm{C} ;{ }^{1} \mathrm{H}$ NMR $\left(500 \mathrm{MHz}, \mathrm{CDCl}_{3}\right)$ 8: 7.36-7.33 (m, 2H), 7.24-7.21 (m, 1H), 7.18-7.15 (m, 2H), 7.08-7.05 (m, 2H), 6.76 (t, $J=8.5 \mathrm{~Hz}, 2 \mathrm{H}), 6.64-6.57(\mathrm{~m}, 3 \mathrm{H}), 6.52(\mathrm{br}, 2 \mathrm{H}), 5.98(\mathrm{~d}, J=3.3$ $\mathrm{Hz}, 1 \mathrm{H}), 5.18\left(\mathrm{dd}, J_{1}=12.4 \mathrm{~Hz}, J_{2}=9.5 \mathrm{~Hz}, 1 \mathrm{H}\right), 5.09-5.02(\mathrm{~m}, 1 \mathrm{H})$, $4.91\left(\mathrm{dd}, J_{1}=12.3 \mathrm{~Hz}, J_{2}=7.1 \mathrm{~Hz}, 1 \mathrm{H}\right), 4.78(\mathrm{t}, J=7.4 \mathrm{~Hz}, 1 \mathrm{H}), 4.62$ $\left(\mathrm{dd}, J_{1}=9.3, J_{2}=7.2 \mathrm{~Hz}, 1 \mathrm{H}\right), 3.77-3.73(\mathrm{~m}, 2 \mathrm{H}), 2.81(\mathrm{~d}, J=7.3 \mathrm{~Hz}, 1 \mathrm{H}) .{ }^{13} \mathrm{C}$ NMR $(126 \mathrm{MHz}$, $\left.\mathrm{CDCl}_{3}\right) \delta: 162.7\left(\mathrm{~d}, J_{\mathrm{C}-\mathrm{F}}=239.4 \mathrm{~Hz}\right), 162.5\left(\mathrm{~d}, J_{\mathrm{C}-\mathrm{F}}=239.4 \mathrm{~Hz}\right), 137.0,136.3,133.0,131.9,130.7$, $130.2,130.1\left(\mathrm{~d}, J_{\mathrm{C}-\mathrm{F}}=7.5 \mathrm{~Hz}\right), 129.8\left(\mathrm{~d}, J_{\mathrm{C}-\mathrm{F}}=7.5 \mathrm{~Hz}\right), 128.7,128.2,116.5\left(\mathrm{~d}, J_{\mathrm{C}-\mathrm{F}}=21.3 \mathrm{~Hz}\right)$, $115.4\left(\mathrm{~d}, J_{\mathrm{C}-\mathrm{F}}=21.3 \mathrm{~Hz}\right), 90.7,79.0,73.1,48.6,48.0,47.1 . v_{\max }\left(\right.$ neat, $\left.\mathrm{cm}^{-1}\right): 3526,2922,1603$, $1548,1507,1452,1347,1224,1160,1050$. HRMS (ESI) $\mathrm{m} / \mathrm{z}$ calcd for $\mathrm{C}_{26} \mathrm{H}_{21} \mathrm{~F}_{2} \mathrm{~N}_{2} \mathrm{O}_{5}\left([\mathrm{M}-\mathrm{H}]^{-}\right)$: 479.1424; found: 479.1430 . Enantiomeric excess of $\mathbf{4 c}$ was determined by chiral stationary phase HPLC analysis using a ChiralPak IC column (90:10 hexanes $/ i-\mathrm{PrOH}$ at $1.0 \mathrm{~mL} / \mathrm{min}, \lambda=220 \mathrm{~nm}$ ), minor enantiomer: $t_{R}=10.3$ min, major enantiomer: $t_{R}=31.8$ min.

$\left(1^{\prime} R, 2^{\prime} S, 3 ' S, 4^{\prime} S\right)-4^{\prime \prime}$-Chloro-5'-[(R)-1-(4-chlorophenyl)-2-nitroethyl]-3'-nitro-1',2',3',4' tetrahydro-[1,1':2',1"'-terphenyl]-4'-ol (4d) 


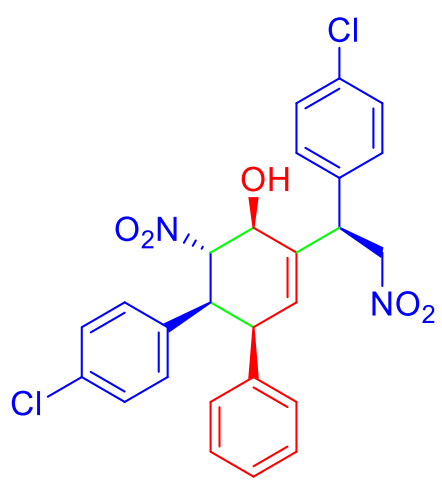

The crude material was purified by flash column chromatography using hexanes/EtOAc (92:8) as an eluent to get $\mathbf{4 d}$ as a white solid, $71.8 \mathrm{mg}, 70 \%$ yield, m.p. $140-142{ }^{\circ} \mathrm{C} ;{ }^{1} \mathrm{H}$ NMR $\left(500 \mathrm{MHz}, \mathrm{CDCl}_{3}\right)$ $\delta: ~ 7.37-7.34(\mathrm{~m}, 3 \mathrm{H}), 7.31-7.29(\mathrm{~m}, 2 \mathrm{H}), 7.23-7.20(\mathrm{~m}, 2 \mathrm{H})$, 7.19$7.16(\mathrm{~m}, 2 \mathrm{H}), 7.05(\mathrm{~d}, J=8.2 \mathrm{~Hz}, 2 \mathrm{H}), 6.60(\mathrm{~d}, J=7.5 \mathrm{~Hz}, 2 \mathrm{H}), 6.49$ (br, $2 \mathrm{H}), 5.97(\mathrm{~d}, J=3.2 \mathrm{~Hz}, 1 \mathrm{H}), 5.15\left(\mathrm{dd}, J_{1}=12.5 \mathrm{~Hz}, J_{2}=9.2 \mathrm{~Hz}\right.$, $1 \mathrm{H}), 5.06\left(\mathrm{dd}, J_{1}=12.1 \mathrm{~Hz}, J_{2}=8.3 \mathrm{~Hz}, 1 \mathrm{H}\right), 4.92-4.88(\mathrm{~m}, 2 \mathrm{H}), 4.76$ $(\mathrm{t}, J=7.6 \mathrm{~Hz}, 1 \mathrm{H}), 4.61\left(\mathrm{dd}, J_{1}=9.1 \mathrm{~Hz}, J_{2}=7.3 \mathrm{~Hz}, 1 \mathrm{H}\right), 3.79-3.69(\mathrm{~m}, 2 \mathrm{H}), 2.92(\mathrm{~d}, J=7.1 \mathrm{~Hz}$, 1H). ${ }^{13} \mathrm{C}$ NMR (125 MHz, $\left.\mathrm{CDCl}_{3}\right) \delta: 136.8,136.1,135.8,134.6,134.3,134.0,130.7,130.2,129.8$, 129.7, 129.5, 128.7, 128.2, 90.5, 78.7, 73.0, 48.4, 47.9, 47.2. $v_{\max }$ (neat, $\mathrm{cm}^{-1}$ ): 3501, 2918, 1548, 1490, 1452, 1373, 1091, 1051, 1013, 972. HRMS (ESI) $\mathrm{m} / z$ calcd for $\mathrm{C}_{26} \mathrm{H}_{21} \mathrm{Cl}_{2} \mathrm{~N}_{2} \mathrm{O}_{5}\left([\mathrm{M}-\mathrm{H}]^{-}\right)$: 511.0833; found: 511.0830. Enantiomeric excess of $\mathbf{4 d}$ was determined by chiral stationary phase HPLC analysis using a ChiralPak IC column (90:10 hexanes $/ i-\mathrm{PrOH}$ at $1.0 \mathrm{~mL} / \mathrm{min}, \lambda=220 \mathrm{~nm}$ ), minor enantiomer: $t_{R}=11.0$ min, major enantiomer: $t_{R}=32.5 \mathrm{~min}$.

(1'R,2'S,3' $\left.S, 4^{\prime} S\right)$-4' '-Bromo-5'-[(R)-1-(4-bromophenyl)-2-nitroethyl]-3'-nitro-1',2',3',4'tetrahydro-[1,1':2',1"'-terphenyl]-4'-ol (4e)

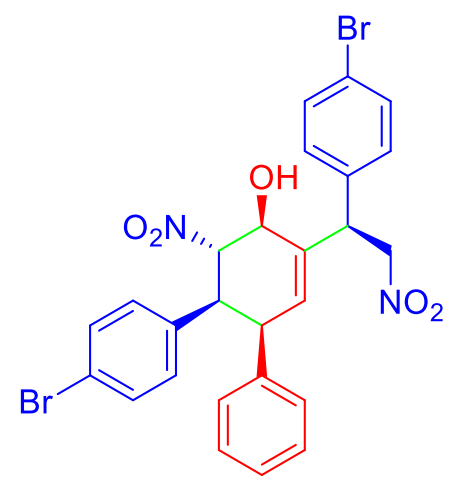

The crude material was purified by flash column chromatography using hexanes/EtOAc (91:9) as an eluent to get $\mathbf{4 e}$ as a light green solid, $90.3 \mathrm{mg}$, 75\% yield, m.p. $150-152{ }^{\circ} \mathrm{C} ;{ }^{1} \mathrm{H}$ NMR $(500 \mathrm{MHz}$, $\left.\mathrm{CDCl}_{3}\right) \delta: 7.51(\mathrm{~d}, J=8.4 \mathrm{~Hz}, 2 \mathrm{H}), 7.26-7.16(\mathrm{~m}, 9 \mathrm{H}), 6.60(\mathrm{~d}, J=7.2$ $\mathrm{Hz}, 2 \mathrm{H}), 6.43(\mathrm{br}, 2 \mathrm{H}), 5.98(\mathrm{~d}, J=3.5 \mathrm{~Hz}, 1 \mathrm{H}), 5.16\left(\mathrm{dd}, J_{1}=12.5\right.$ $\left.\mathrm{Hz}, J_{2}=9.3 \mathrm{~Hz}, 1 \mathrm{H}\right), 5.05\left(\mathrm{dd}, J_{1}=12.6 \mathrm{~Hz}, J_{2}=8.4 \mathrm{~Hz}, 1 \mathrm{H}\right), 4.90$ $\left(\mathrm{dd}, J_{1}=12.5 \mathrm{~Hz}, J_{2}=7.2 \mathrm{~Hz}, 1 \mathrm{H}\right), 4.76(\mathrm{t}, J=7.6 \mathrm{~Hz}, 1 \mathrm{H}), 4.63-$ $4.56(\mathrm{~m}, 1 \mathrm{H}), 3.80-3.67(\mathrm{~m}, 2 \mathrm{H}), 2.75(\mathrm{~d}, J=7.2 \mathrm{~Hz}, 1 \mathrm{H}) .{ }^{13} \mathrm{C} \mathrm{NMR}\left(125 \mathrm{MHz}, \mathrm{CDCl}_{3}\right) \delta: 136.7$, 136.3, 136.0, 135.1, 132.7, 131.7, 130.8, 130.2, 129.8, 128.8, 128.3, 122.5, 122.2, 90.4, 78.6, 73.1, 48.3, 48.1, 47.3. $v_{\max }\left(\right.$ neat, $\left.\mathrm{cm}^{-1}\right): 3500,2918,1547,1487,1452,1432,1373,1099,1073,1009$, 904. HRMS (ESI) $\mathrm{m} / z$ calcd for $\mathrm{C}_{26} \mathrm{H}_{21} \mathrm{Br}_{2} \mathrm{~N}_{2} \mathrm{O}_{5}$ ([M-H] $]^{-}$): 598.9823; found: 598.9813. Enantiomeric excess of $\mathbf{4 e}$ was determined by chiral stationary phase HPLC analysis using a 
ChiralPak IC column (92.5:7.5 hexanes $/ i-\mathrm{PrOH}$ at $1.0 \mathrm{~mL} / \mathrm{min}, \lambda=220 \mathrm{~nm})$, minor enantiomer: $t_{\mathrm{R}}$ $=15.1 \mathrm{~min}$, major enantiomer: $\mathrm{t}_{\mathrm{R}}=53.3 \mathrm{~min}$.

(1'R,2'S,3'S,4'S)-2' '-Bromo-5'-[(S)-1-(2-bromophenyl)-2-nitroethyl]-3'-nitro-1',2',3',4'tetrahydro-[1,1':2',1'-terphenyl]-4'-ol (4f)

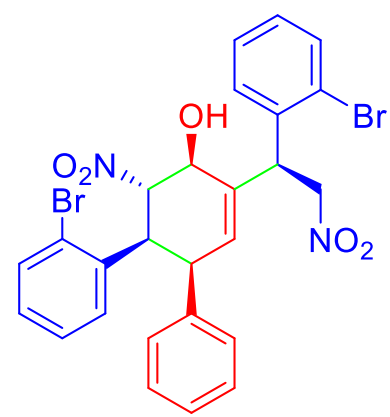

The crude material was purified by flash column chromatography using hexanes/EtOAc (93:7) as an eluent to get $\mathbf{4 f}$ as a brown solid, $78.3 \mathrm{mg}$, $65 \%$ yield, m.p. $125-127{ }^{\circ} \mathrm{C} ;{ }^{1} \mathrm{H}$ NMR $\left(500 \mathrm{MHz}, \mathrm{CDCl}_{3}\right) \delta: 7.66(\mathrm{~d}, J=$ $6.7 \mathrm{~Hz}, 1 \mathrm{H}), 7.56\left(\mathrm{dd}, J_{1}=7.9 \mathrm{~Hz}, J_{2}=1.3 \mathrm{~Hz}, 1 \mathrm{H}\right), 7.43-7.41(\mathrm{~m}, 1 \mathrm{H})$, 7.25-7.23 (m, 1H), $7.16(\mathrm{t}, J=7.6 \mathrm{~Hz}, 2 \mathrm{H}), 7.11(\mathrm{t}, J=7.1 \mathrm{~Hz}, 2 \mathrm{H}), 6.98$ $\left(\mathrm{td}, J_{1}=7.7 \mathrm{~Hz}, J_{2}=1.6 \mathrm{~Hz}, 1 \mathrm{H}\right), 6.79\left(\mathrm{td}, J_{1}=7.6 \mathrm{~Hz}, J_{2}=1.3 \mathrm{~Hz}, 1 \mathrm{H}\right)$, 6.61-6.68 (m, 2H), $6.04\left(\mathrm{dd}, J_{1}=7.9 \mathrm{~Hz}, J_{2}=1.6 \mathrm{~Hz}, 1 \mathrm{H}\right), 5.93(\mathrm{~d}, J=3.7 \mathrm{~Hz}, 1 \mathrm{H}), 5.20-5.13(\mathrm{~m}$, $3 \mathrm{H}), 4.99-4.94(\mathrm{~m}, 1 \mathrm{H}), 4.76\left(\mathrm{dd}, J_{1}=8.5 \mathrm{~Hz}, 6.9 J_{2}=\mathrm{Hz}, 1 \mathrm{H}\right), 4.36\left(\mathrm{dd}, J_{1}=12.9 \mathrm{~Hz}, J_{2}=6.1\right.$ $\mathrm{Hz}, 1 \mathrm{H}), 4.03(\mathrm{t}, J=4.9 \mathrm{~Hz}, 1 \mathrm{H}), 2.90(\mathrm{~d}, J=7.1 \mathrm{~Hz}, 1 \mathrm{H}) \cdot{ }^{13} \mathrm{C} \mathrm{NMR}\left(125 \mathrm{MHz}, \mathrm{CDCl}_{3}\right) \delta: 136.7$, 135.9, 135.3, 134.7, 134.5, 133.4, 132.1, 129.9, 129.9, 129.3, 128.5, 128.4, 128.3, 128.3, 128.0, 127.2, 125.8, 125.0, 90.2, 77.8, 73.1, 47.4, 45.9, 45.1. $v_{\max }\left(\right.$ neat, $\mathrm{cm}^{-1}$ ): 3503, 2919, 2850, 1548, $1489,1471,1452,1373,1263,1062,1021,973$. HRMS (ESI) $m / z$ calcd for $\mathrm{C}_{26} \mathrm{H}_{21} \mathrm{Br}_{2} \mathrm{~N}_{2} \mathrm{O}_{5}$ ([M$\mathrm{H}^{-}$): 598.9823; found: 598.9822. Enantiomeric excess of $\mathbf{4 f}$ was determined by chiral stationary phase HPLC analysis using a ChiralPak IC column (90:10 hexanes $/ i-\mathrm{PrOH}$ at $1.0 \mathrm{~mL} / \mathrm{min}, \lambda=220$ $\mathrm{nm}$ ), minor enantiomer: $\mathrm{t}_{\mathrm{R}}=19.6$ min, major enantiomer: $\mathrm{t}_{\mathrm{R}}=40.4 \mathrm{~min}$.

(1'R,2'S,3' $S, 4$ ' $S)$-3''-Bromo-5'-[(R)-1-(3-bromophenyl)-2-nitroethyl]-3'-nitro-1',2',3',4'tetrahydro-[1,1':2',1"'-terphenyl]-4'-ol (4g)

The crude material was purified by flash column chromatography using hexanes/EtOAc (95:5) as an eluent to get $\mathbf{4 g}$ as a light green solid, $86.7 \mathrm{mg}, 68 \%$ yield, m.p. $125-127{ }^{\circ} \mathrm{C} ;{ }^{1} \mathrm{H}$ NMR $(500$ $\left.\mathrm{MHz}, \mathrm{CDCl}_{3}\right)$ d: $7.52(\mathrm{~s}, 1 \mathrm{H}), 7.48-7.45(\mathrm{~m}, 2 \mathrm{H}), 7.33-7.18(\mathrm{~m}, 8 \mathrm{H}), 6.91(\mathrm{t}, J=7.9 \mathrm{~Hz}, 1 \mathrm{H}), 6.80$ (br, $1 \mathrm{H}), 6.61(\mathrm{~d}, J=6.8 \mathrm{~Hz}, 2 \mathrm{H}), 6.41(\mathrm{br}, 1 \mathrm{H}), 6.00(\mathrm{~d}, J=4.1 \mathrm{~Hz}, 1 \mathrm{H}), 5.15\left(\mathrm{dd}, J_{1}=12.6 \mathrm{~Hz}\right.$, 


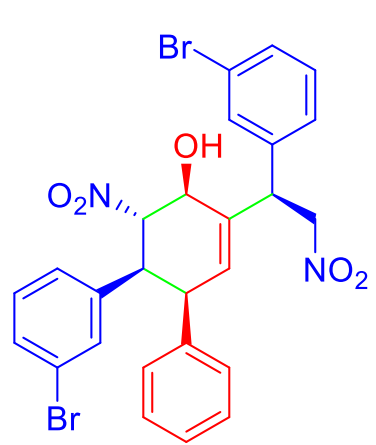

$\left.J_{2}=9.4 \mathrm{~Hz}, 1 \mathrm{H}\right), 5.06\left(\mathrm{dd}, J_{1}=12.3 \mathrm{~Hz}, J_{2}=8.4 \mathrm{~Hz}, 1 \mathrm{H}\right), 4.93-4.87(\mathrm{~m}$, $1 \mathrm{H}), 4.77(\mathrm{t}, J=10.0 \mathrm{~Hz}, 1 \mathrm{H}), 4.60\left(\mathrm{dd}, J_{1}=9.5 \mathrm{~Hz}, J_{2}=6.9 \mathrm{~Hz}, 1 \mathrm{H}\right)$, 3.80-3.73 (m, 2H), $2.81(\mathrm{~d}, J=7.1 \mathrm{~Hz}, 1 \mathrm{H}) .{ }^{13} \mathrm{C} \mathrm{NMR}\left(125 \mathrm{MHz}, \mathrm{CDCl}_{3}\right)$ $\delta: 139.6,138.4,136.6,136.0,132.0,131.6,131.3,131.2,131.1,130.8$, $130.2,130.1,129.9,128.7,128.3,126.7,123.6,122.5,90.2,78.6,73.1$, 48.4, 48.1, 47.3. $v_{\max }\left(\right.$ neat, $\left.\mathrm{cm}^{-1}\right): 3501,2921,2851,1594,1547,1489$, 1474, 1452, 1373, 1260, 1199, 996. HRMS (ESI) $\mathrm{m} / \mathrm{z}$ calcd for

$\mathrm{C}_{26} \mathrm{H}_{21} \mathrm{Br}_{2} \mathrm{~N}_{2} \mathrm{O}_{5}\left([\mathrm{M}-\mathrm{H}]^{-}\right)$: 598.9823; found: 598.9833. Enantiomeric excess of $\mathbf{4 g}$ was determined by chiral stationary phase HPLC analysis using a ChiralPak IC column (95:5 hexanes/i-PrOH at $1.0 \mathrm{~mL} / \mathrm{min}, \lambda=220 \mathrm{~nm}$ ), minor enantiomer: $t_{R}=32.3 \mathrm{~min}$, major enantiomer: $t_{R}=85.3 \mathrm{~min}$.

\section{$(1 R, 2 R, 3 S, 4 S)-3-N i t r o-5-[(R)-2-n i t r o-1-(t h i o p h e n-2-y l) e t h y l]-2-(t h i o p h e n-2-y l)-1,2,3,4-$}

\section{tetrahydro-[1,1'-biphenyl]-4-ol (4h)}

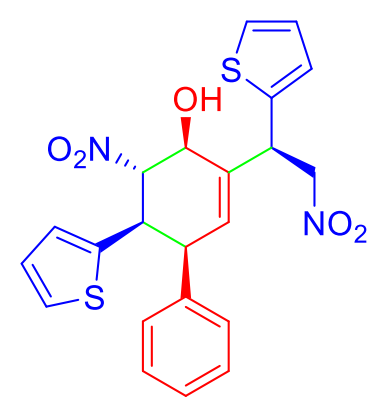

The crude material was purified by flash column chromatography using hexanes/EtOAc (90:10) as an eluent to get $\mathbf{4 h}$ as a white solid, $62.1 \mathrm{mg}$, $68 \%$ yield, m.p. $140-142{ }^{\circ} \mathrm{C} ;{ }^{1} \mathrm{H}$ NMR (500 MHz, $\left.\mathrm{CDCl}_{3}\right)$ ): 7.30-7.28 (m, $1 \mathrm{H}), 7.25-7.17(\mathrm{~m}, 3 \mathrm{H}), 7.08-6.98(\mathrm{~m}, 3 \mathrm{H}), 6.75(\mathrm{~d}, J=6.7 \mathrm{~Hz}, 2 \mathrm{H}), 6.69-$ $6.68(\mathrm{~m}, 1 \mathrm{H}), 6.10(\mathrm{~d}, J=3.5 \mathrm{~Hz}, 1 \mathrm{H}), 6.05(\mathrm{~d}, J=4.6 \mathrm{~Hz}, 1 \mathrm{H}), 5.19$ (dd, $\left.J_{1}=12.2 \mathrm{~Hz}, J_{2}=9.1 \mathrm{~Hz}, 1 \mathrm{H}\right), 5.05\left(\mathrm{dd}, J_{1}=12.6 \mathrm{~Hz}, J_{2}=8.5 \mathrm{~Hz}, 1 \mathrm{H}\right)$, $4.96\left(\mathrm{dd}, J_{1}=12.2 \mathrm{~Hz}, J_{2}=6.9 \mathrm{~Hz}, 1 \mathrm{H}\right), 4.92-4.89(\mathrm{~m}, 2 \mathrm{H}), 4.08\left(\mathrm{dd}, J_{1}=12.7 \mathrm{~Hz}, J_{2}=6.1 \mathrm{~Hz}\right.$, 1H), $3.86(\mathrm{t}, J=5.4 \mathrm{~Hz}, 1 \mathrm{H}), 2.74(\mathrm{~d}, J=7.3 \mathrm{~Hz}, 1 \mathrm{H}) .{ }^{13} \mathrm{C} \mathrm{NMR}\left(125 \mathrm{MHz}, \mathrm{CDCl}_{3}\right) \delta: 139.9$, 138.1, 136.6, 136.6, 130.9, 130.1, 129.9, 128.7, 128.6, 128.2, 127.8, 127.0, 127.0, 126.3, 126.1, 125.9, 124.9, 91.5, 79.5, 72.8, 48.9, 44.3, 42.9. $v_{\max }\left(\right.$ neat, $\left.\mathrm{cm}^{-1}\right): 3581,2918,2849,1550,1490$, 1430, 1379, 1330, 1246, 1201, 1049, 967. HRMS (ESI) $\mathrm{m} / z$ calcd for $\mathrm{C}_{22} \mathrm{H}_{19} \mathrm{~N}_{2} \mathrm{O}_{5} \mathrm{~S}_{2}\left([\mathrm{M}-\mathrm{H}]^{-}\right)$: 455.0741; found: 455.0750 . Enantiomeric excess of $\mathbf{4 h}$ was determined by chiral stationary phase HPLC analysis using a ChiralPak IC column (90:10 hexanes $/ i-\mathrm{PrOH}$ at $1.0 \mathrm{~mL} / \mathrm{min}, \lambda=220 \mathrm{~nm}$ ), minor enantiomer: $t_{R}=19.4$ min, major enantiomer: $t_{R}=46.2 \mathrm{~min}$. 


\section{$(1 ' R, 2 ' S, 3$ ' $S, 4$ ' $S)$-4-Methoxy-3'-nitro-5'-[(R)-2-nitro-1-phenylethyl]-1',2',3',4'-tetrahydro-}

\section{[1,1':2',1''-terphenyl]-4' -ol (4i)}

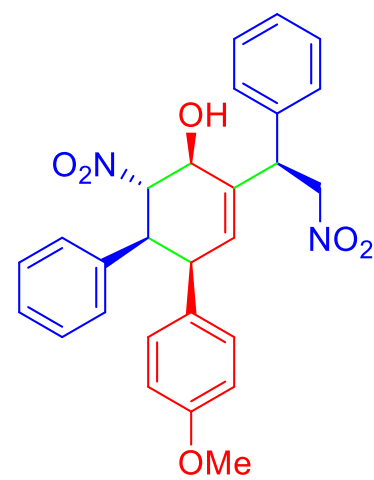

The crude material was purified by flash column chromatography using hexanes/EtOAc (90:10) as an eluent to get $4 \mathbf{i}$ as a white solid, $64.5 \mathrm{mg}$, $68 \%$ yield, m.p. $120-122{ }^{\circ} \mathrm{C} ;{ }^{1} \mathrm{H}$ NMR (500 $\left.\mathrm{MHz} \mathrm{CDCl}_{3}\right)$ ): 7.48-7.33 (m, 5H), 7.15-7.06 (m, 3H), $6.68(\mathrm{~d}, J=8.8 \mathrm{~Hz}, 2 \mathrm{H}), 6.59(\mathrm{br}, 2 \mathrm{H}), 6.50$ $(\mathrm{d}, J=8.2 \mathrm{~Hz}, 2 \mathrm{H}), 6.01(\mathrm{~d}, J=3.2 \mathrm{~Hz}, 1 \mathrm{H}), 5.25\left(\mathrm{dd}, J_{1}=12.2 \mathrm{~Hz}, J_{2}=\right.$ $9.8 \mathrm{~Hz}, 1 \mathrm{H}), 5.08\left(\mathrm{dd}, J_{1}=12.0 \mathrm{~Hz}, J_{2}=8.5 \mathrm{~Hz}, 1 \mathrm{H}\right), 4.94\left(\mathrm{dd}, J_{1}=12.2\right.$ $\left.\mathrm{Hz}, J_{2}=6.8 \mathrm{~Hz}, 1 \mathrm{H}\right), 4.79-4.76(\mathrm{~m}, 1 \mathrm{H}), 4.61\left(\mathrm{dd}, J_{1}=9.7 \mathrm{~Hz}, J_{2}=6.9\right.$ $\mathrm{Hz}, 1 \mathrm{H}), 3.76(\mathrm{~s}, 3 \mathrm{H}), 3.73-3.70(\mathrm{~m}, 2 \mathrm{H}), 2.62(\mathrm{~d}, J=7.5 \mathrm{~Hz}, 1 \mathrm{H}) .{ }^{13} \mathrm{C} \mathrm{NMR}\left(126 \mathrm{MHz}, \mathrm{CDCl}_{3}\right) \delta$ : 159.4, 137.3, 136.6, 136.4, 131.3, 131.3, 129.6, 128.5, 128.5, 128.4, 128.0, 127.9, 113.8, 90.7, 79.1, 77.6, 73.4, 55.6, 49.1, 47.9. $v_{\max }\left(\right.$ neat, $\left.\mathrm{cm}^{-1}\right): 3501,2920,2850,1606,1547,1508,1495$, 1454, 1374, 1333, 1247, 1176, 1112, 1030. HRMS (ESI) $\mathrm{m} / \mathrm{z}$ calcd for $\mathrm{C}_{27} \mathrm{H}_{25} \mathrm{~N}_{2} \mathrm{O}_{6}\left([\mathrm{M}-\mathrm{H}]^{-}\right)$: 473.1718; found: 473.1720. Enantiomeric excess of $\mathbf{4 i}$ was determined by chiral stationary phase HPLC analysis using a ChiralPak IC column (90:10 hexanes $/ i-\mathrm{PrOH}$ at $1.0 \mathrm{~mL} / \mathrm{min}, \lambda=220 \mathrm{~nm}$ ), minor enantiomer: $t_{R}=23.4$ min, major enantiomer: $t_{R}=71.7$ min.

(1'R,2' $S, 3$ ' $S, 4$ ' $S)$-4-Fluoro-3'-nitro-5'-[(R)-2-nitro-1-phenylethyl]-1',2',3',4'-tetrahydro$\left[1,1^{\prime}: 2^{\prime}, 1^{\prime \prime}\right.$-terphenyl]-4'-ol (4j)

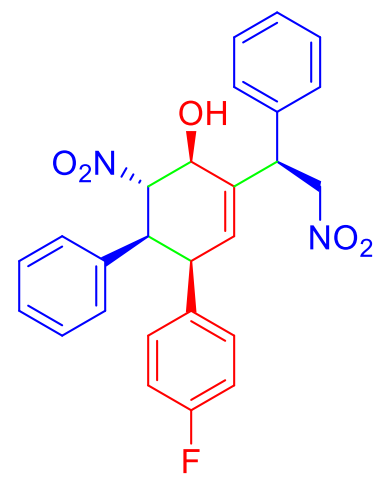

The crude material was purified by flash column chromatography using hexanes/EtOAc (95:5) as an eluent to get $\mathbf{4} \mathbf{j}$ as a white solid, $58.3 \mathrm{mg}, 63 \%$ yield, m.p. $130-132{ }^{\circ} \mathrm{C} ;{ }^{1} \mathrm{H} \mathrm{NMR}\left(500 \mathrm{MHz} \mathrm{CDCl}_{3}\right) \delta$ : 7.37-7.32 (m, 6H), $7.14(\mathrm{~d}, J=7.1 \mathrm{~Hz}, 1 \mathrm{H}), 7.09(\mathrm{t}, J=7.3 \mathrm{~Hz}, 2 \mathrm{H}), 6.83(\mathrm{t}, J=8.5 \mathrm{~Hz}, 2 \mathrm{H})$, 6.67-6.57 (m, 2H), $6.55\left(\mathrm{dd}, J_{1}=8.3 \mathrm{~Hz}, J_{2}=5.3 \mathrm{~Hz}, 2 \mathrm{H}\right), 5.99(\mathrm{q}, J=2.0$ $\mathrm{Hz}, 1 \mathrm{H}), 5.26\left(\mathrm{dd}, J_{1}=12.2 \mathrm{~Hz}, J_{2}=9.8 \mathrm{~Hz}, 1 \mathrm{H}\right), 5.06\left(\mathrm{dd}, J_{1}=12.1 \mathrm{~Hz}\right.$, $\left.J_{2}=8.5 \mathrm{~Hz}, 1 \mathrm{H}\right), 4.93\left(\mathrm{dd}, J_{1}=12.2 \mathrm{~Hz}, J_{2}=6.7 \mathrm{~Hz}, 1 \mathrm{H}\right), 4.77(\mathrm{t}, J=7.8$ $\mathrm{Hz}, 1 \mathrm{H}), 4.62\left(\mathrm{dd}, J_{1}=9.7 \mathrm{~Hz}, J_{2}=6.7 \mathrm{~Hz}, 1 \mathrm{H}\right), 3.78-3.74(\mathrm{~m}, 2 \mathrm{H}), 2.79(\mathrm{~d}, J=7.4 \mathrm{~Hz}, 1 \mathrm{H}) .{ }^{13} \mathrm{C}$ NMR $\left(126 \mathrm{MHz}, \mathrm{CDCl}_{3}\right) \delta: 162.5\left(\mathrm{~d}, J_{\mathrm{C}-\mathrm{F}}=248.2 \mathrm{~Hz}\right), 137.3,137.2,136.1,132.3\left(\mathrm{~d}, J_{C-F}=2.5\right.$ $\mathrm{Hz}), 131.6\left(\mathrm{~d}, J_{C-F}=7.5 \mathrm{~Hz}\right), 130.7,129.6,128.6,128.5,128.3,128.3,128.1,128.0,115.3\left(\mathrm{~d}, J_{C-F}\right.$ 
$=21.3 \mathrm{~Hz}), 79.0,73.2,49.0,47.9,47.6 . v_{\max }\left(\right.$ neat, $\left.\mathrm{cm}^{-1}\right): 3509,2918,1602,1546,1506,1454$, 1374, 1331, 1275, 1222, 1158, 1059. HRMS (ESI) $m / z$ calcd for $\mathrm{C}_{26} \mathrm{H}_{22} \mathrm{FN}_{2} \mathrm{O}_{5}$ ([M-H] $]^{-}$: 461.1518 ; found: 461.1519. Enantiomeric excess of $\mathbf{4} \mathbf{j}$ was determined by chiral stationary phase HPLC analysis using a ChiralPak IC column (90:10 hexanes $/ i-\mathrm{PrOH}$ at $1.0 \mathrm{~mL} / \mathrm{min}, \lambda=220 \mathrm{~nm})$, minor enantiomer: $t_{R}=15.0$ min, major enantiomer: $t_{R}=44.0 \mathrm{~min}$.

(1'R,2'S,3' $S, 4$ 'S)-4-Chloro-3'-nitro-5'-[(R)-2-nitro-1-phenylethyl]-1',2',3',4'-tetrahydro$\left[1,1^{\prime}: 2^{\prime}, 1^{\prime \prime}\right.$-terphenyl]-4'-ol (4k)

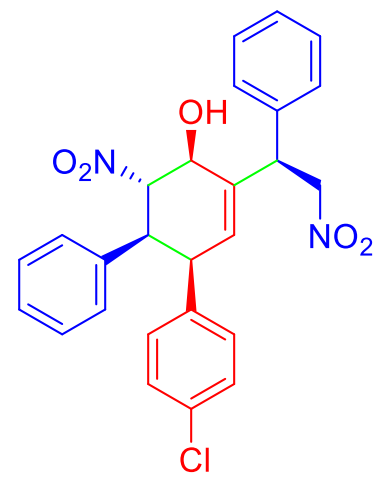

The crude material was purified by flash column chromatography using hexanes/EtOAc (95:5) as an eluent to get $\mathbf{4 k}$ as a pale yellow solid, 58.3 mg, $61 \%$ yield, m.p. $135-137{ }^{\circ} \mathrm{C} ;{ }^{1} \mathrm{H}$ NMR $\left(500 \mathrm{MHz}, \mathrm{CDCl}_{3}\right) \delta: 7.38-$ $7.32(\mathrm{~m}, 6 \mathrm{H}), 7.15-7.10(\mathrm{~m}, 6 \mathrm{H}), 6.60(\mathrm{br}, 2 \mathrm{H}), 6.51(\mathrm{~d}, J=8.1 \mathrm{~Hz}, 2 \mathrm{H})$, $5.96(\mathrm{~d}, J=2.9 \mathrm{~Hz}, 1 \mathrm{H}), 5.26\left(\mathrm{dd}, J_{1}=12.2 \mathrm{~Hz}, J_{2}=9.8 \mathrm{~Hz}, 1 \mathrm{H}\right), 5.05$ $\left(\mathrm{dd}, J_{1}=12.3 \mathrm{~Hz}, J_{2}=8.4 \mathrm{~Hz}, 1 \mathrm{H}\right), 4.92\left(\mathrm{dd}, J_{1}=12.2 \mathrm{~Hz}, J_{2}=6.7 \mathrm{~Hz}\right.$, $1 \mathrm{H}), 4.77(\mathrm{t}, J=7.6 \mathrm{~Hz}, 1 \mathrm{H}), 4.61\left(\mathrm{dd}, J_{1}=9.9 \mathrm{~Hz}, J_{2}=6.7 \mathrm{~Hz}, 1 \mathrm{H}\right), 3.80-$

$3.74(\mathrm{~m}, 2 \mathrm{H}), 2.80(\mathrm{~d}, J=7.4 \mathrm{~Hz}, 1 \mathrm{H}) .{ }^{13} \mathrm{C}$ NMR $\left(125 \mathrm{MHz}, \mathrm{CDCl}_{3}\right) \delta: 137.6,137.1,136.0,135.1$, 134.0, 131.4, 130.4, 129.6, 128.7, 128.6, 128.5, 128.3, 128.3, 128.1, 128.0, 90.4, 79.0, 73.2, 48.9, 48.0, 47.5. $v_{\max }\left(\right.$ neat, $\left.\mathrm{cm}^{-1}\right): 3523,3029,1547,1489,1454,1374,1274,1091,1013$. HRMS (ESI) $\mathrm{m} / \mathrm{z}$ calcd for $\mathrm{C}_{26} \mathrm{H}_{23} \mathrm{ClN}_{2} \mathrm{O}_{5}\left([\mathrm{M}+\mathrm{Na}]^{+}\right): 501.1188$; found: 501.1172. Enantiomeric excess of $\mathbf{4 k}$ was determined by chiral stationary phase HPLC analysis using a ChiralPak IC column (90:10 hexanes $/ i-\mathrm{PrOH}$ at $1.0 \mathrm{~mL} / \mathrm{min}, \lambda=220 \mathrm{~nm}$ ), minor enantiomer: $\mathrm{t}_{\mathrm{R}}=15.7$ min, major enantiomer: $\mathrm{t}_{\mathrm{R}}=49.3 \mathrm{~min}$.

$\left(1 ' R, 2^{\prime} S, 3\right.$ ' $S, 4$ ' $\left.S\right)$-4-Bromo-3'-nitro-5'-[(R)-2-nitro-1-phenylethyl]-1',2',3',4'-tetrahydro[1,1':2',1''-terphenyl]-4'-ol (4l) 


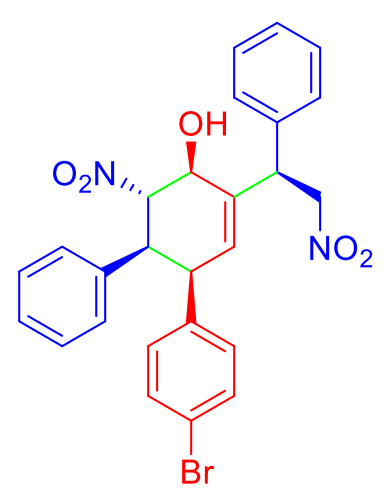

The crude material was purified by flash column chromatography using hexanes/EtOAc (95:5) as an eluent to get $\mathbf{4 I}$ as a pale yellow solid, 64.9 mg, $62 \%$ yield, m.p. $140-142{ }^{\circ} \mathrm{C} ;{ }^{1} \mathrm{H}$ NMR $\left(500 \mathrm{MHz}, \mathrm{CDCl}_{3}\right) \delta: 7.41-$ $7.30(\mathrm{~m}, 7 \mathrm{H}), 7.27-7.25(\mathrm{~m}, 4 \mathrm{H}), 7.18-7.08(\mathrm{~m}, 4 \mathrm{H}), 6.60(\mathrm{br}, 2 \mathrm{H}), 6.45$ $(\mathrm{d}, J=8.0 \mathrm{~Hz}, 2 \mathrm{H}), 5.96(\mathrm{~d}, J=3.1 \mathrm{~Hz}, 1 \mathrm{H}), 5.26\left(\mathrm{dd}, J_{1}=12.2 \mathrm{~Hz}, J_{2}=\right.$ $9.9 \mathrm{~Hz}, 1 \mathrm{H}), 5.05\left(\mathrm{dd}, J_{1}=12.1 \mathrm{~Hz}, J_{2}=8.4 \mathrm{~Hz}, 1 \mathrm{H}\right), 4.92\left(\mathrm{dd}, J_{1}=12.2\right.$ $\left.\mathrm{Hz}, J_{2}=6.7 \mathrm{~Hz}, 1 \mathrm{H}\right), 4.77(\mathrm{t}, J=7.9 \mathrm{~Hz}, 1 \mathrm{H}), 4.61\left(\mathrm{dd}, J_{1}=9.9 \mathrm{~Hz}, J_{2}=\right.$ $6.7 \mathrm{~Hz}, 1 \mathrm{H}), 3.79-3.73(\mathrm{~m}, 2 \mathrm{H}), 2.75(\mathrm{~d}, J=7.4 \mathrm{~Hz}, 1 \mathrm{H}) .{ }^{13} \mathrm{C} \mathrm{NMR}\left(125 \mathrm{MHz}, \mathrm{CDCl}_{3}\right) \delta: 137.62$, 137.10, 135.96, 135.65, 131.78, 131.54, 130.35, 129.60, 128.67, 128.49, 128.32, 128.29, 128.15, 127.98, 122.14, 90.34, 78.96, 73.21, 48.91, 48.08, 47.44. $v_{\max }\left(\right.$ neat, $\left.\mathrm{cm}^{-1}\right): 2522,2360,2340,2159$, 2016, 1868, 1699, 1683, 1652, 1558, 1540, 1456, 1418, 1072. HRMS (ESI) $\mathrm{m} / \mathrm{z}$ calcd for $\mathrm{C}_{26} \mathrm{H}_{23} \mathrm{BrN}_{2} \mathrm{O}_{5} \mathrm{Na}\left([\mathrm{M}+\mathrm{Na}]^{+}\right)$: 545.0683; found: 545.0663. Enantiomeric excess of $4 \mathbf{l}$ was determined by chiral stationary phase HPLC analysis using a ChiralPak IC column (90:10 hexanes $/ i-\mathrm{PrOH}$ at $1.0 \mathrm{~mL} / \mathrm{min}, \lambda=220 \mathrm{~nm}$ ), minor enantiomer: $\mathrm{t}_{\mathrm{R}}=16.7 \mathrm{~min}$, major enantiomer: $\mathrm{t}_{\mathrm{R}}=50.9 \mathrm{~min}$.

\section{(1'R,2'S,3' $S, 4$ ' $S)-4$ ''-Bromo-5'-[(R)-1-(4-bromophenyl)-2-nitroethyl]-4-methoxy-3'-nitro-} 1',2',3',4'-tetrahydro-[1,1':2',1''-terphenyl]-4'-ol (4m)

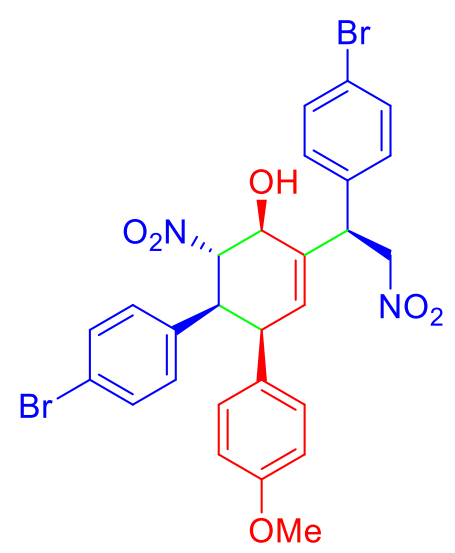

The crude material was purified by flash column chromatography using hexanes/EtOAc (95:5) as an eluent to get $\mathbf{4 m}$ as a white solid, $82.2 \mathrm{mg}, 65 \%$ yield, m.p. $135-137{ }^{\circ} \mathrm{C} ;{ }^{1} \mathrm{H}$ NMR $\left(500 \mathrm{MHz}, \mathrm{CDCl}_{3}\right) \delta$ : $7.51(\mathrm{~d}, J=8.4 \mathrm{~Hz}, 2 \mathrm{H}), 7.25-7.21(\mathrm{~m}, 6 \mathrm{H}), 6.71(\mathrm{~d}, J=8.8 \mathrm{~Hz}, 2 \mathrm{H})$, $6.50(\mathrm{~d}, J=8.2 \mathrm{~Hz}, 2 \mathrm{H}), 6.45(\mathrm{br}, 2 \mathrm{H}), 5.97(\mathrm{~d}, J=3.9 \mathrm{~Hz}, 1 \mathrm{H}), 5.18$ $\left(\mathrm{dd}, J_{1}=12.4 \mathrm{~Hz}, J_{2}=9.5 \mathrm{~Hz}, 1 \mathrm{H}\right), 5.02\left(\mathrm{dd}, J_{1}=12.5 \mathrm{~Hz}, J_{2}=8.4\right.$ $\mathrm{Hz}, 1 \mathrm{H}), 4.90\left(\mathrm{dd}, J_{1}=12.4 \mathrm{~Hz}, J_{2}=7.1 \mathrm{~Hz}, 1 \mathrm{H}\right), 4.74(\mathrm{t}, J=6.9 \mathrm{~Hz}$, $1 \mathrm{H}), 4.57\left(\mathrm{dd}, J_{1}=9.4 \mathrm{~Hz}, J_{2}=7.1 \mathrm{~Hz}, 1 \mathrm{H}\right), 3.78(\mathrm{~s}, 3 \mathrm{H}), 3.72-3.65$ (m, 2H), $2.75(\mathrm{~d}, J=7.2 \mathrm{~Hz}, 1 \mathrm{H}) .{ }^{13} \mathrm{C}$ NMR $\left(125 \mathrm{MHz}, \mathrm{CDCl}_{3}\right) \delta: 159.6,136.3,136.3,135.3$, 132.7, 131.7, 131.3, 131.2, 130.2, 129.8, 127.8, 122.1, 114.1, 90.5, 78.7, 73.1, 55.6, 48.3, 47.6, 47.4. $v_{\max }$ (neat, $\mathrm{cm}^{-1}$ ): 3420, 2918, 2849, 1703, 1608, 1548, 1508, 1488, 1462, 1373, 1248, 1176, 1112, 1073. HRMS (ESI) $\mathrm{m} / z$ calcd for $\mathrm{C}_{27} \mathrm{H}_{23} \mathrm{Br}_{2} \mathrm{~N}_{2} \mathrm{O}_{6}$ ([M-H] $]^{-}$): 628.9928; found: 628.9925 . 
Enantiomeric excess of $\mathbf{4 m}$ was determined by chiral stationary phase HPLC analysis using a ChiralPak IC column (90:10 hexanes $/ i-\mathrm{PrOH}$ at $1.0 \mathrm{~mL} / \mathrm{min}, \lambda=220 \mathrm{~nm}$ ), minor enantiomer: $\mathrm{t}_{\mathrm{R}}=$ 16.0 min, major enantiomer: $\mathrm{t}_{\mathrm{R}}=55.5 \mathrm{~min}$.

$\left(1^{\prime} R, 2^{\prime} S, 3\right.$ ' $\left.S, 4^{\prime} S\right)$-3'-Nitro-5'-[(R)-2-nitro-1-phenylethyl]-1',2',3',4'-tetrahydro-[1,1':2',1'"terphenyl]-4'-yl acetate (6)

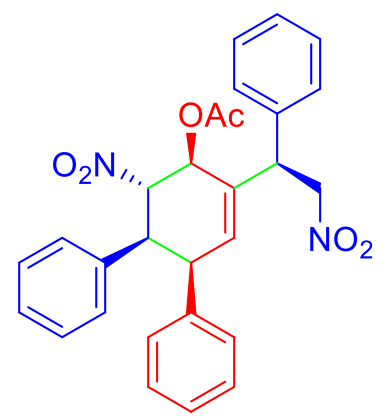

The crude material was purified by flash column chromatography using hexanes/EtOAc (93:7) as an eluent to get $\mathbf{6}$ as a white solid, $38.7 \mathrm{mg}, 80 \%$ yield, m.p. $181-183{ }^{\circ} \mathrm{C} ;{ }^{1} \mathrm{H}$ NMR $\left(500 \mathrm{MHz}, \mathrm{CDCl}_{3}\right) \delta 7.40(\mathrm{t}, J=7.4 \mathrm{~Hz}$, 2H), $7.36-7.31(\mathrm{~m}, 1 \mathrm{H}), 7.29(\mathrm{~d}, J=7.1 \mathrm{~Hz}, 2 \mathrm{H}), 7.23(\mathrm{~d}, J=7.3 \mathrm{~Hz}$, $1 \mathrm{H}), 7.18(\mathrm{t}, J=10.0 \mathrm{~Hz}, 2 \mathrm{H}), 7.13(\mathrm{t}, J=7.4 \mathrm{~Hz}, 1 \mathrm{H}), 7.06(\mathrm{t}, J=7.5 \mathrm{~Hz}$, $2 \mathrm{H}), 6.68(\mathrm{~d}, J=7.1 \mathrm{~Hz}, 2 \mathrm{H}), 6.59(\mathrm{br}, 2 \mathrm{H}), 6.34(\mathrm{~d}, J=8.4 \mathrm{~Hz}, 1 \mathrm{H}), 6.08$ $(\mathrm{d}, J=5.7 \mathrm{~Hz}, 1 \mathrm{H}), 5.23\left(\mathrm{dd}, J_{1}=13.0 \mathrm{~Hz}, J_{2}=8.4 \mathrm{~Hz}, 1 \mathrm{H}\right), 4.87\left(\mathrm{dd}, J_{1}=13.4 \mathrm{~Hz}, J_{2}=8.8 \mathrm{~Hz}\right.$, $1 \mathrm{H}), 4.68\left(\mathrm{dd}, J_{1}=13.4 \mathrm{~Hz}, J_{2}=7.6 \mathrm{~Hz}, 1 \mathrm{H}\right), 4.43(\mathrm{t}, J=8.2 \mathrm{~Hz}, 1 \mathrm{H}), 3.94\left(\mathrm{dd}, J_{1}=13.0 \mathrm{~Hz}, J_{2}\right.$ $=5.8 \mathrm{~Hz}, 1 \mathrm{H}), 3.85(\mathrm{t}, J=5.7 \mathrm{~Hz}, 1 \mathrm{H}), 1.84(\mathrm{~s}, 3 \mathrm{H}) .{ }^{13} \mathrm{C} \mathrm{NMR}\left(125 \mathrm{MHz}, \mathrm{CDCl}_{3}\right) \delta 170.4,137.4$, 136.5, 135.8, 134.3, 131.0, 130.3, 129.6, 128.6, 128.5, 128.4, 128.1, 128.0, 87.3, 78.5, 73.8, 48.4, 47.8, 46.0, 20.7. $v_{\max }\left(\right.$ neat, $\mathrm{cm}^{-1}$ ): 3028, 1739, 1547, 1491, 1453, 1427, 1368, 1271, 1209, 1087, 1015. HRMS (ESI) $\mathrm{m} / \mathrm{z}$ calcd for $\mathrm{C}_{28} \mathrm{H}_{30} \mathrm{~N}_{3} \mathrm{O}_{6}\left(\left[\mathrm{M}+\mathrm{NH}_{4}\right]^{+}\right)$: 504.2129; found: 504.2132. Enantiomeric excess of $\mathbf{6}$ was determined by chiral stationary phase HPLC analysis using a ChiralPak IB column (90:10 hexanes $/ i-\mathrm{PrOH}$ at $1.0 \mathrm{~mL} / \mathrm{min}, \lambda=220 \mathrm{~nm})$, minor enantiomer: $\mathrm{t}_{\mathrm{R}}=$ 22.2 min, major enantiomer: $t_{R}=55.3$ min.

(1'R,2'S,3' $S)$-3'-Nitro-5'-((R)-2-nitro-1-phenylethyl)-2',3'-dihydro-[1,1':2',1'-terphenyl]4'(1'H)-one (7) 


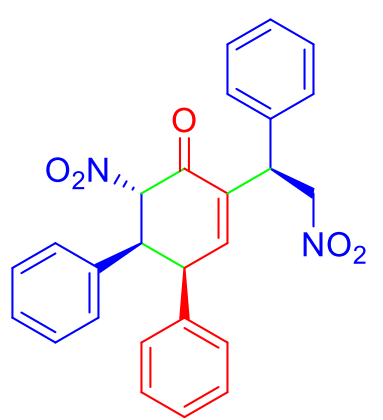

The crude material was purified by flash column chromatography using hexanes/EtOAc (92:8) as an eluent to get 7 as a white solid, $37.6 \mathrm{mg}, 85$ $\%$ yield, m.p. $125-127{ }^{\circ} \mathrm{C},{ }^{1} \mathrm{H}$ NMR $\left(500 \mathrm{MHz}, \mathrm{CDCl}_{3}\right) \delta$ 7.40-7.33 (m, $5 \mathrm{H}), 7.25-7.05(\mathrm{~m}, 7 \mathrm{H}), 6.65(\mathrm{~d}, J=7.5 \mathrm{~Hz}, 2 \mathrm{H}), 6.56(\mathrm{~d}, J=7.6 \mathrm{~Hz}$, $2 \mathrm{H}), 5.78(\mathrm{~d}, J=14.1 \mathrm{~Hz}, 1 \mathrm{H}), 5.17\left(\mathrm{dd}, J_{1}=12.7 \mathrm{~Hz}, J_{2}=8.9 \mathrm{~Hz}, 1 \mathrm{H}\right)$, $4.92(\mathrm{t}, J=8.0 \mathrm{~Hz}, 1 \mathrm{H}), 4.84\left(\mathrm{dd}, J_{1}=12.7 \mathrm{~Hz}, J_{2}=7.1 \mathrm{~Hz}, 1 \mathrm{H}\right), 4.41(\mathrm{dd}$, $\left.J_{1}=14.1 \mathrm{~Hz}, J_{2}=5.6 \mathrm{~Hz}, 1 \mathrm{H}\right), 4.11(\mathrm{t}, J=5.8 \mathrm{~Hz}, 1 \mathrm{H}) .{ }^{13} \mathrm{C} \mathrm{NMR}\left(125 \mathrm{MHz}, \mathrm{CDCl}_{3}\right) \delta 187.4$, 148.9, 137.0, 136.9, 135.6, 133.2, 130.0, 129.7, 129.0, 128.9, 128.8, 128.7, 128.5, 128.3, 127.9, 90.4, 76.9, 48.8, 48.5, 44.5. $v_{\max }\left(\right.$ neat, $\mathrm{cm}^{-1}$ ): 1689, 1551, 1493, 1453, 1374, 1205, 1085, 1032, 918, 789, 766, 747. HRMS (ESI) $\mathrm{m} / \mathrm{z}$ calcd for $\mathrm{C}_{26} \mathrm{H}_{26} \mathrm{~N}_{3} \mathrm{O}_{5}\left(\left[\mathrm{M}+\mathrm{NH}_{4}\right]^{+}\right)$: 460.1867; found: 460.1871. Enantiomeric excess of 7 was determined by chiral stationary phase HPLC analysis using a ChiralPak IC column (70:30 hexanes $/ i-\mathrm{PrOH}$ at $1.0 \mathrm{~mL} / \mathrm{min}, \lambda=220 \mathrm{~nm}$ ), minor enantiomer: $t_{R}=25.6 \mathrm{~min}$, major enantiomer: $t_{R}=37.3 \mathrm{~min}$. 


\section{Additional References}

12. a) Schönberger, M.; Trauner, D. Angew. Chem., Int. Ed. 2014, 53, 3264-3267. b) Reddy, B. V. S.; Sundar, C. S.; Reddy, M. R.; Reddy, C. S.; Sridhar B. Synthesis 2015; 47, 1117 1122 .

13. a) Black, P.-J.; Gerta, C.-K.; Edwards, M. G.; Slatford, P. A.; Whittlesey, M. K.; Williams, J. M. J. Org. Biomol. Chem. 2006, 4, 116-125. b) Vo, Q. V.; Trenerry, C.; Rochfort, S.; Wadeson, J.; Leyton, C.;Hughes, A. B. Bioorg. Med. Chem. 2013, 21, 5945-5954.

14. a) Vakulya, B.; Varga, S.; Csámpai, A.; Soós, T. Org. Lett. 2005, 7, 1967-1969; b) Liu, Y.; Sun, B.; Wang, B.; Wakem, M.; Deng, L.; J. Am. Chem. Soc. 2009, 131, 418-419; c) Han, X.; Zhong, F.; Lua, Y. Adv. Synth. Catal. 2010, 352, 2778-2782, d) Yang, W.; Du, D.-M. Org. Lett. 2010, 12, 5450-5453; e) Li, X.; Deng, H.; Zhang, B.; Li, J.; Zhang, L.; S. Luo J.-P. Cheng, Chem. Eur. J. 2010, 16, 450; f) Hajra, S.; Jana, B. Org. Lett. 2017, 19, 47784781, g) Huang, H.; Konda, S.; Zhao, J. C. G. Angew. Chem., Int. Ed. 2016, 55, 2213-2216. h) Pozo, S. Del.; Vera, S.; Oiarbide, M.; Palomo, C. J. Am. Chem. Soc., 2017, 139, 1530815311. i) Tan, W.; Wei, J.; Jiang, X. Org. Lett. 2017, 19, 2166-2169.

15. Wenzel, A. G.; Jacobsen, E. N. J. Am. Chem. Soc. 2002, 124, 12964-12965. 


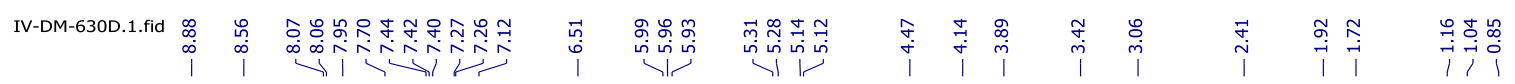

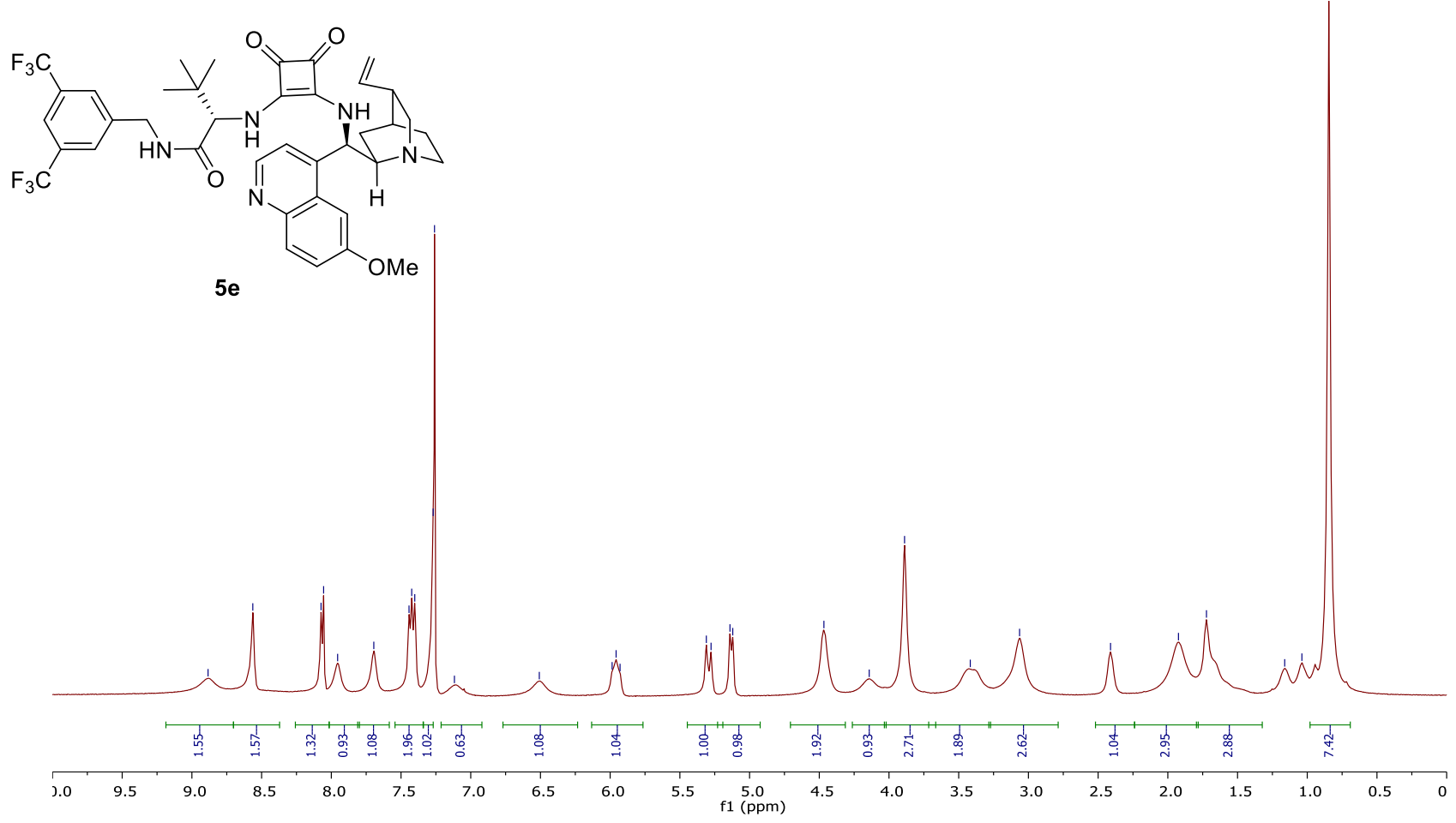

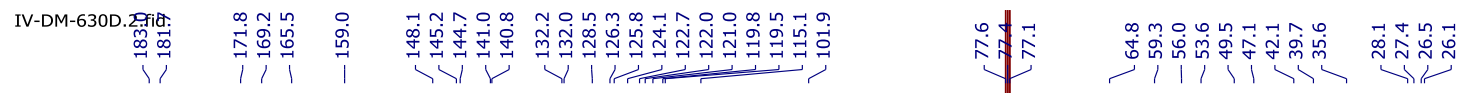

- $11111<1<$ पit

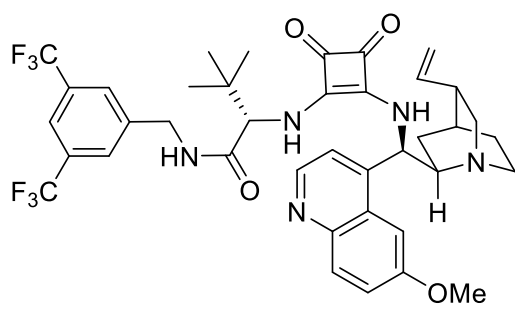

5

$\mathrm{OMe}$

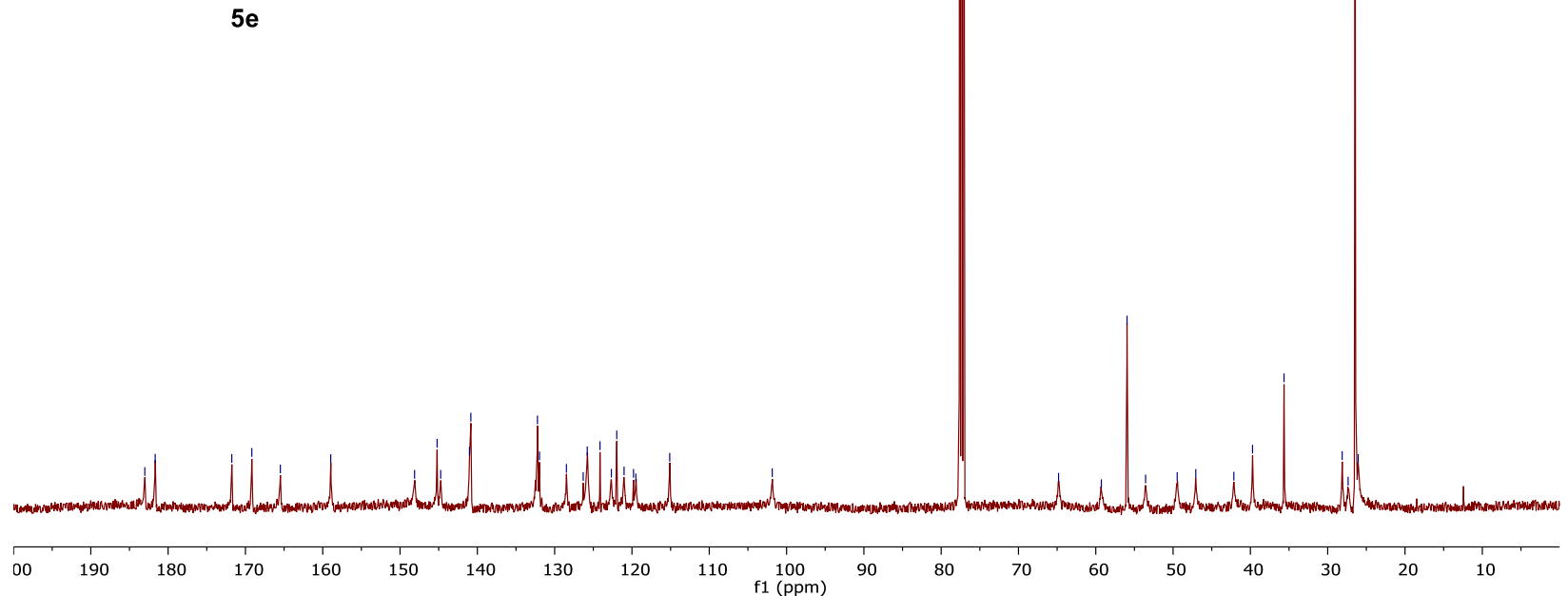




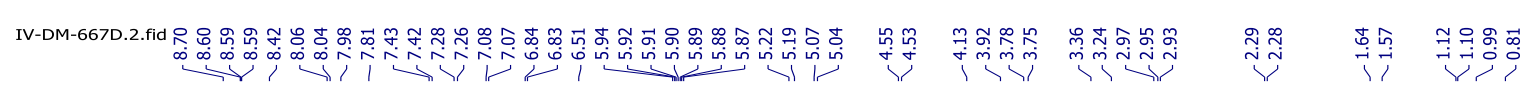

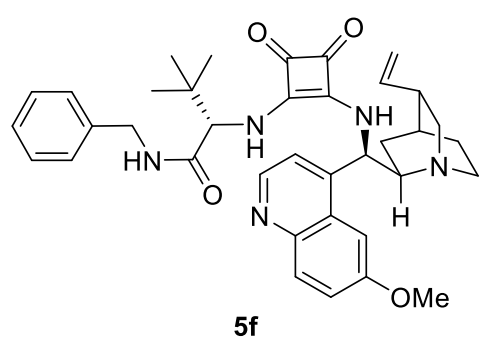

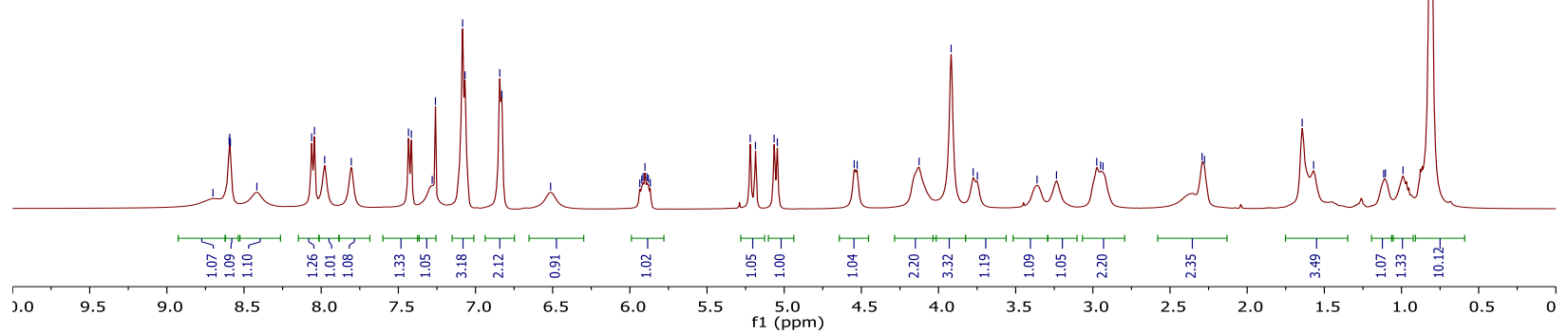

IV-DM-667D.P्य

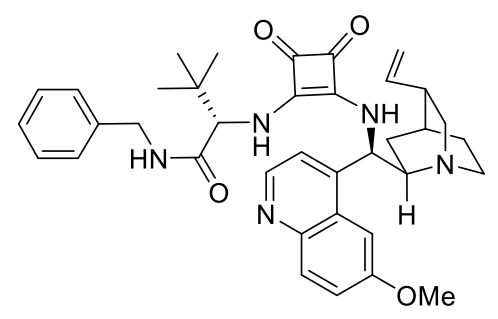

$5 f$

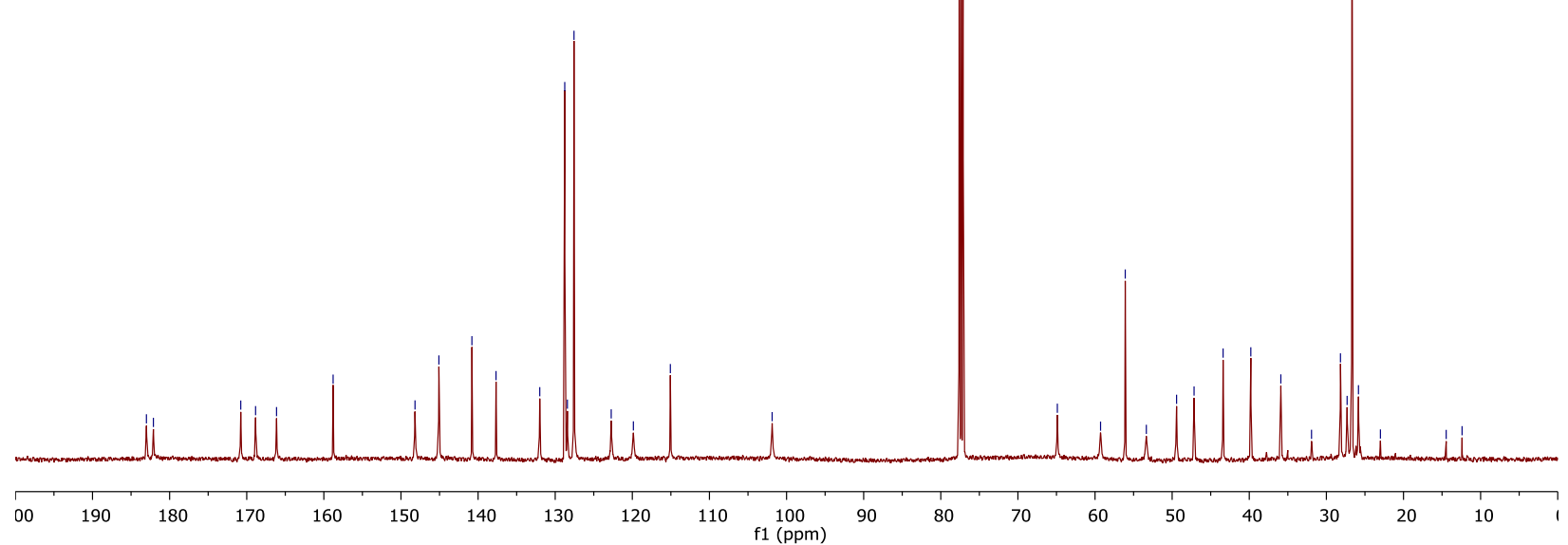


IV-DM-616D.1.fid

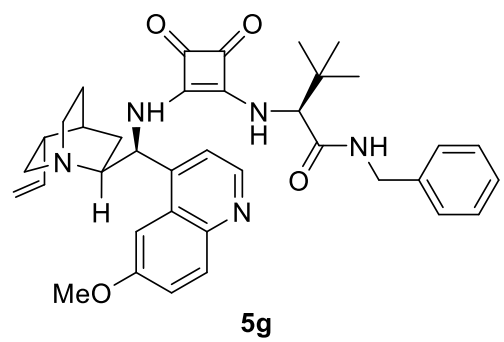

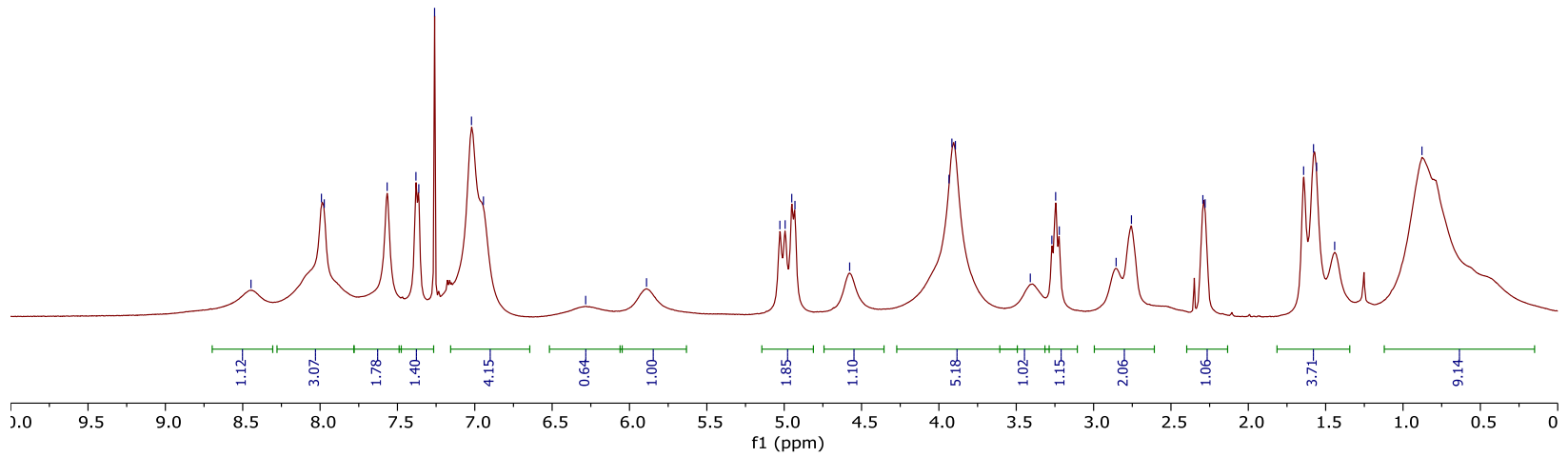
IV-DM-616D.2n⿴囗十)<smiles></smiles>

$5 g$ 

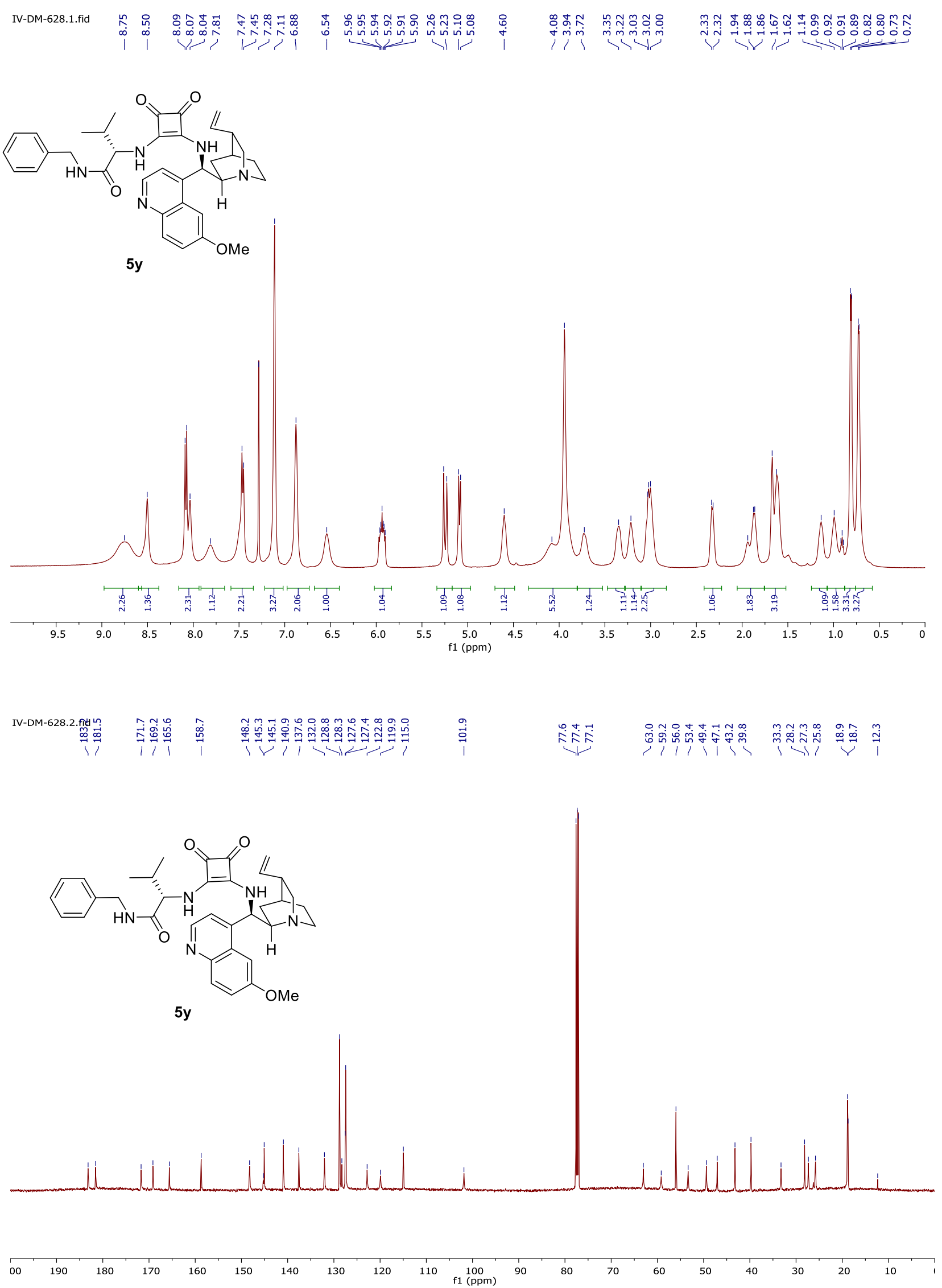


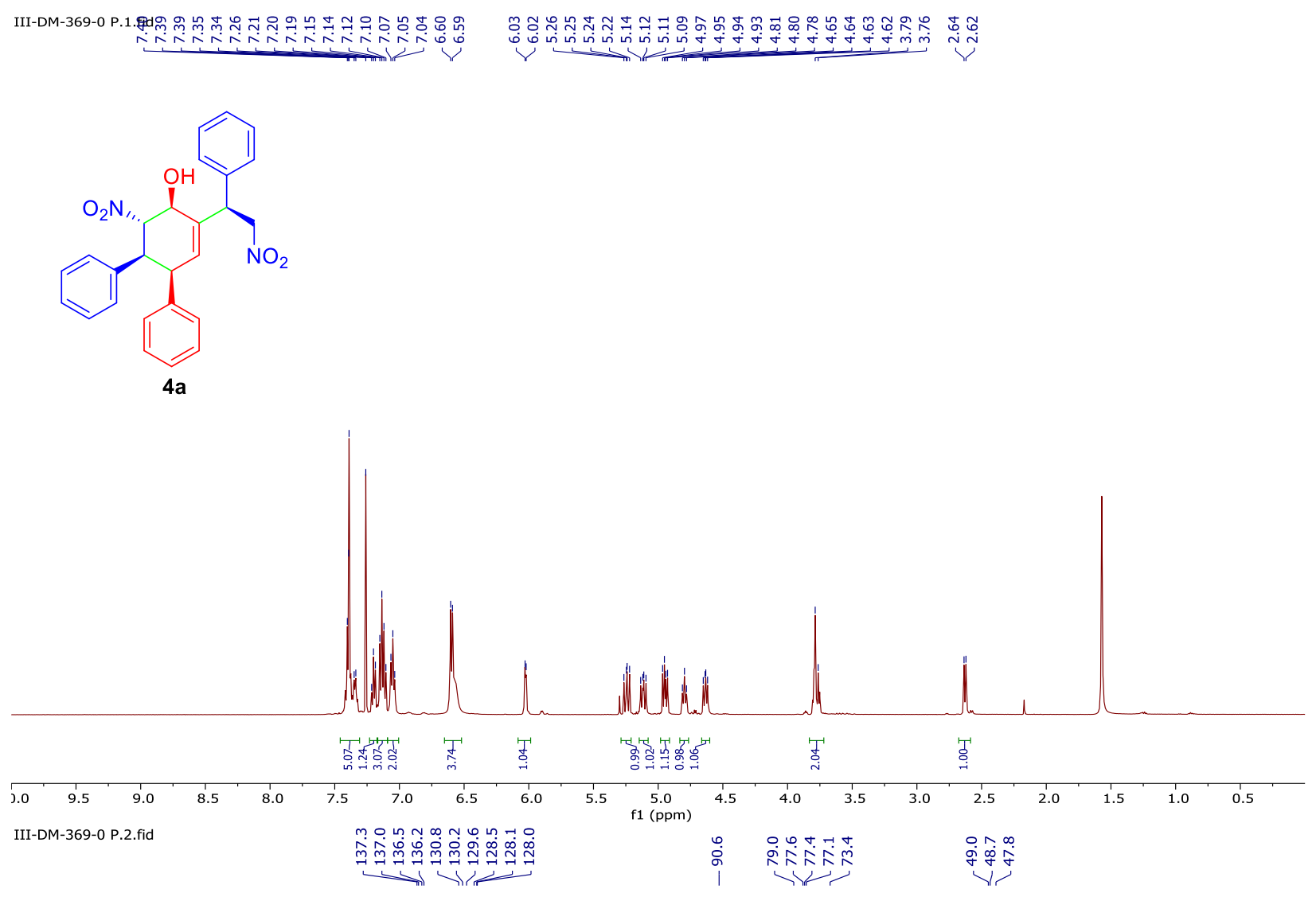<smiles>O=[N+]([O-])CC(C1=CC(c2ccccc2)[C@H](c2ccccc2)[C@H](O)[C@H]1O)c1ccccc1</smiles>

$4 a$

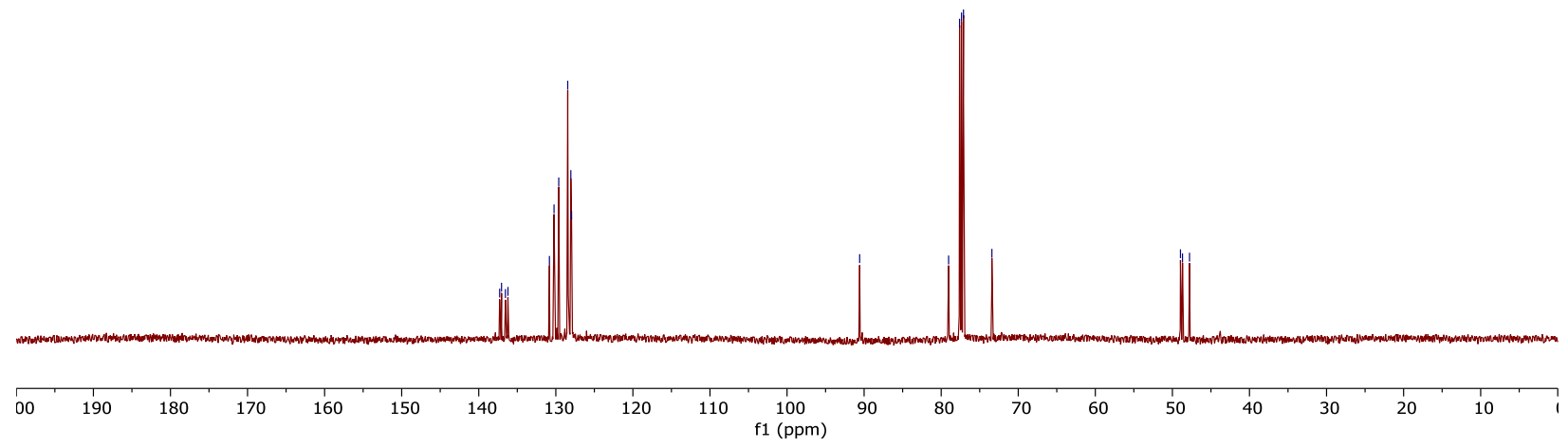


<smiles></smiles>

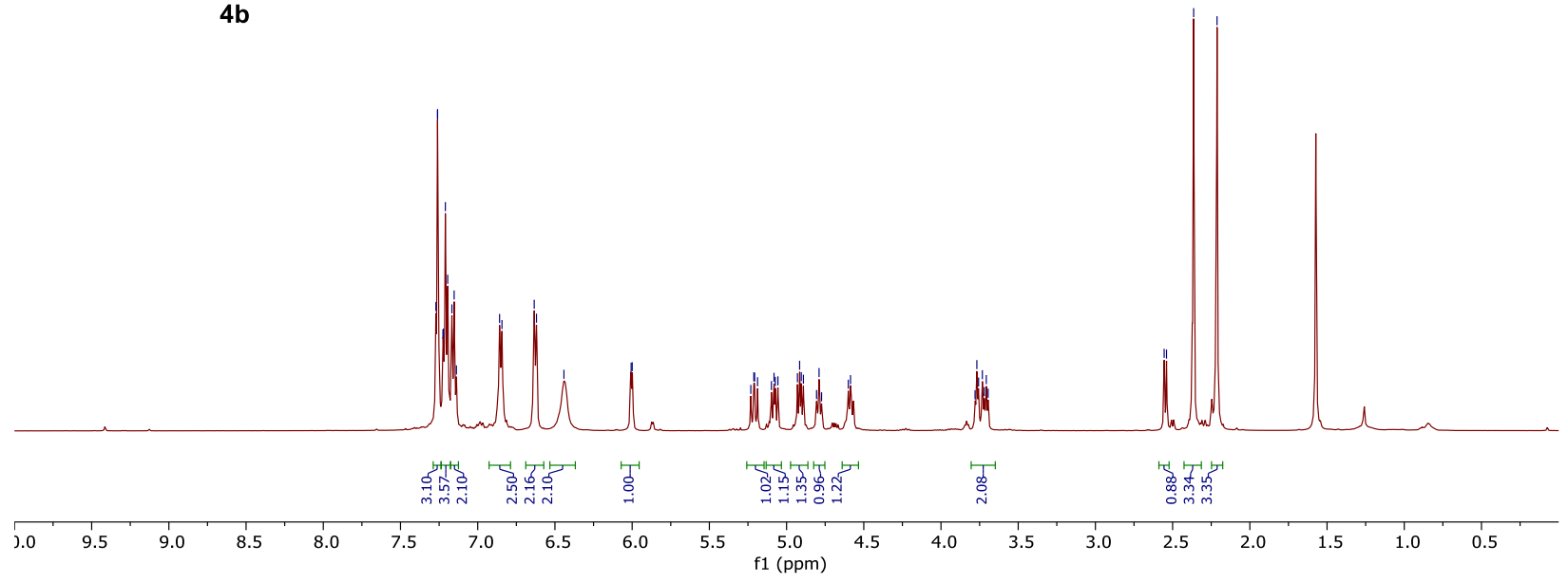

IV-DM-669A.5.fid<smiles>Cc1ccc(C(C[N+](=O)[O-])C2C=C(C3=CC=[12C+]C=C3)C(c3ccc(C)cc3)[C@H]([N+](=O)[O-])[C@H]2O)cc1</smiles>

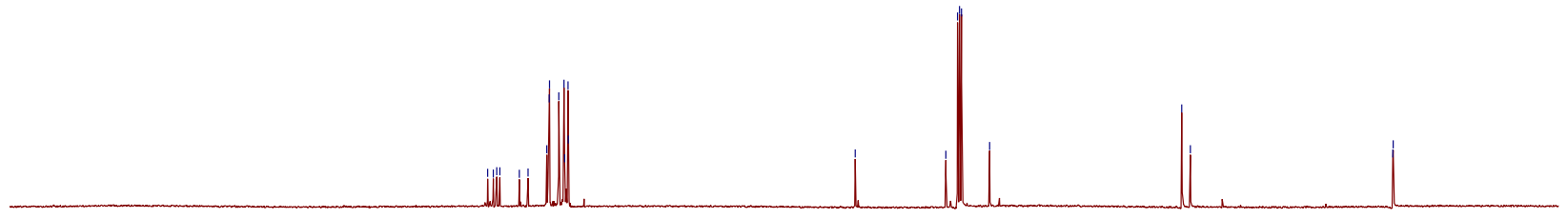




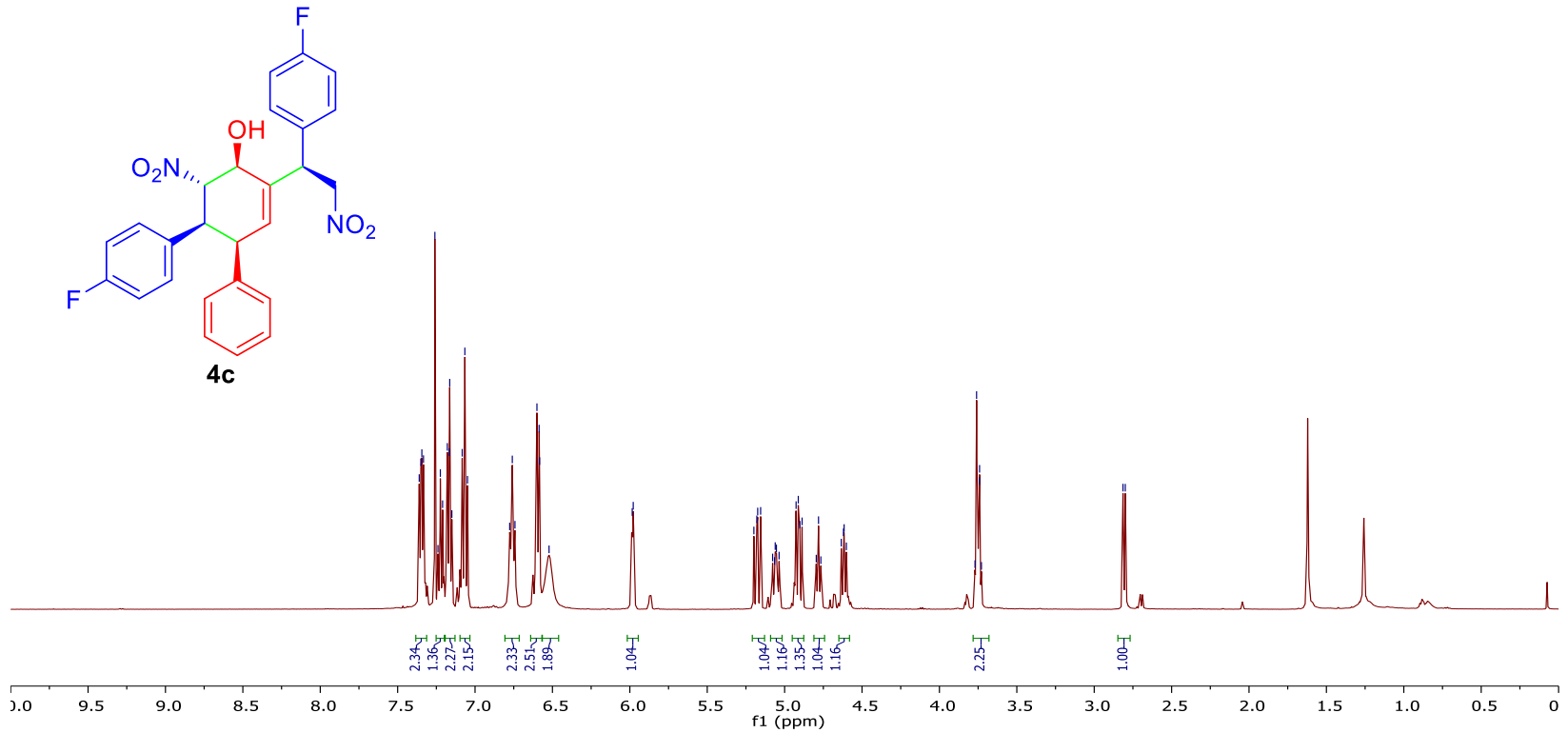<smiles></smiles>

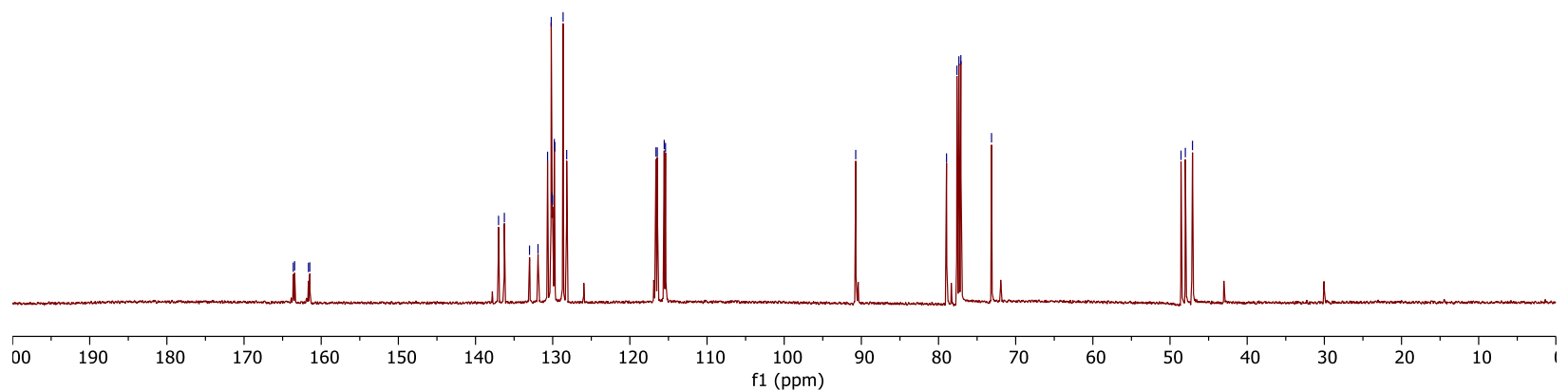



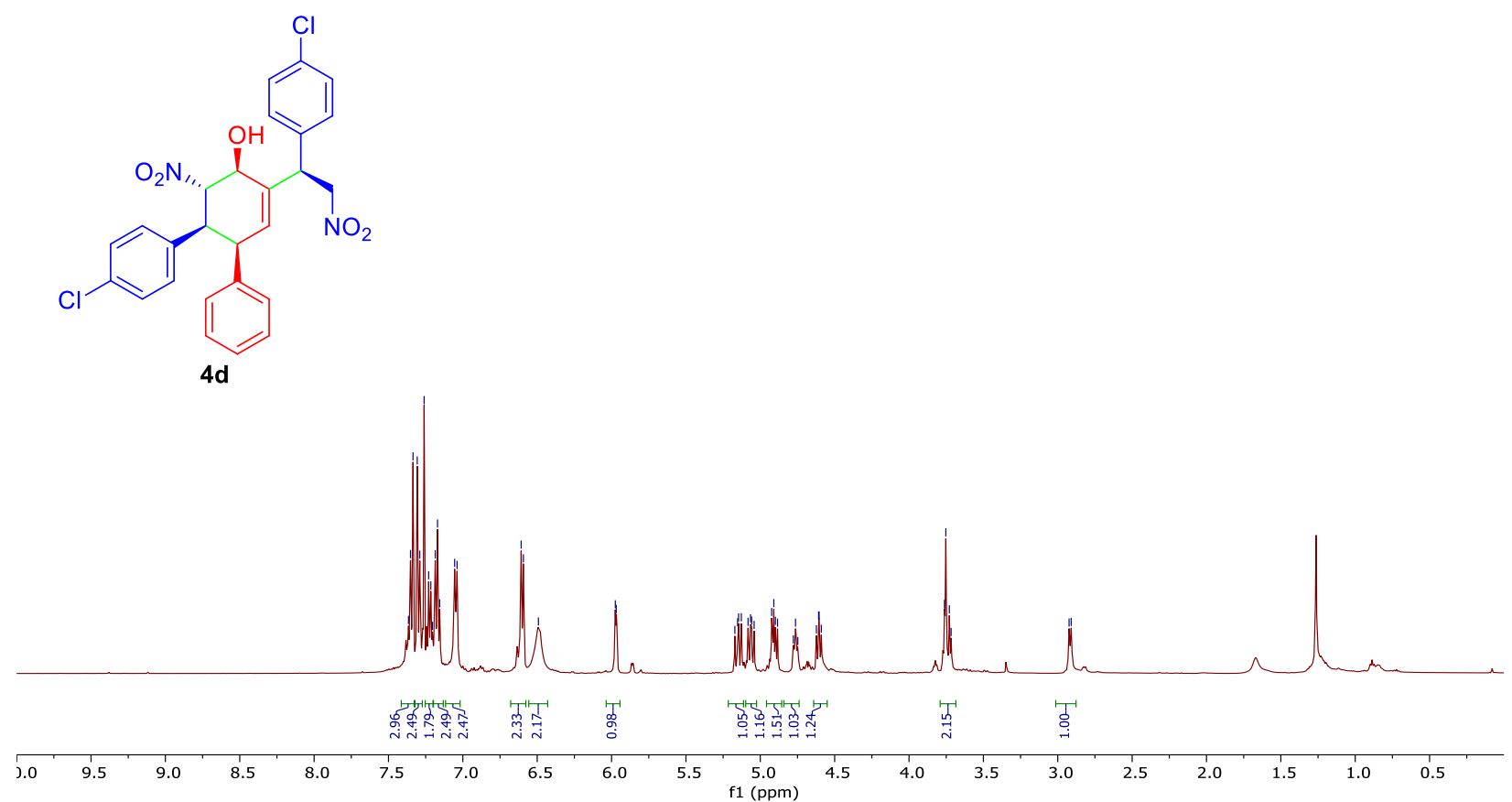

1-SJ-569-P2-C13.1.fid<smiles></smiles>

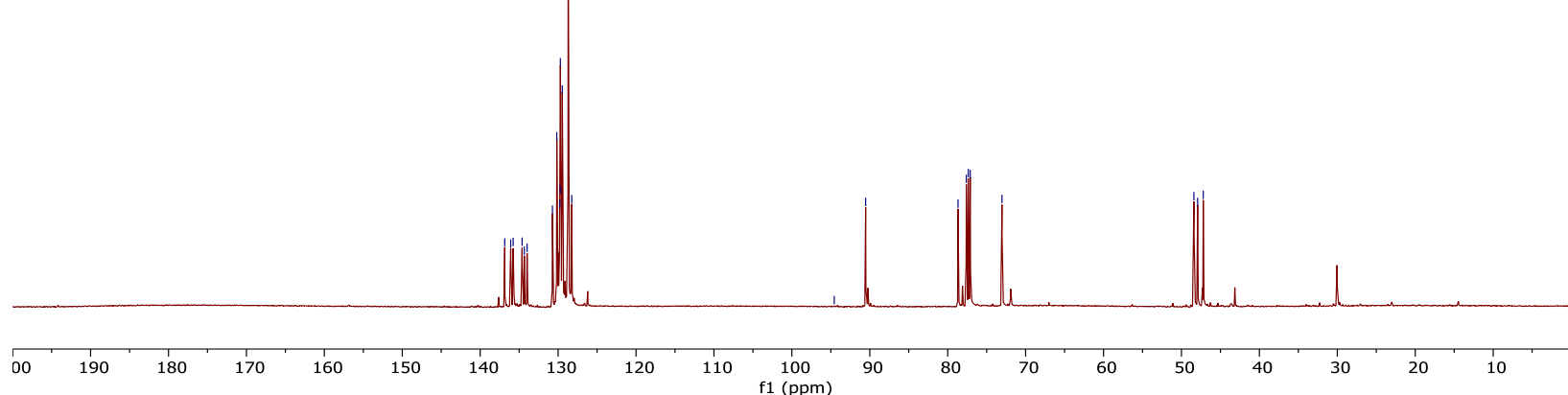



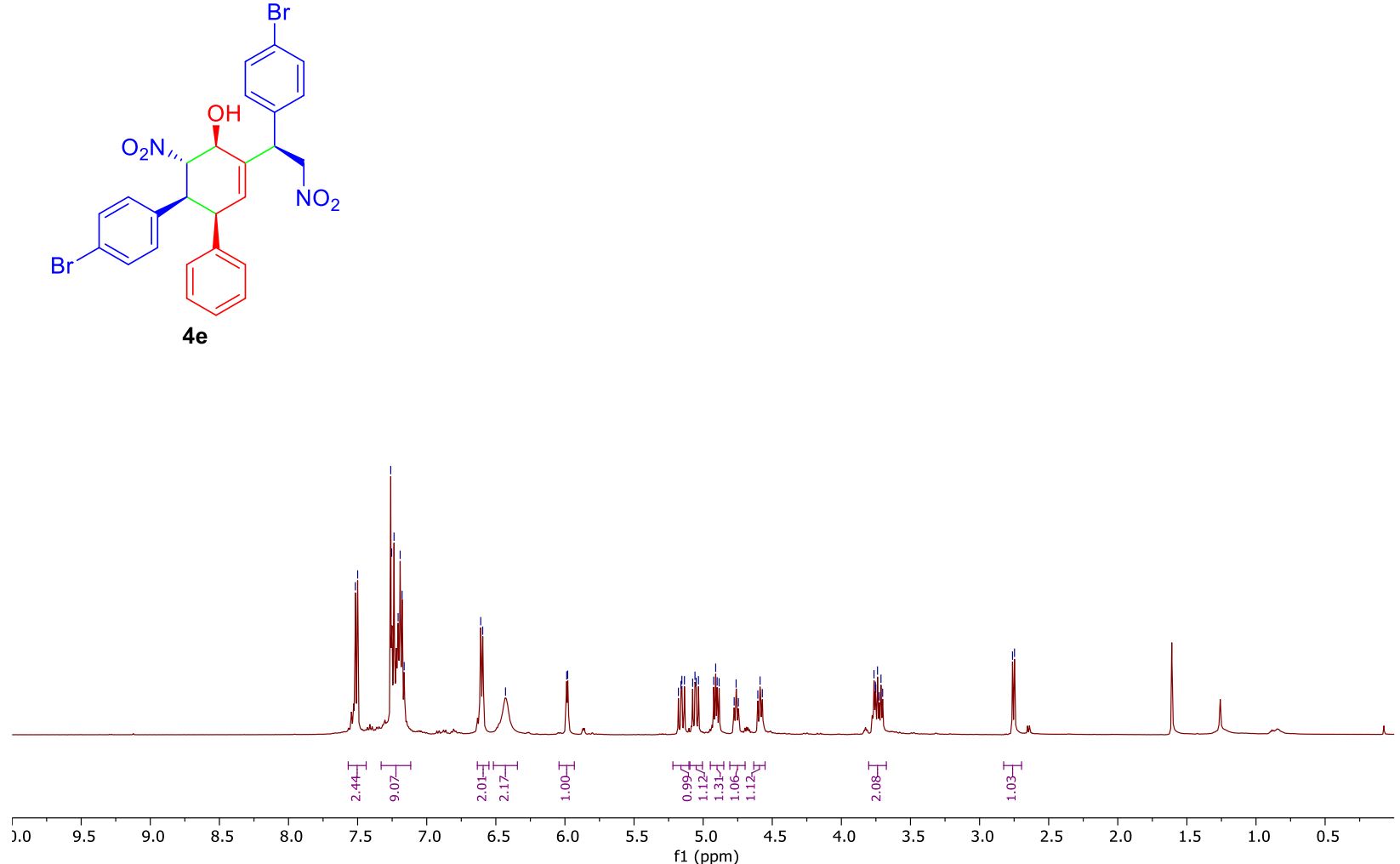

V-DM-714D2.2.fid

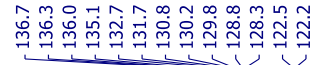

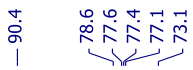

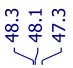<smiles>O=[N+]([O-])CC(C1=CC(c2ccccc2)C(c2ccc(Br)cc2)C(O)C1[N+](=O)[O-])c1ccc(Br)cc1</smiles>

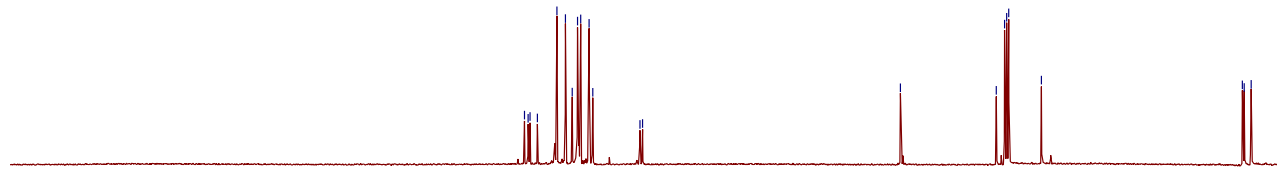

$190 \quad 180 \quad 170 \quad 160$

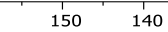

$130-120$

$110 \begin{gathered}100 \\ f 1(\mathrm{ppm})\end{gathered}$

$90 \quad 80,70,60$

$\begin{array}{lllll}50 & 10 & 30 & 20 & 10\end{array}$ 


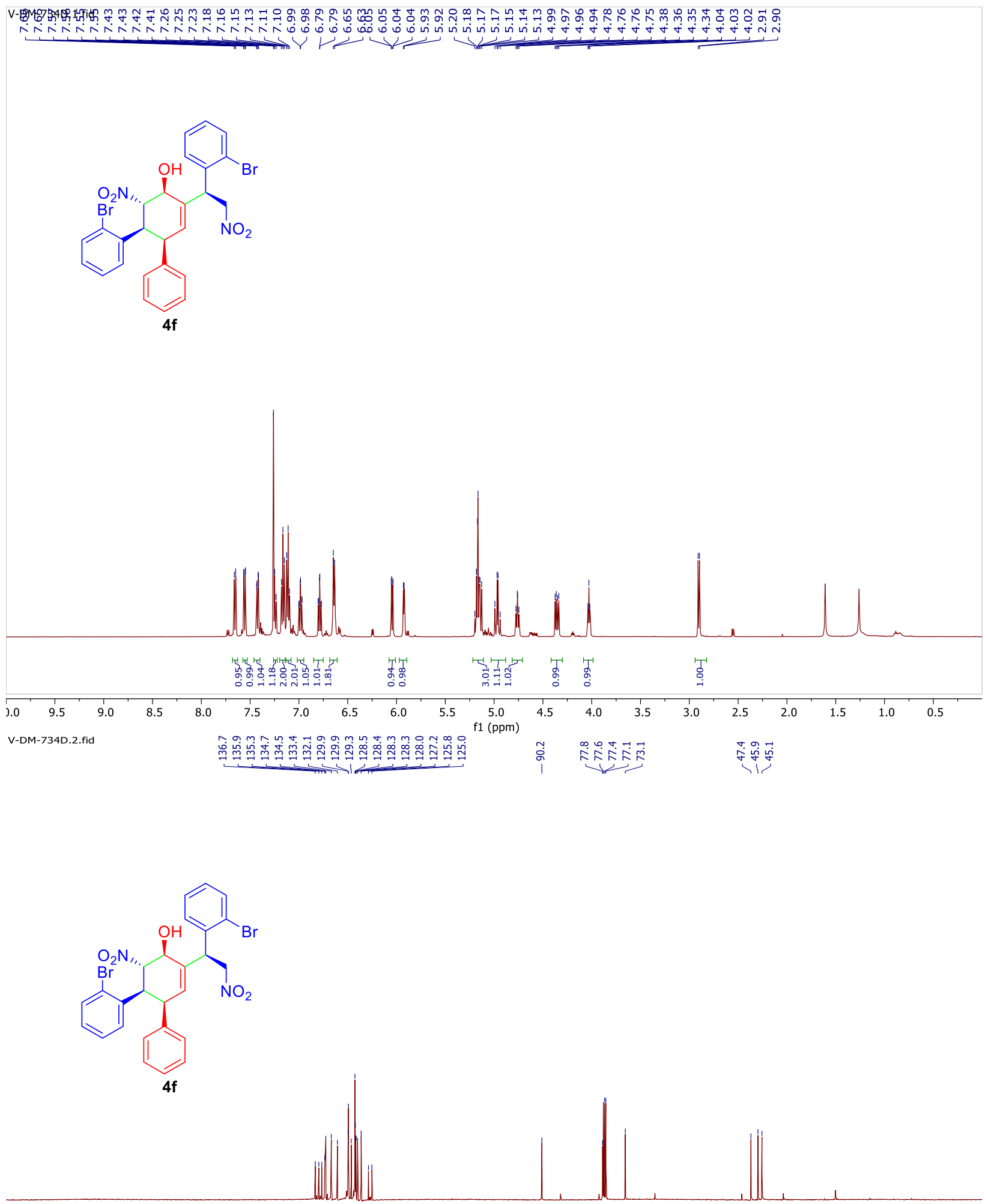

oo

$190 \quad 180$

160

$130 \quad 120$

100
f1 $(\mathrm{ppm})$ 


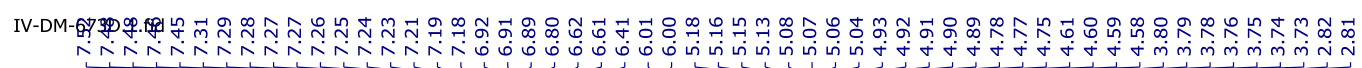<smiles>O=[N+]([O-])C[C@H](C1=C[C@H](c2ccc([Ge])cc2)[C@H](c2cccc(Br)c2)[C@@H]([N+](=O)[O-])[C@H]1O)c1cccc(Br)c1</smiles>

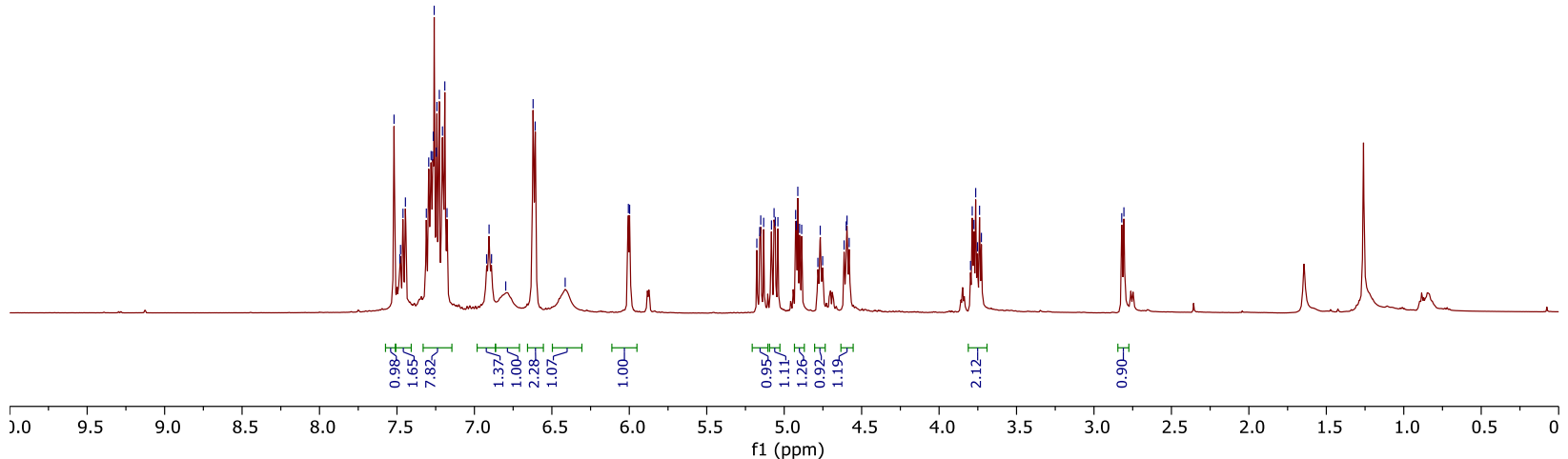

IV-DM-673D.2.fid<smiles>[14CH3]c1ccc(C2C=C(C(C[N+](=O)[O-])c3cccc(Br)c3)[C@H](O)[C@@H]([N+](=O)[O-])[C@H]2c2cccc(Br)c2)cc1</smiles>

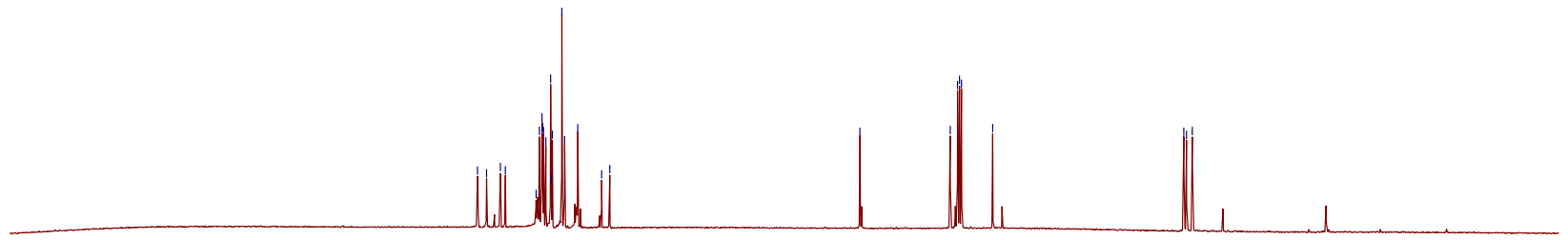

1 $190+170$

$150 \quad 140 \quad 130 \quad 120$

100
f1 
<smiles>O=[N+]([O-])CC(C1=CC(c2ccccc2)C(c2cccs2)C(O)[C@H]1[N+](=O)[O-])c1cccs1</smiles>

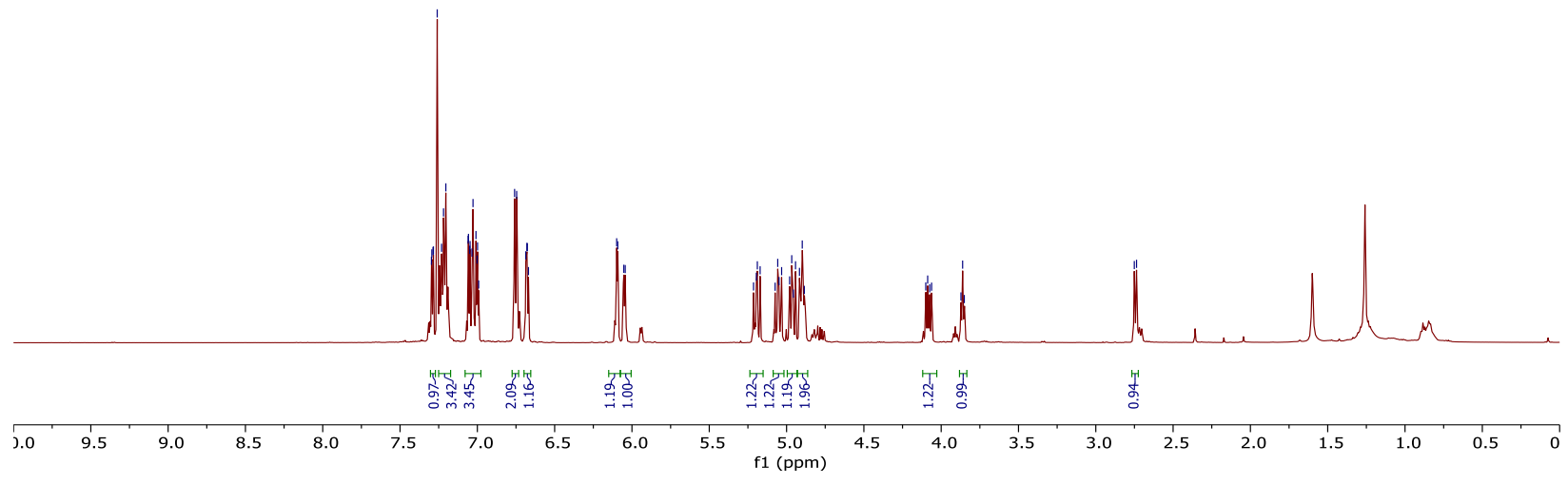

IV-DM-677A.5.fid

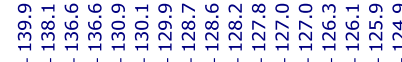

l

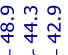<smiles>O=[N+]([O-])CC(C1=CC(c2ccccc2)[C@@H](c2cccs2)[C@H](O)[C@H]1O)c1cccs1</smiles>

4h

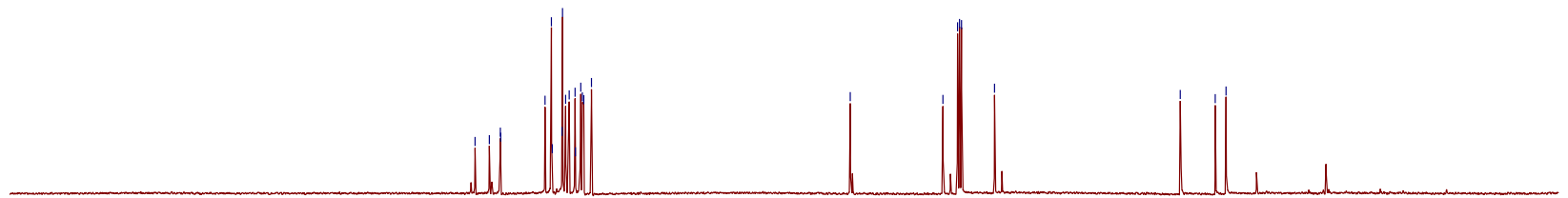

0

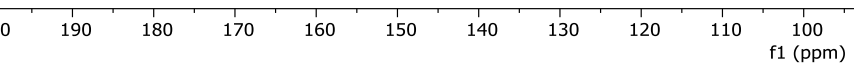
$\begin{array}{lllllllll}1 & 1 & 1 & 1 & 1 & 1 & 1 & 1 & 1\end{array}$ 


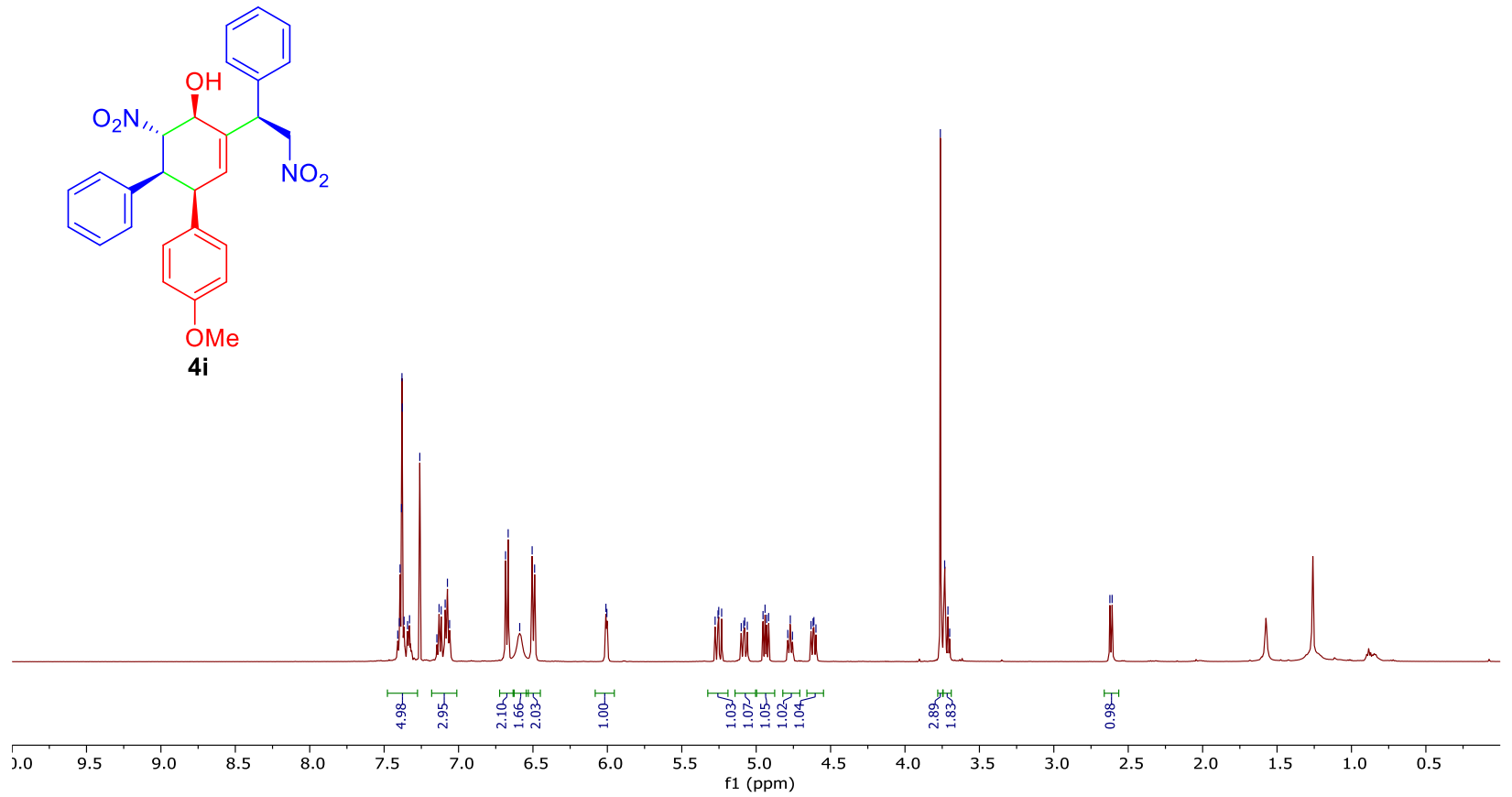

V-DM-724.4.fid
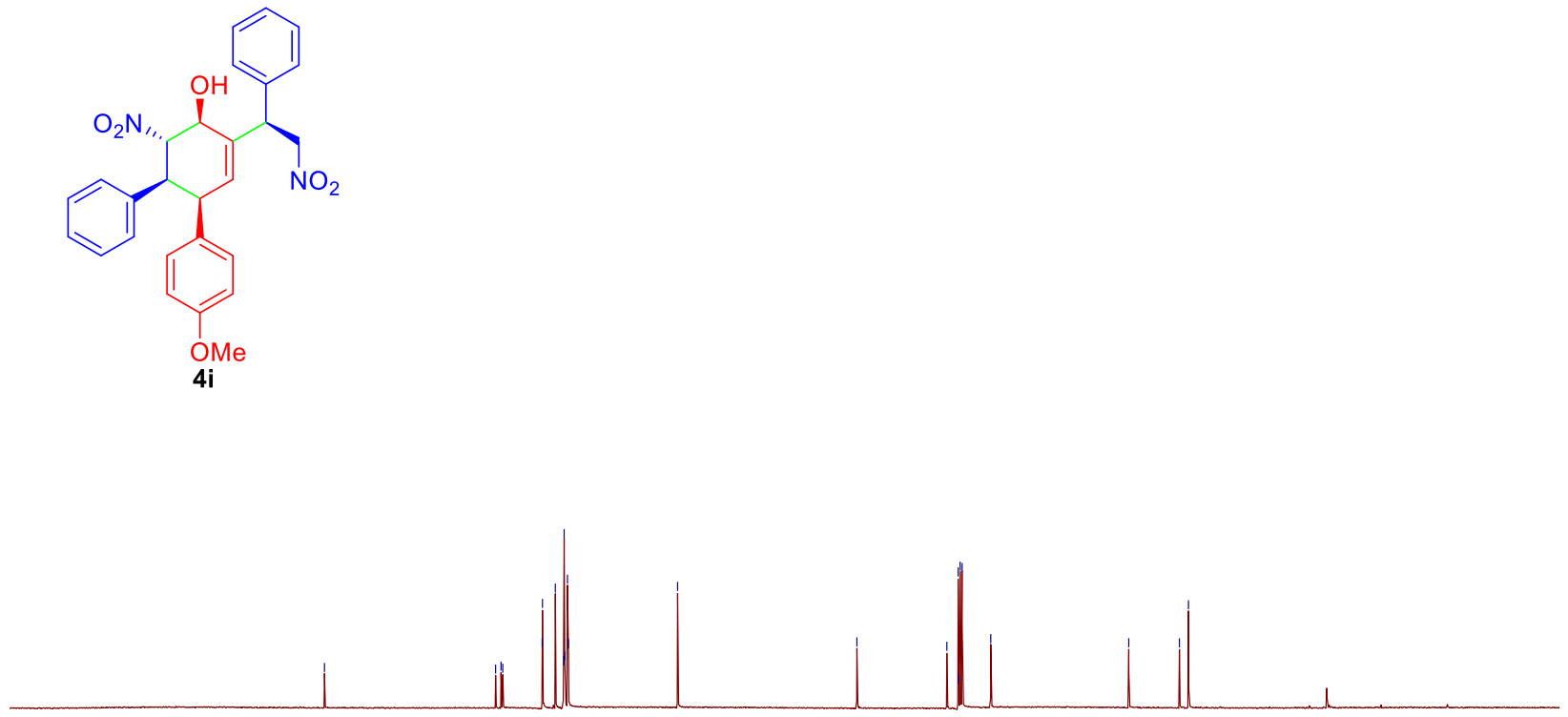

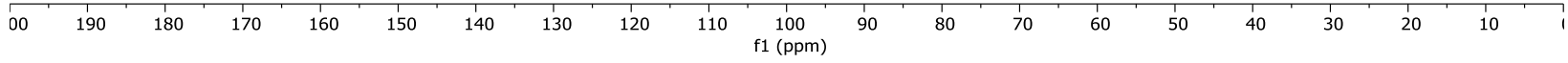



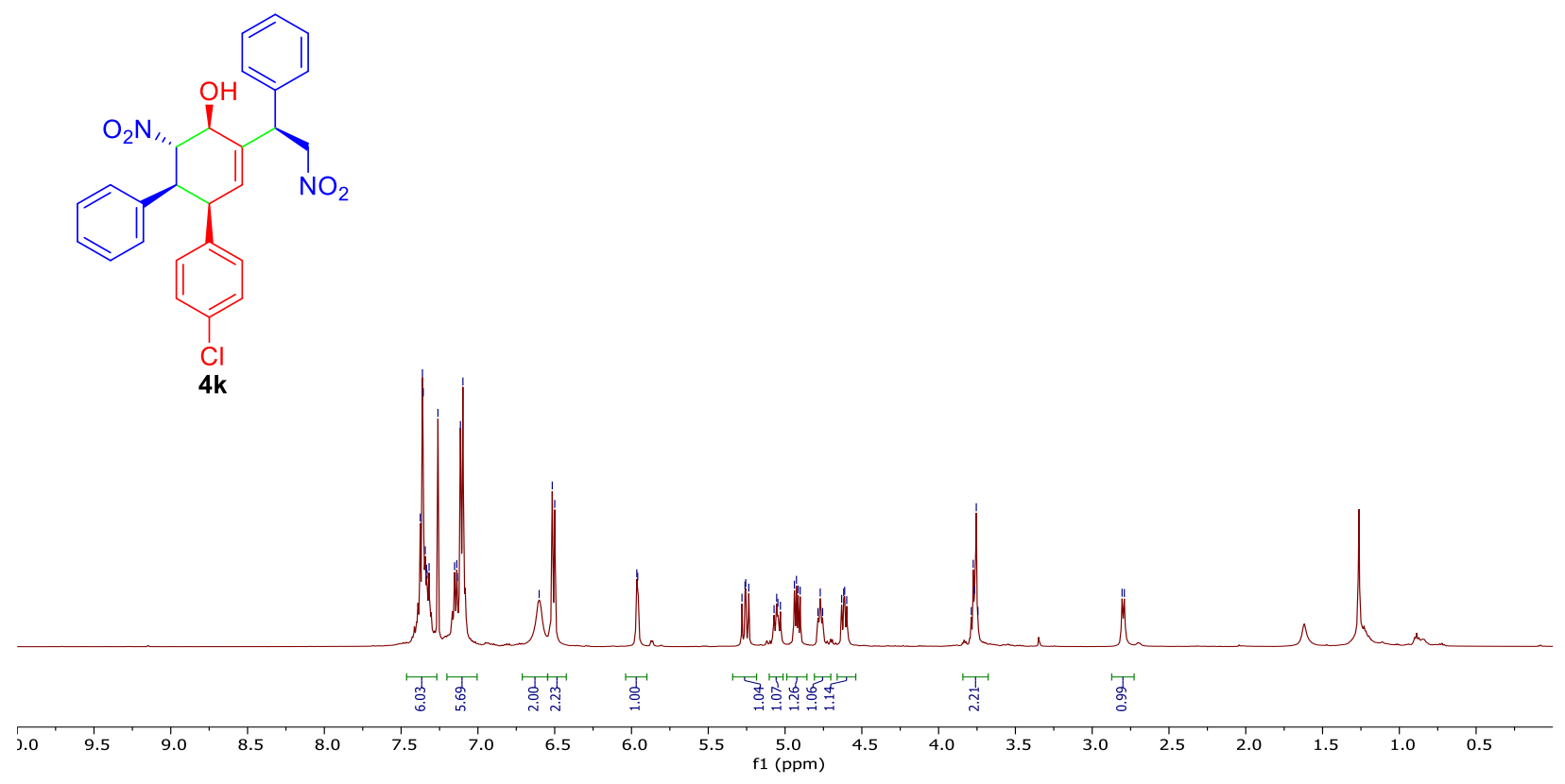

1-SJ-614-PP-C13.1.fid

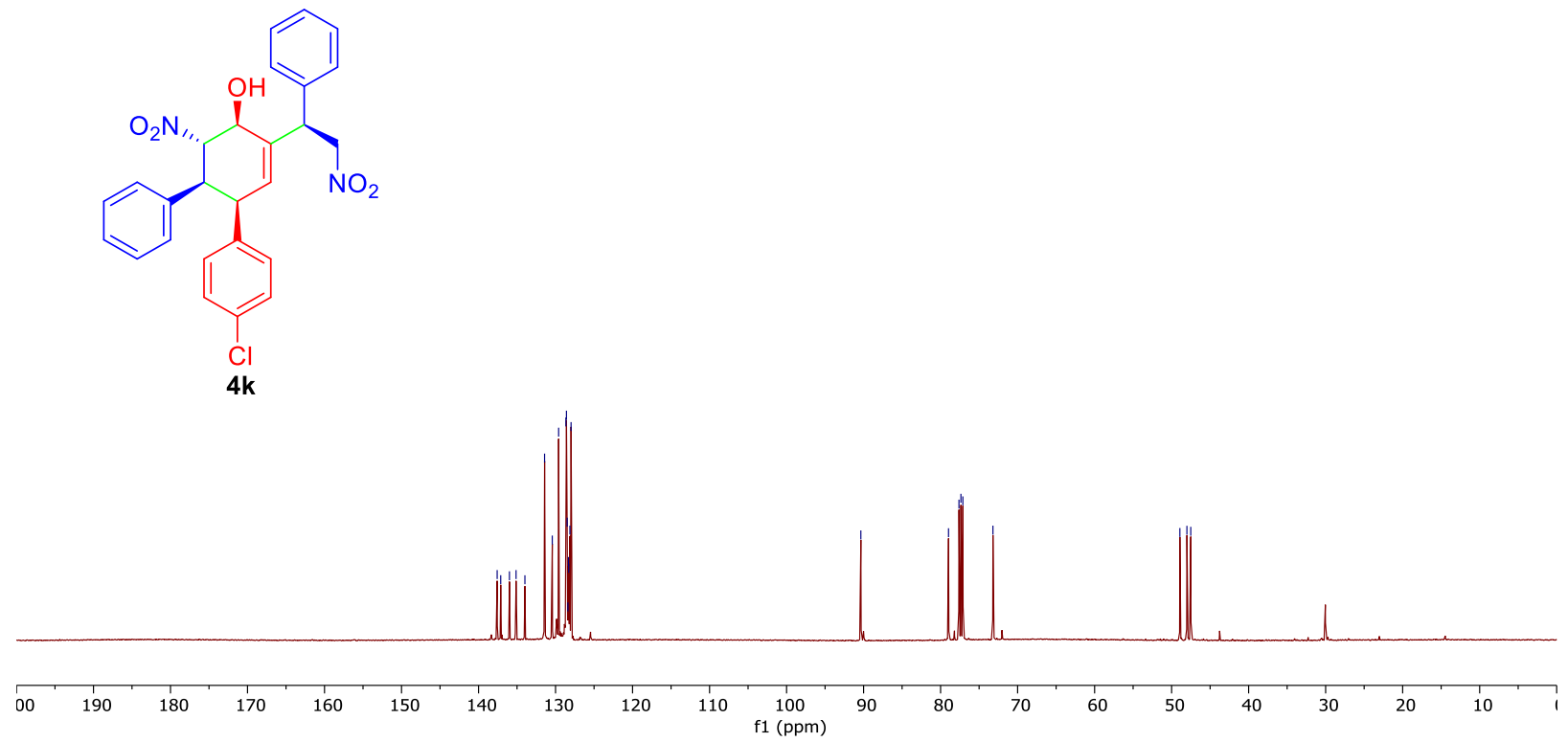




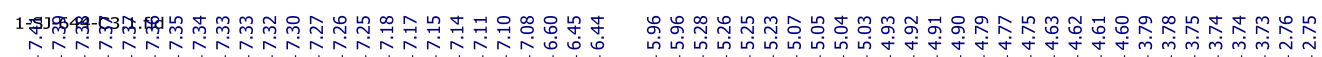

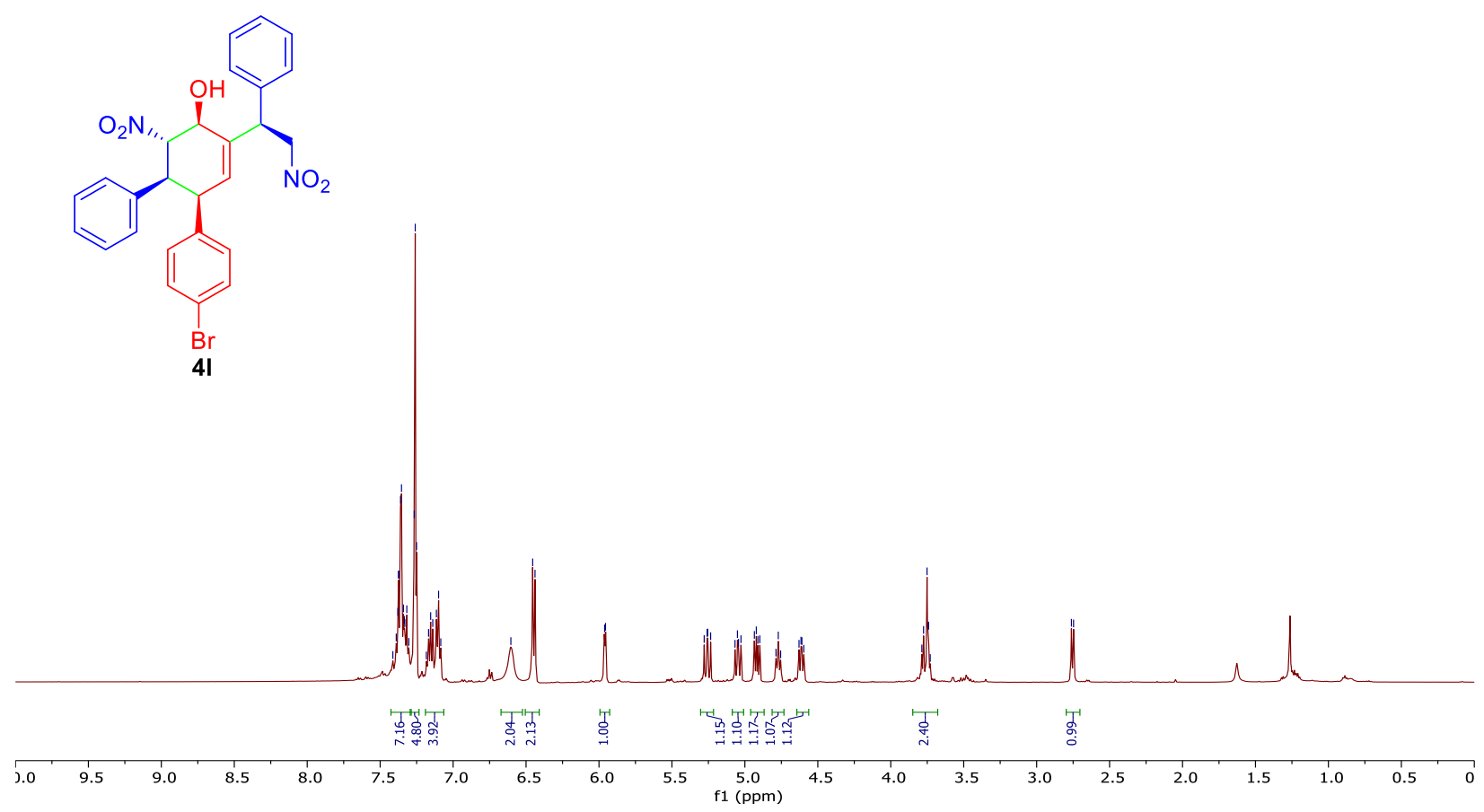

1-SJ-644-C3-C13.1.fid<smiles>O=[N+]([O-])CC(C1=CC(c2ccc(Br)cc2)C(c2ccccc2)[C@H]([N+](=O)[O-])C1O)c1ccccc1</smiles>

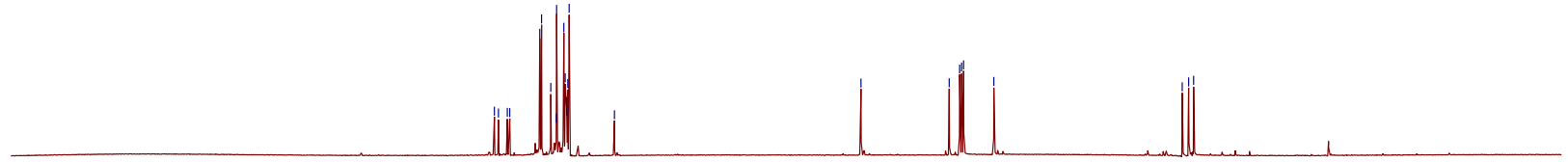

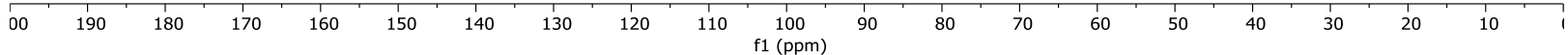



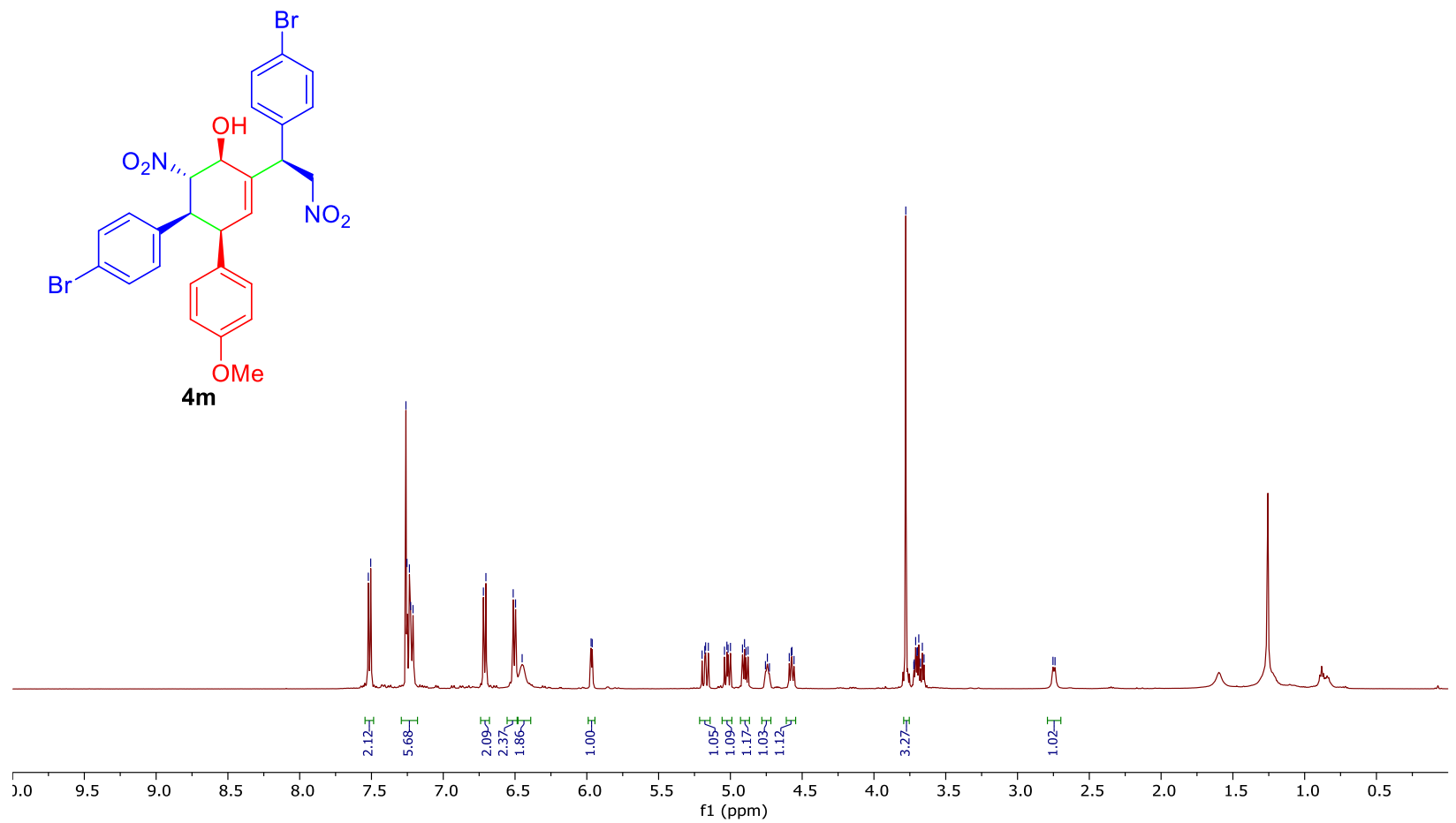
V-DM-751D.2.fid 兽<smiles>COc1ccc(C2C=C(C(C[N+](=O)[O-])c3ccc(Br)cc3)[C@H](O)[C@H]([N+](=O)[O-])[C@H]2c2ccc(Br)cc2)cc1</smiles>

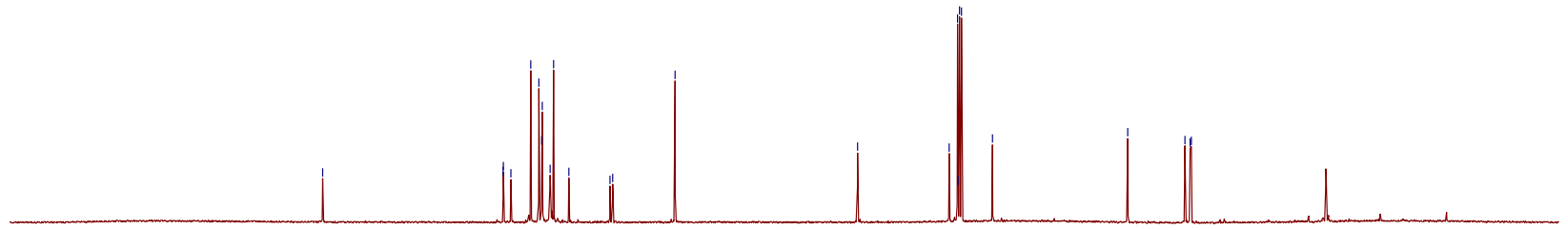


<smiles></smiles>

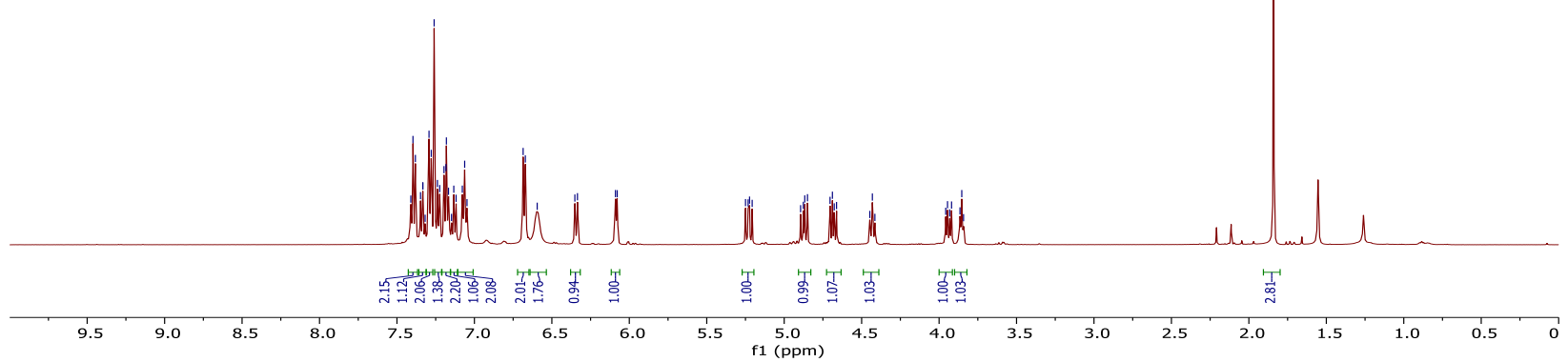<smiles></smiles>

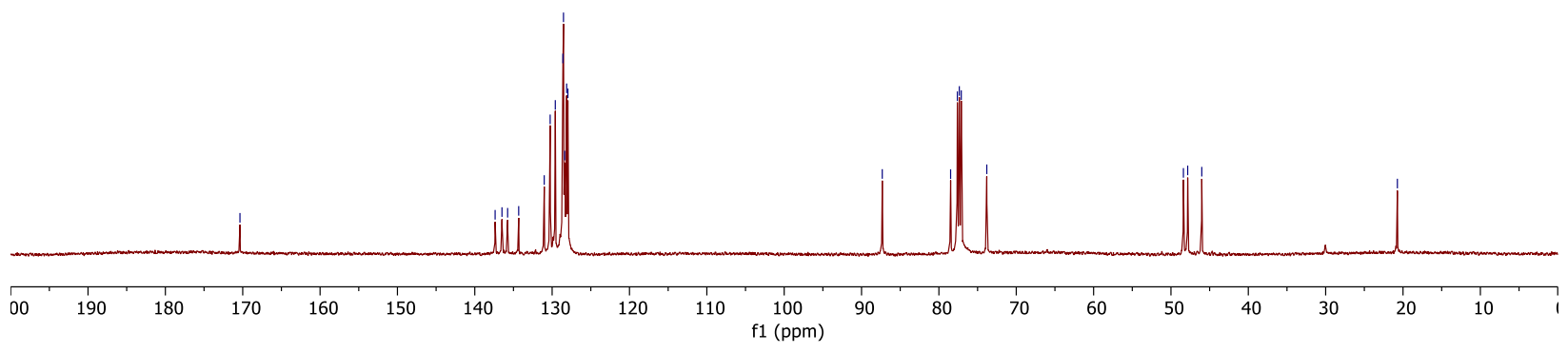



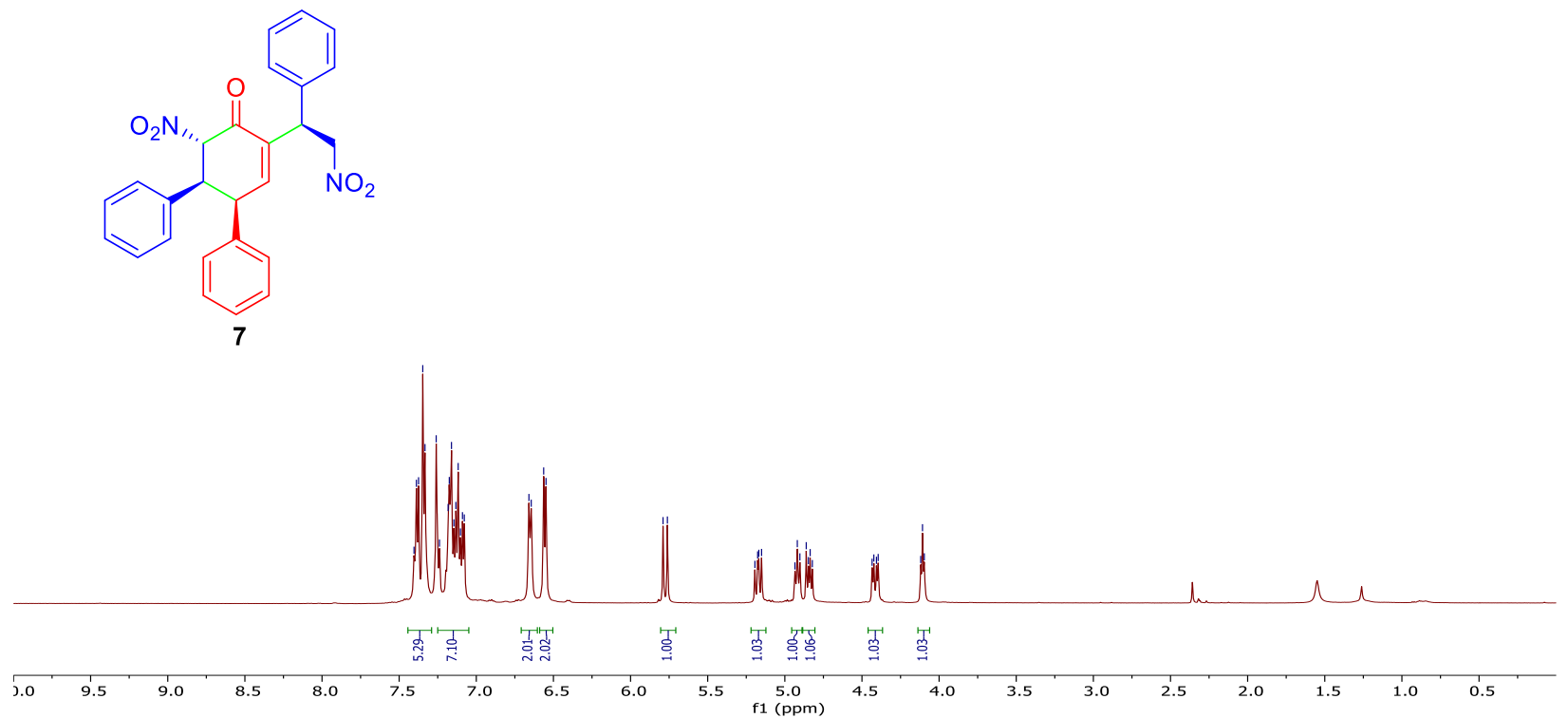
V-DM-875 Did. fid $\left.\begin{array}{l}a \\ 0\end{array}\right)$<smiles></smiles>

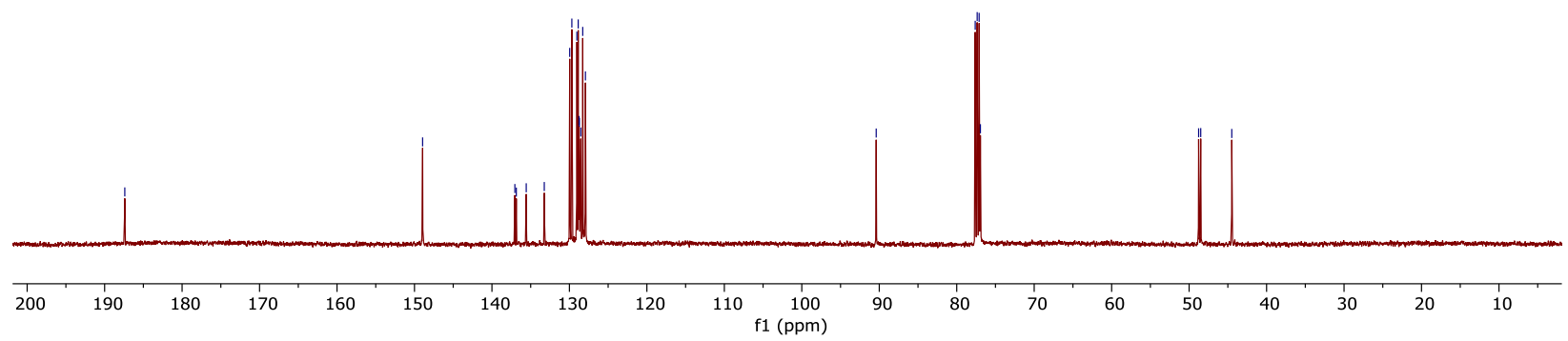



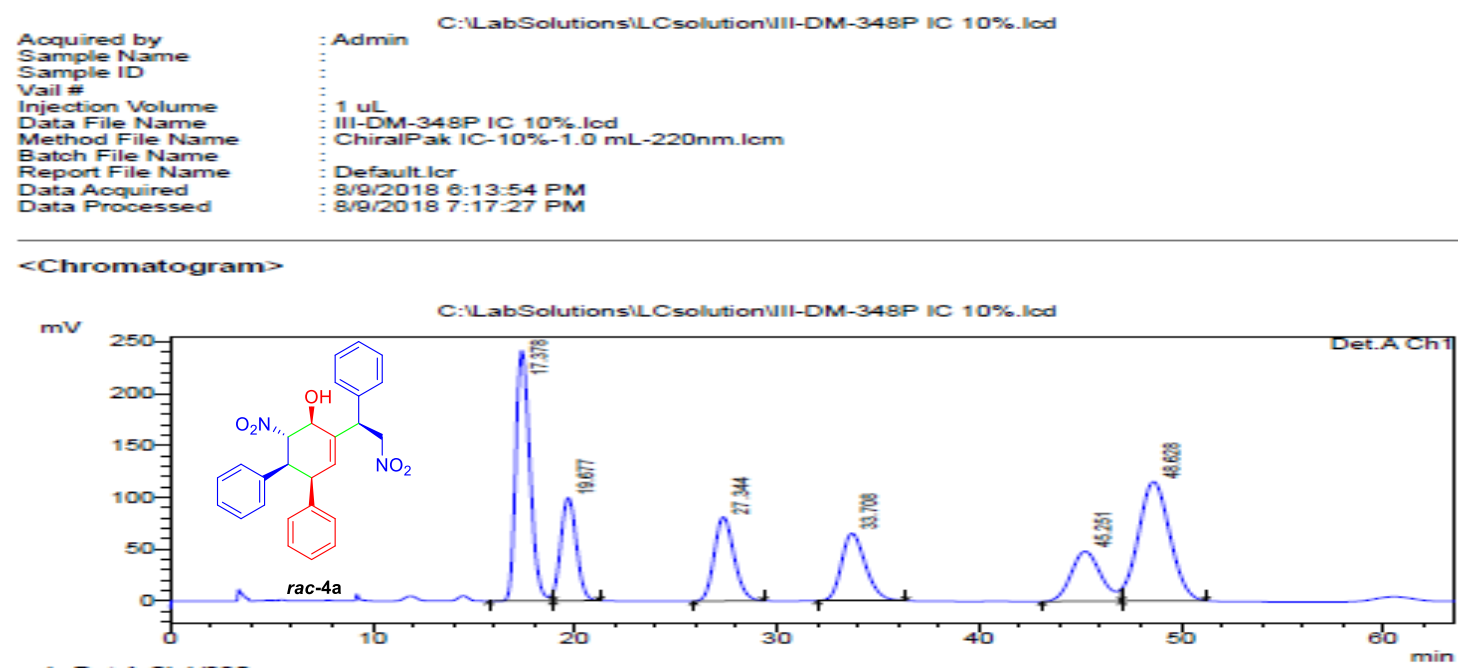

1 DetA Ch1/220nm

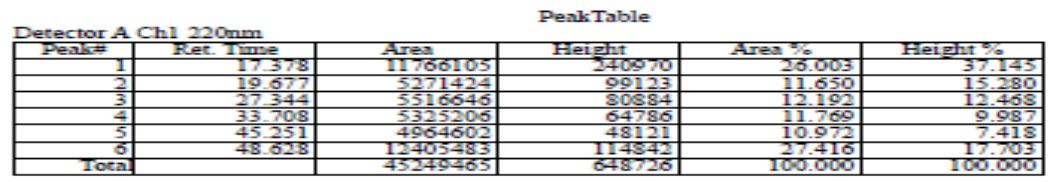

$====$ Shimadzu LCsolution Analysis Report $====$

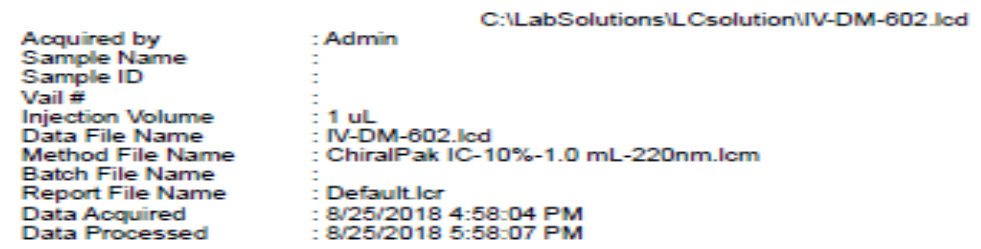

<Chromatogram>

$\mathrm{mV}$

C:MLabSolutionsL Csolution MV-DM-602 lod

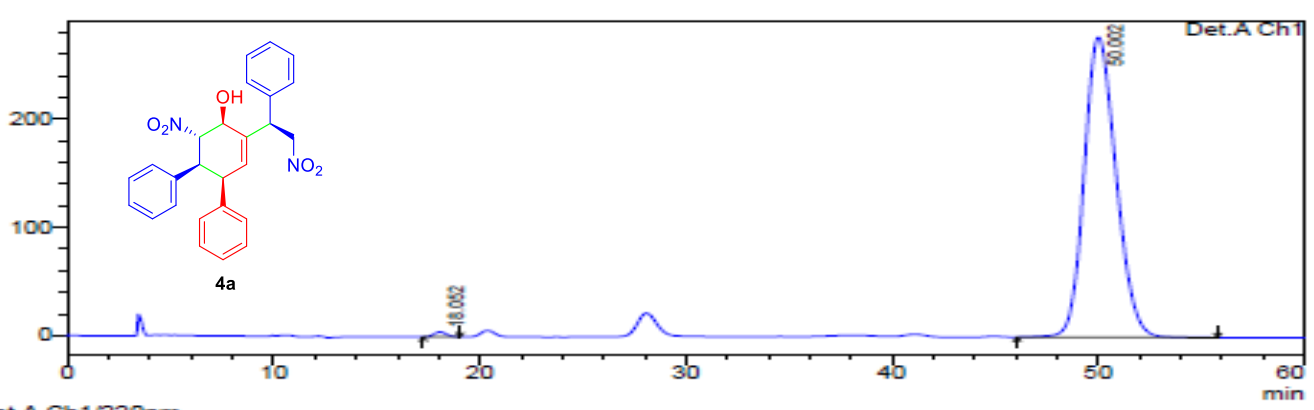

1 Det.A Ch1/220nm

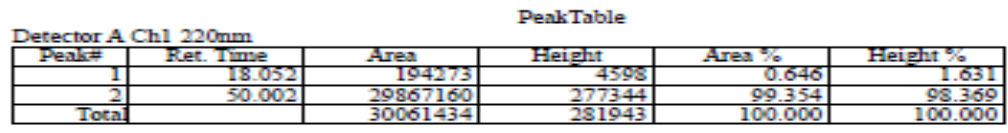




\section{$====$ Shimadzu LCsolution Analysis Report $====$}

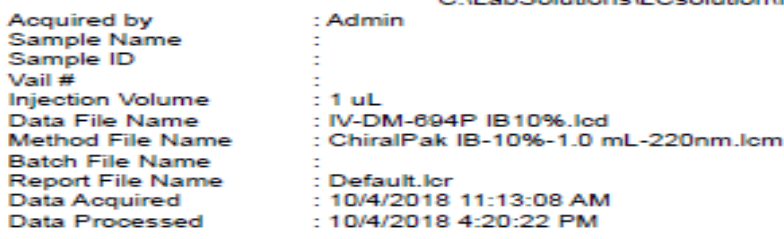

: 1 uL

IV-DM-694P IB $10 \% 6$

ChiralPak IB-10\%-1.0 mL-220nm.lcm

Default.lor

$<$ Chromatogram>

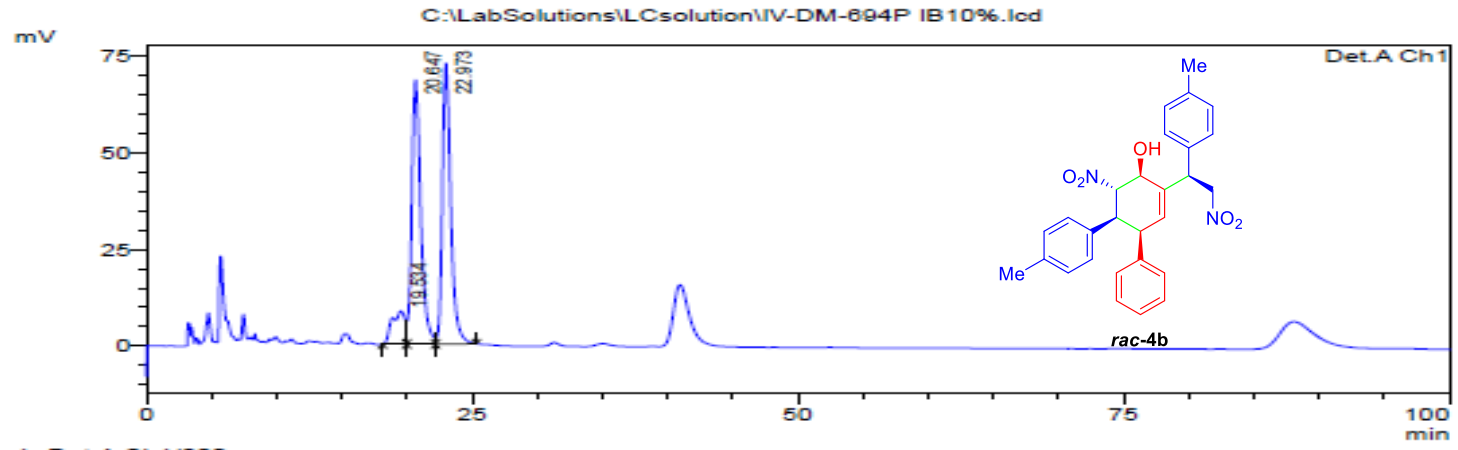

1 Det.A.Ch $1 / 220 \mathrm{~nm}$

Detector A Ch1 220nm
\begin{tabular}{|r|r|r|r|r|r|}
\hline Pealk\# & Ret. Time & Area & Height & Area \% & Height \% \\
\hline 1 & 19.534 & 587153 & 8555 & 8.254 & 5.722 \\
\hline 2 & 20.647 & 3263077 & 68319 & 45.869 & 45.693 \\
\hline 3 & 22.973 & 3263743 & 72642 & 45.878 & 48.585 \\
\hline Total & & 7113973 & 149517 & 100.000 & 100.000 \\
\hline
\end{tabular}

$===$ Shimadzu LCsolution Analysis Report $===$

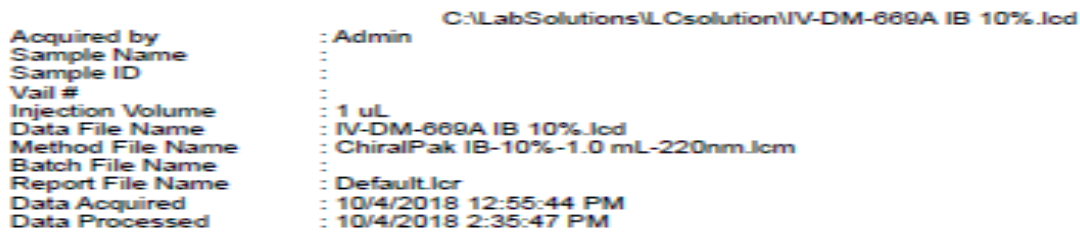

\section{$<$ Chromatogram>}

mV

C:VLabSolutionsULCsolutionXV-DM-669A IB 10\%.lod

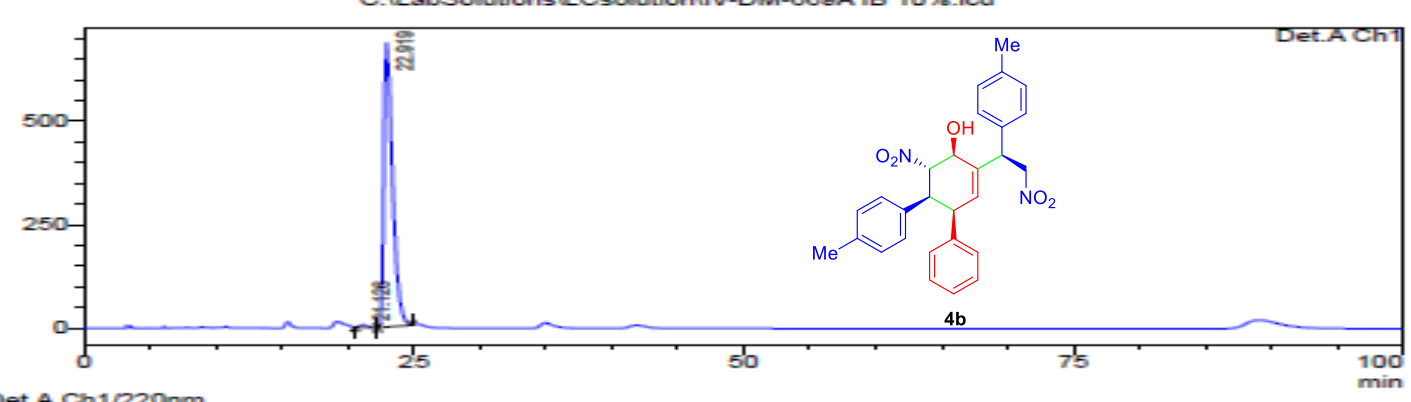

1 Det.A Ch1/220inm

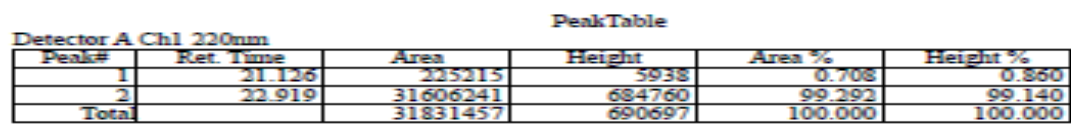




\section{$===$ Shimadzu LCsolution Analysis Report $===$}

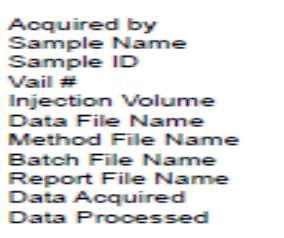

: Admin

C:NLabSolutionsLLCsolutionIV-DM-711 IC 10\%_lod

<Chromatogram>

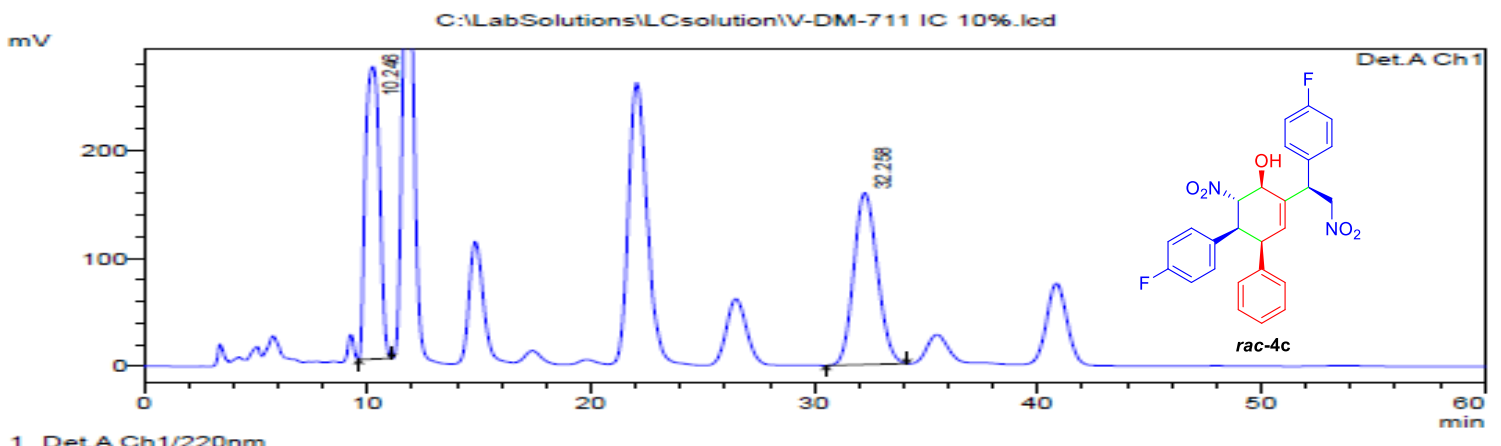

1 Det.A. Ch $1 / 220 \mathrm{~nm}$

1 uL

Y-DM-711 IC 1096 .

ChiralPak IC-1096-1.0 mL-220nm-lom

Default. lor

10/8/2018 $3: 46: 36$ PM
$10 / 8 / 2018$ 4:46:39 PM

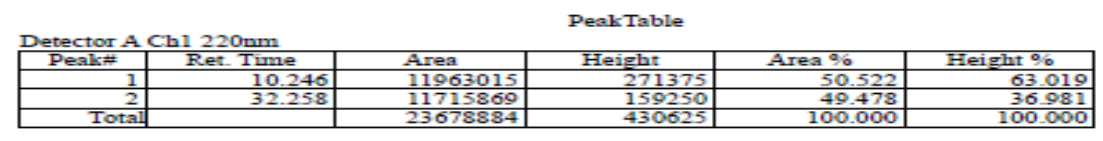

$====$ Shimadzu LCsolution Analysis Report $====$

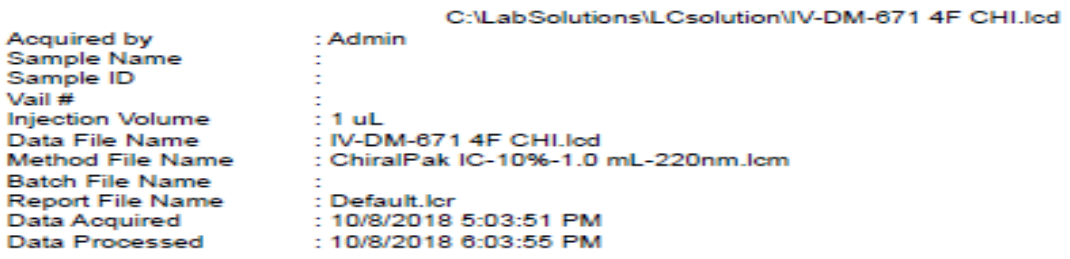

IV-DM-671 4F CHI_lod

ChiralPak IC-10\%6-1.0 mL-220nm.lom

Default.lor

5-03:51 PM

10/8/2018 6:03:55 PM

$<$ Chromatogram>

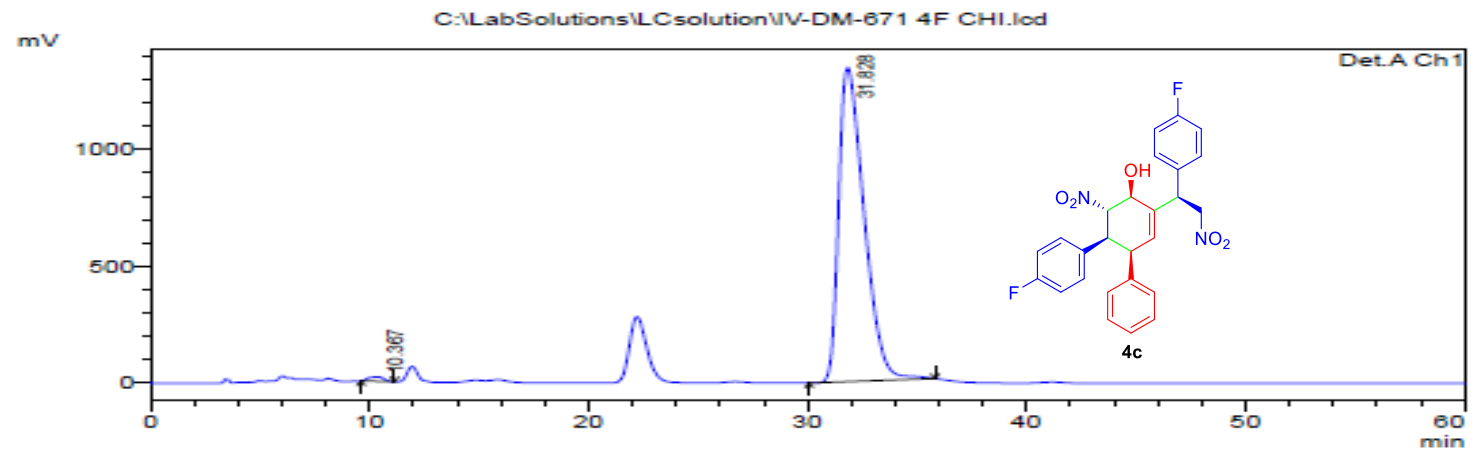

1 Det.A Ch $1 / 220 \mathrm{~nm}$

\begin{tabular}{|c|c|c|c|c|c|}
\hline \multirow{2}{*}{ Detector A } & \multicolumn{5}{|c|}{ PeakTable } \\
\hline & Ret. Time & Area & Height & Area \% & Height \% \\
\hline & $\frac{10.367}{31.828}$ & $\begin{array}{r}898940 \\
108278107\end{array}$ & $\begin{array}{r}19002 \\
1344869 \\
\end{array}$ & $\begin{array}{r}0.823 \\
99.177\end{array}$ & $\begin{array}{r}1.39 \\
98.60\end{array}$ \\
\hline Tota & & 109177047 & 1363871 & 100.000 & 100.00 \\
\hline
\end{tabular}




\section{Shimadzu LCsolution Analysis Report $====$}

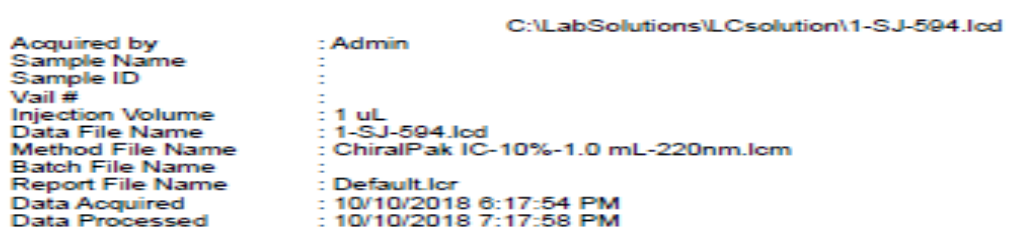

$<$ Chromatogram>

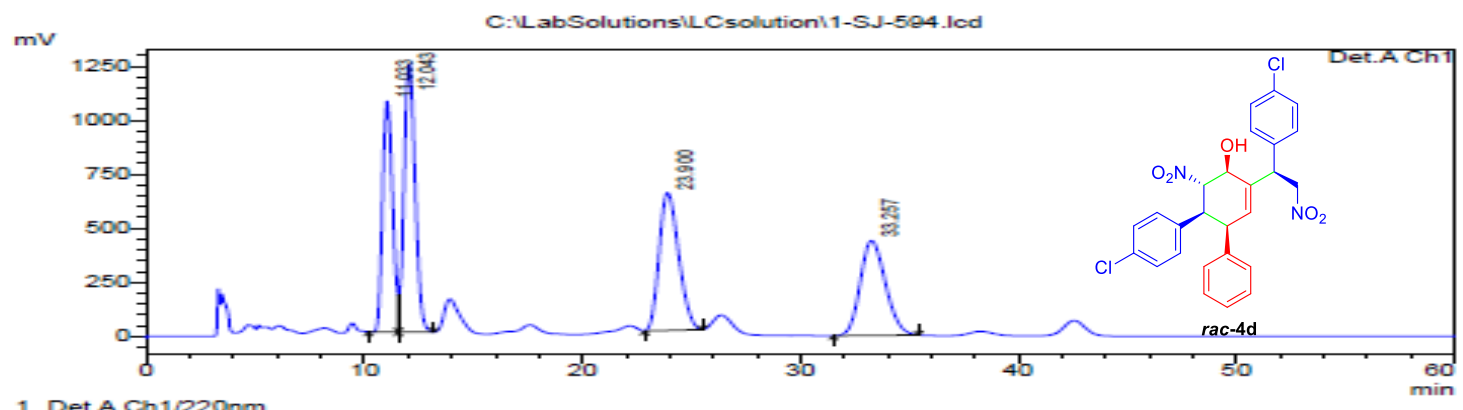

1 Det.A Ch $1 / 220 \mathrm{~mm}$

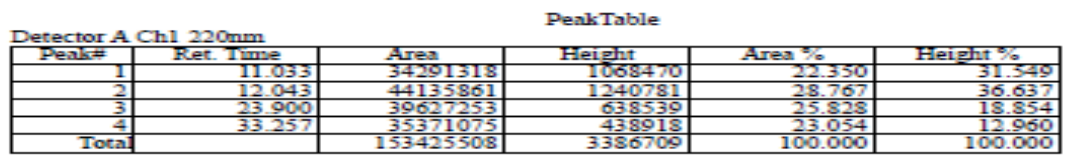

$====$ Shimadzu LCsolution Analysis Report $===$

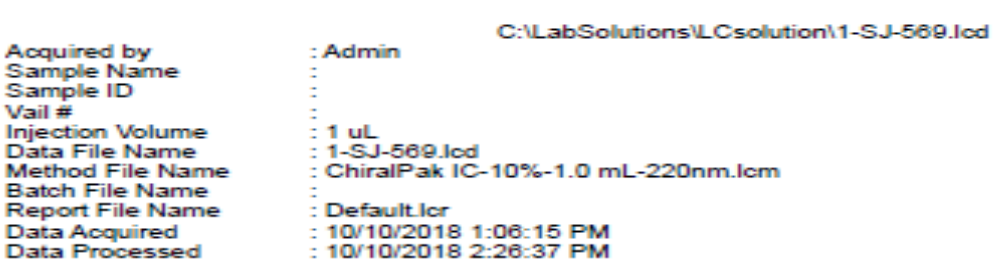

<Chromatogram>

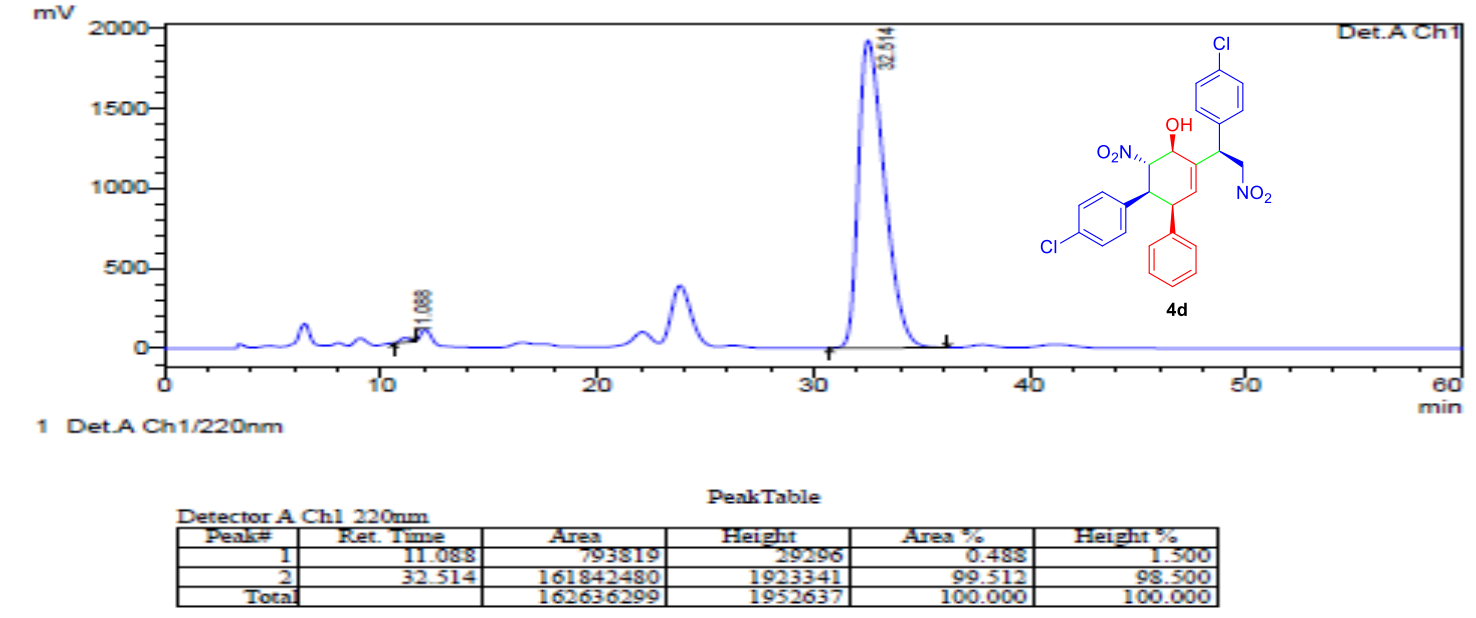

1 Det.A Ch $1 / 220 \mathrm{~nm}$ 


\section{$====$ Shimadzu LCsolution Analysis Report $===$}

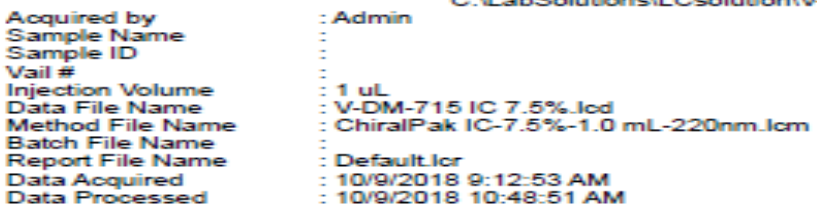

<Chromatogram>

$m v$

C:VLabSolutions LLCsolutionIV-DM-715 IC 7.5\%.lod

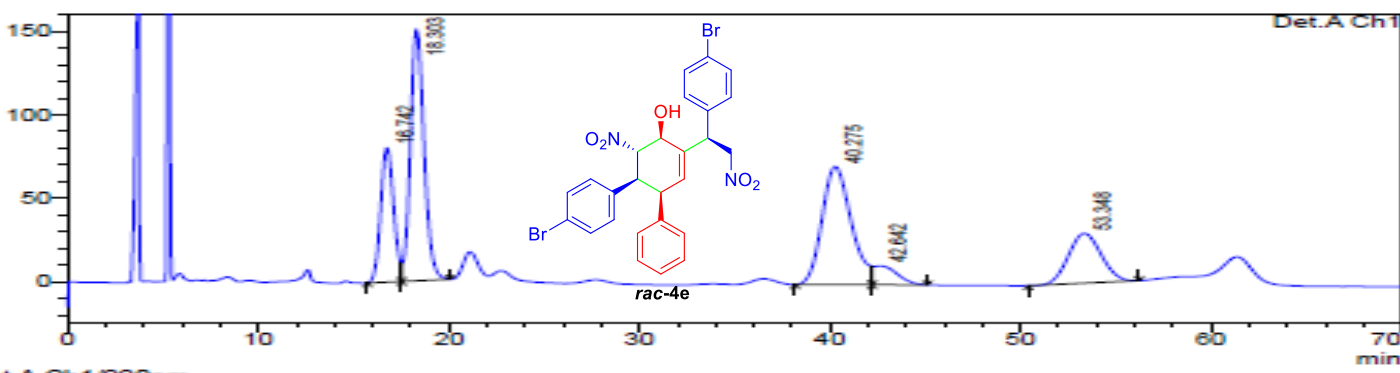

1 Det.A Ch $1 / 220 \mathrm{~mm}$

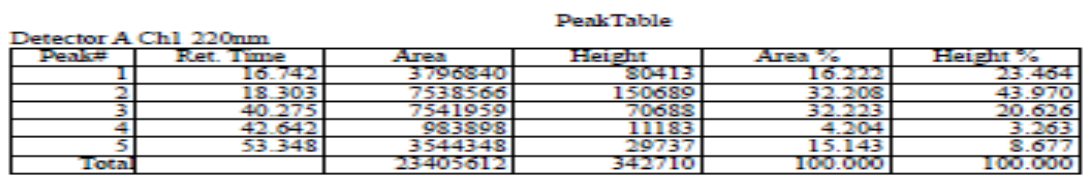

$====$ Shimadzu LCsolution Analysis Report $===$

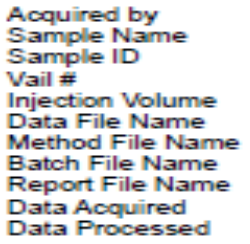

: Admin

C:MabSolutions L CsolutionUV-DM-S573.llod

$\vdots$

1 uL

$5573 \mathrm{lcd}$

ChiralPak $1 C-7.5 \%-1.0 \mathrm{~mL}-220 \mathrm{~nm} . \mathrm{lam}$

Default lor

$10 / 9 / 2018$ 10:45:37 AM

10 rg/2018 12:08-29 PM

$<$ Chromatogram>

$\mathrm{mV}$

C:VabSolutions'LCsolutionIV-DM-S573 lod

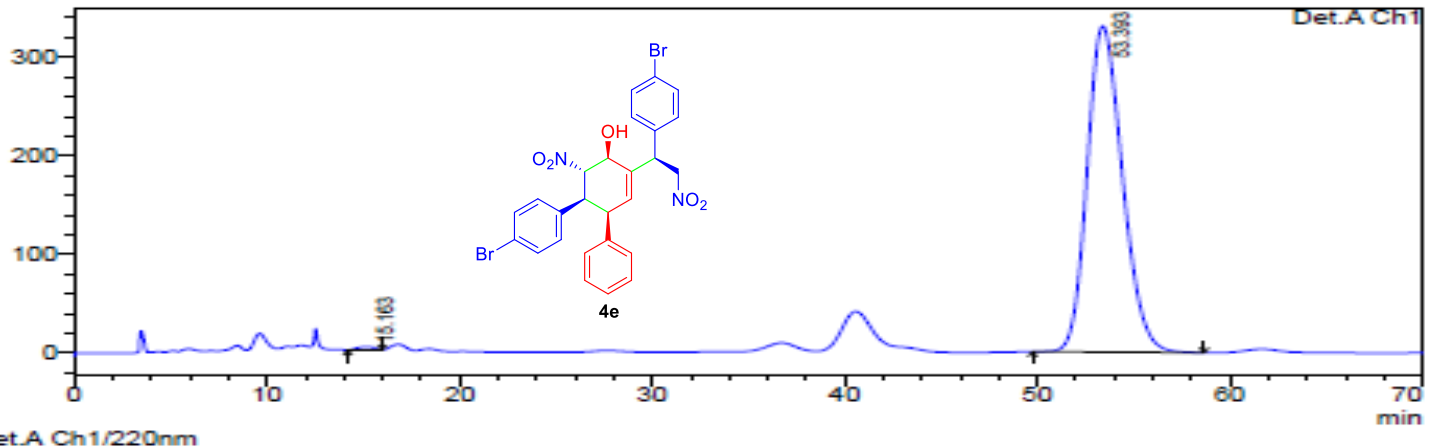

1 Det.A Ch1/220nm

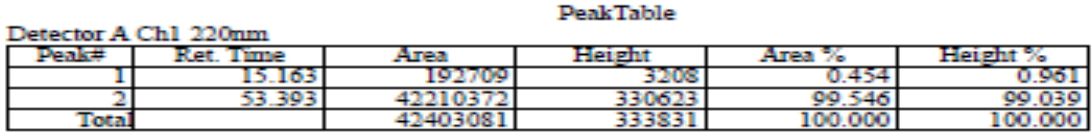




\section{$===$ Shimadzu LCsolution Analysis Report $====$}

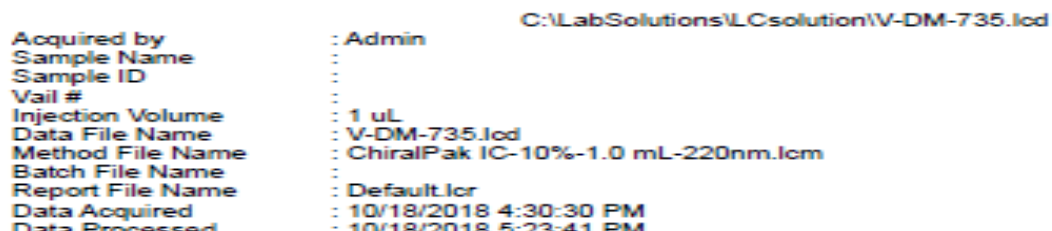

<Chromatogram>

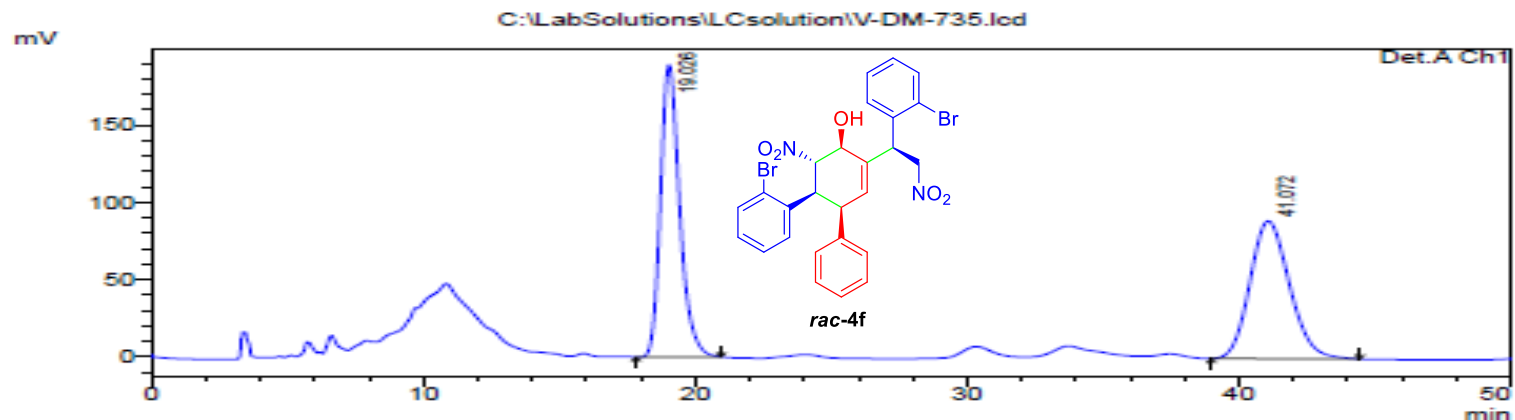

1 Det.A. Ch1/220mm

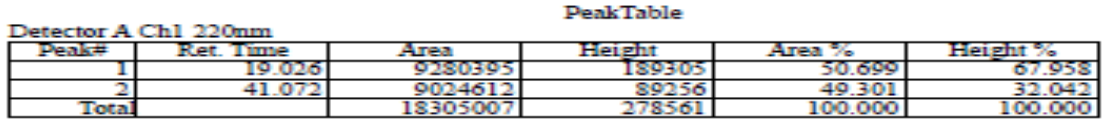

$====$ Shimadzu LCsolution Analysis Report $===$

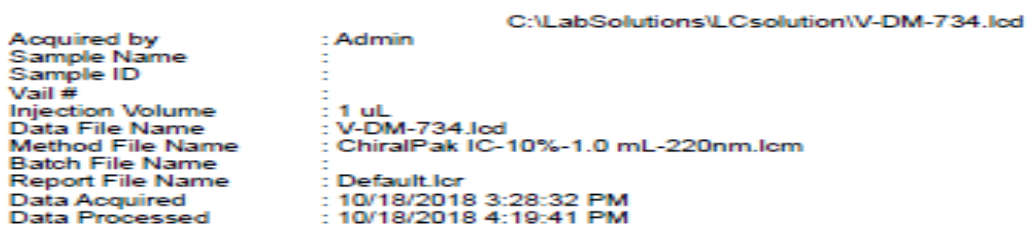

\section{<Chromatogram>}

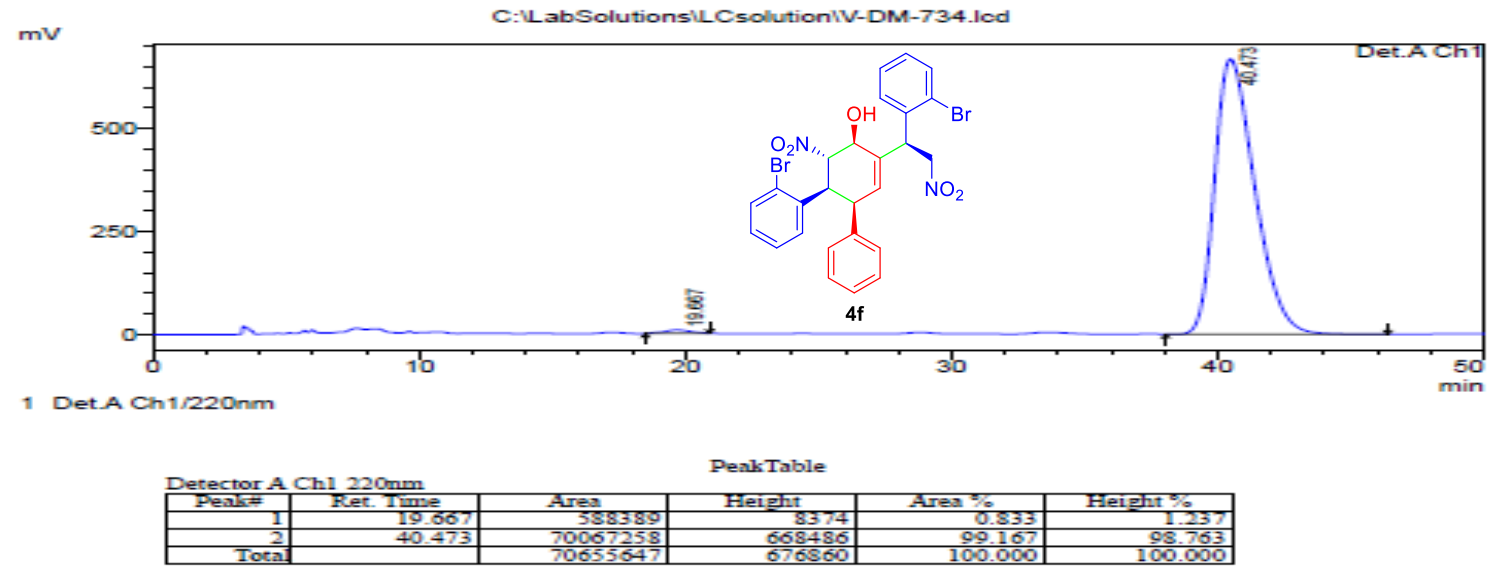



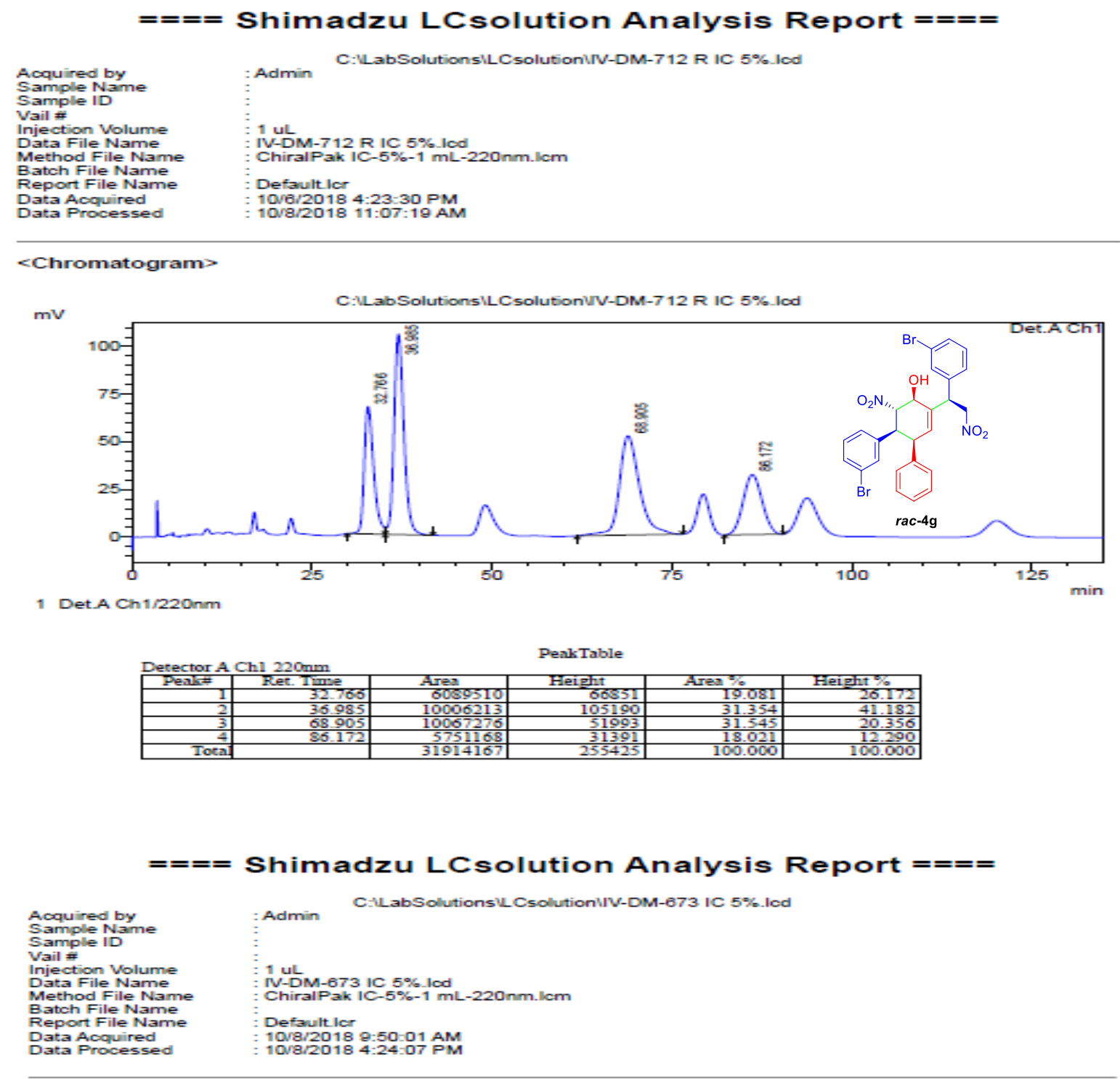

<Chromatogram>

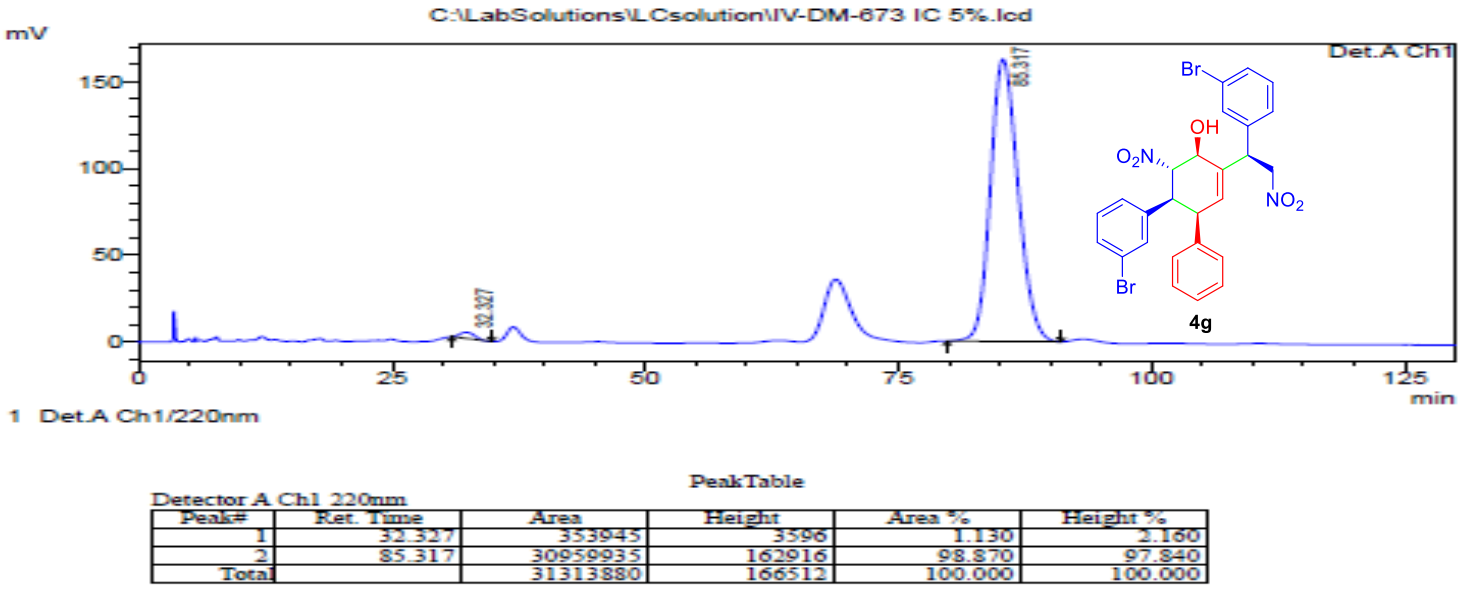




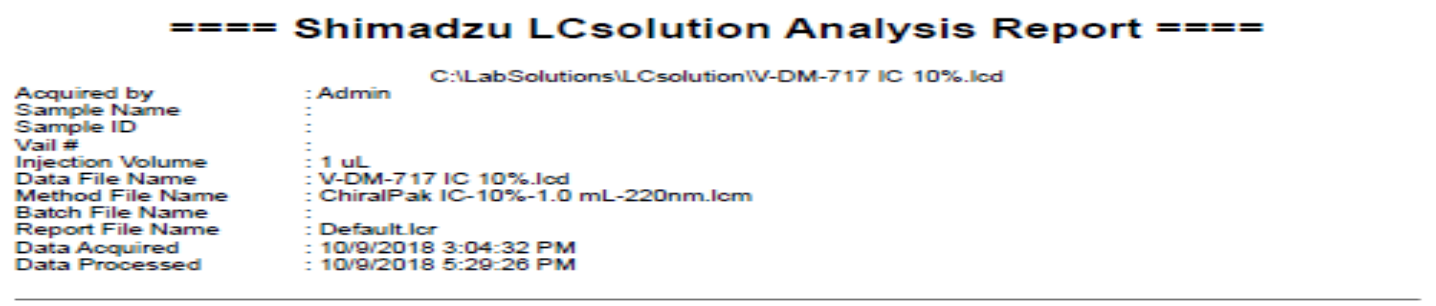

<Chromatogram>

mV C:NLabSolutionsUCSolutionV-DM-717 IC 10\%.lcd

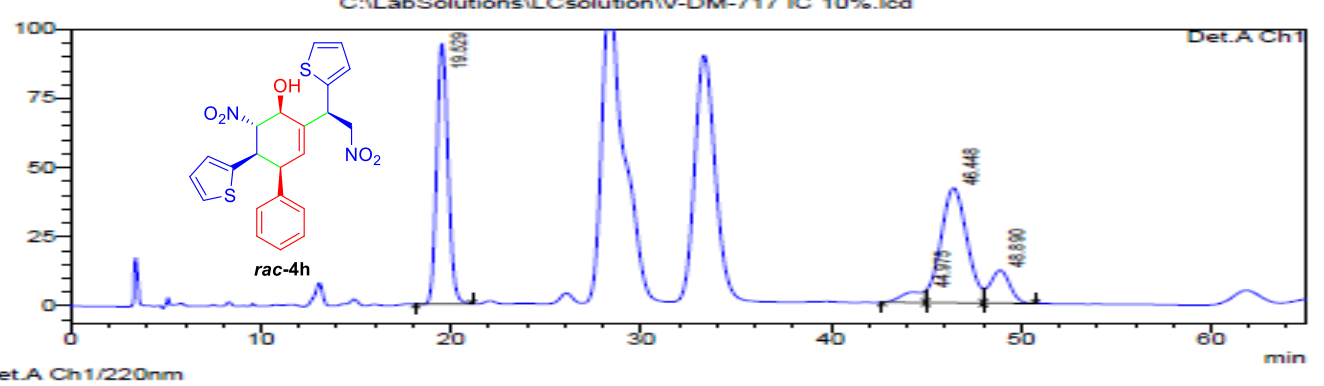

1 Det.A Ch1/220nm

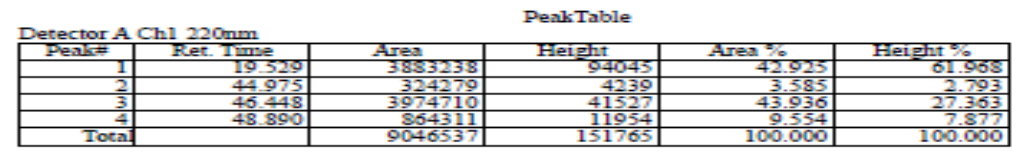

$====$ Shimadzu LCsolution Analysis Report $===$

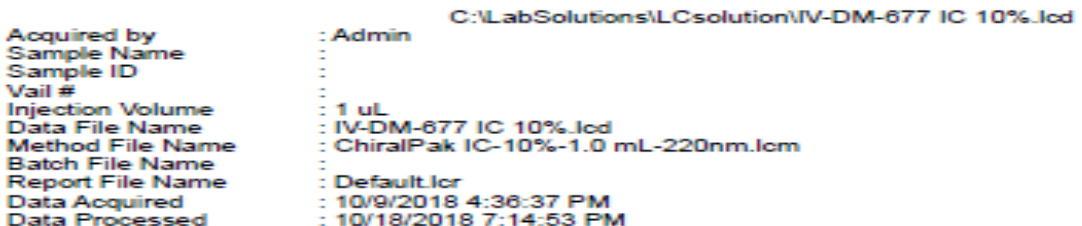

<Chromatogram>

mv

C:MabSolutions LLCsolutionUV-DM-677 IC 10\%.lod

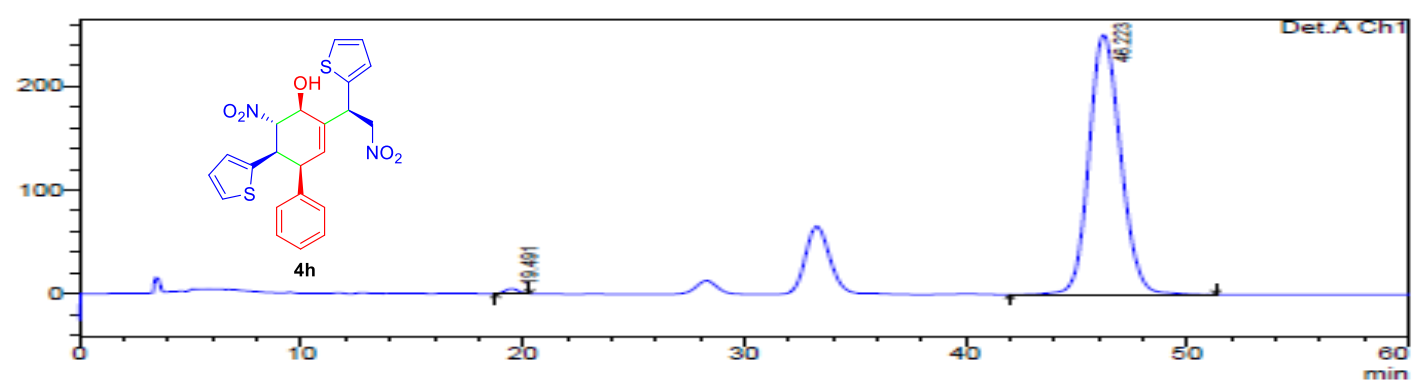

1 Det.A Ch1/220nm

\begin{tabular}{|c|c|c|c|c|c|}
\hline \multicolumn{6}{|c|}{ Detector A Chl $220 \mathrm{~mm}$} \\
\hline peals $\#$ & Ret. Time & Arrea & Height & Arean $\%$ & Heifht \% \\
\hline & 19.491 & 191888 & 4634 & 0.765 & 1.815 \\
\hline 2 & 46.223 & $\frac{24900111}{25007001}$ & $\frac{250692}{755230}$ & $\frac{99.235}{0.090}$ & $\frac{98.185}{00.000}$ \\
\hline
\end{tabular}




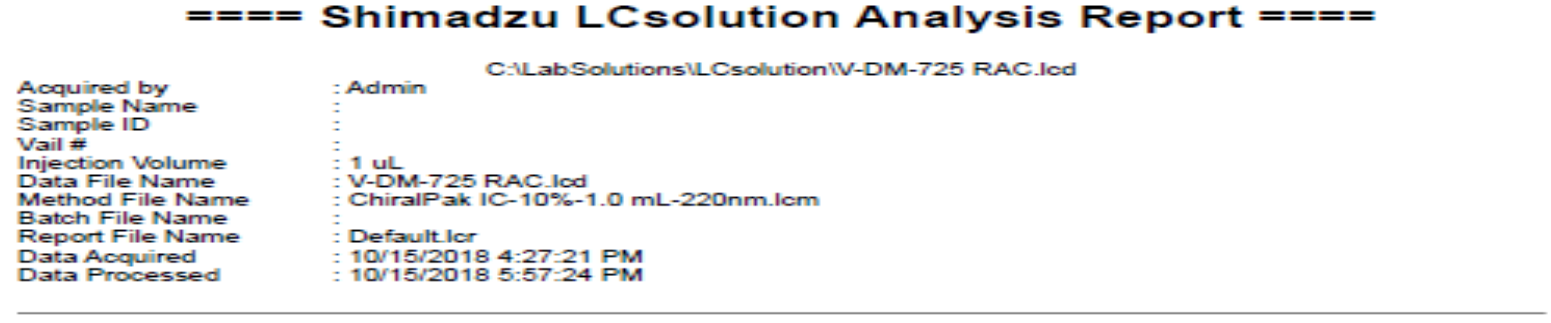

\section{<Chromatogram>}

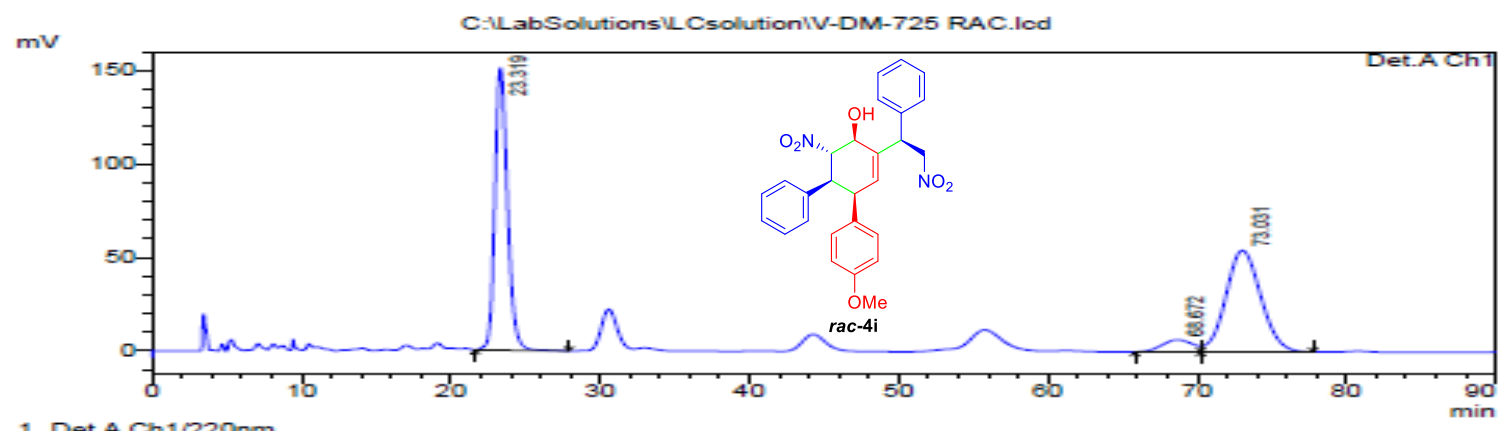

1 Det.A Ch1/220nm

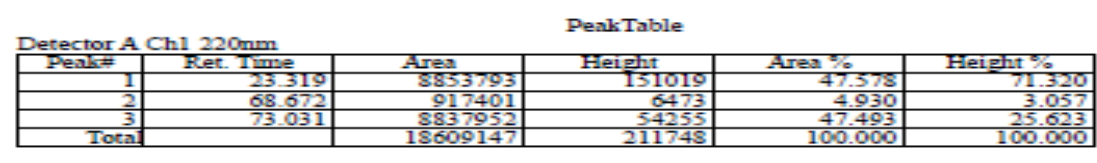

$===$ Shimadzu LCsolution Analysis Report $===$

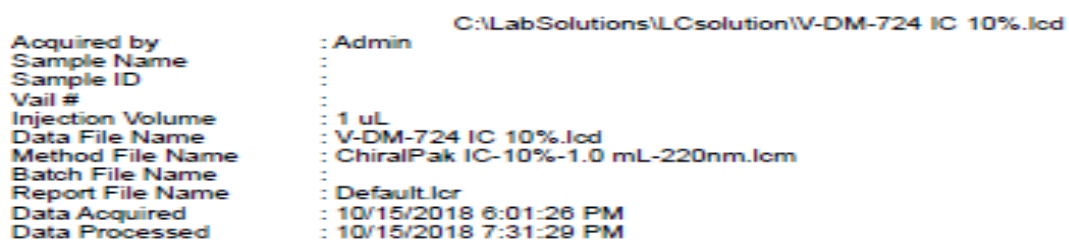

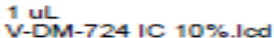

ChiralPak IC-10\%-1.0 mL-220nm.lom

Default lor

10/15/2018 6:01:26 PM

$\mathrm{mV}$

C:NLabSolutions LC SolutionW-DM-724 IC 10\%.lod

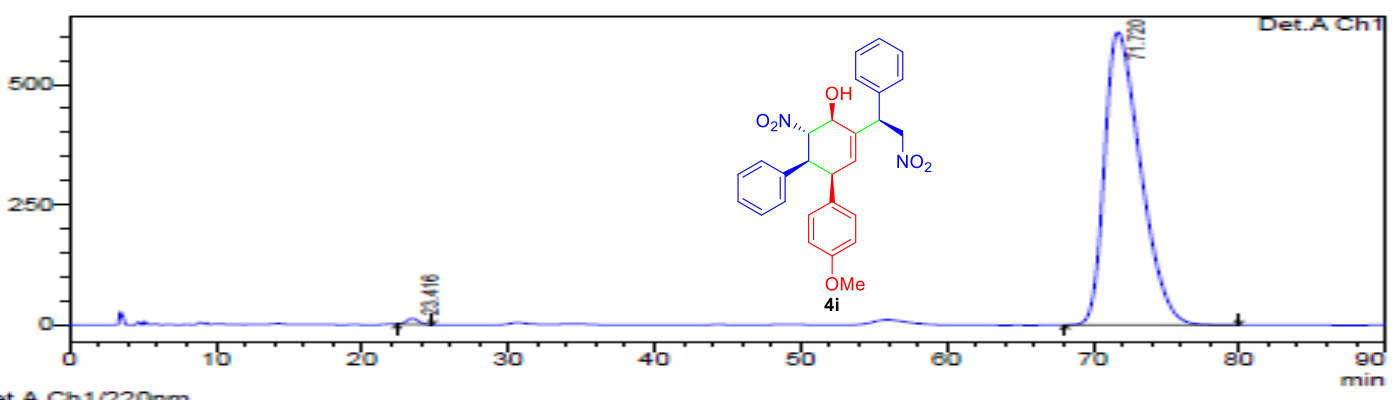

1 Det.A Ch1/220nm

\section{PeakTable}

\begin{tabular}{|c|c|c|c|c|c|}
\hline \multicolumn{6}{|l|}{ et } \\
\hline Peak $=$ & Ret. In⿴囗丨丿㇄口e & Arrea & Height & & F \\
\hline & 23.416 & 679467 & 12187 & 0.647 & 1.962 \\
\hline Toca & 71.720 & $\frac{104407112}{105086579}$ & \begin{tabular}{r|}
608993 \\
621181
\end{tabular} & $\begin{array}{r}99.353 \\
100.000\end{array}$ & $\begin{array}{l}98.038 \\
100.000\end{array}$ \\
\hline
\end{tabular}


$===$ Shimadzu LCsolution Analysis Report $===$

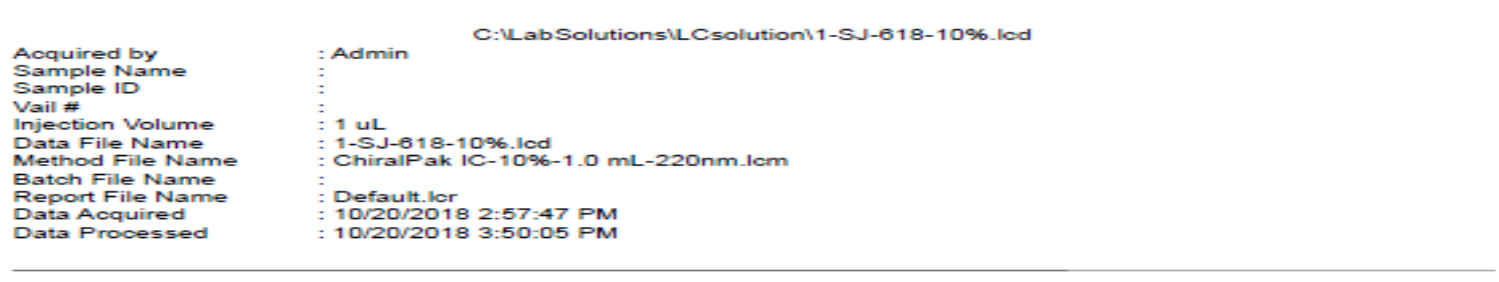

<Chromatogram>

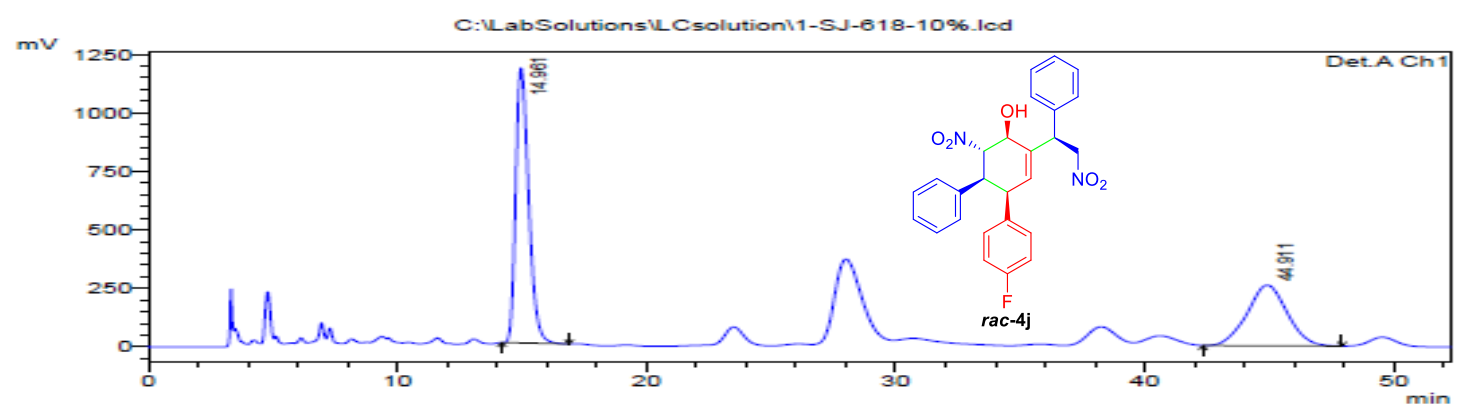

1 Det.A Ch $1 / 220 \mathrm{~nm}$

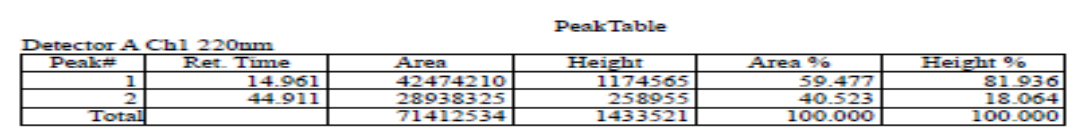

$===$ Shimadzu LCsolution Analysis Report $===$
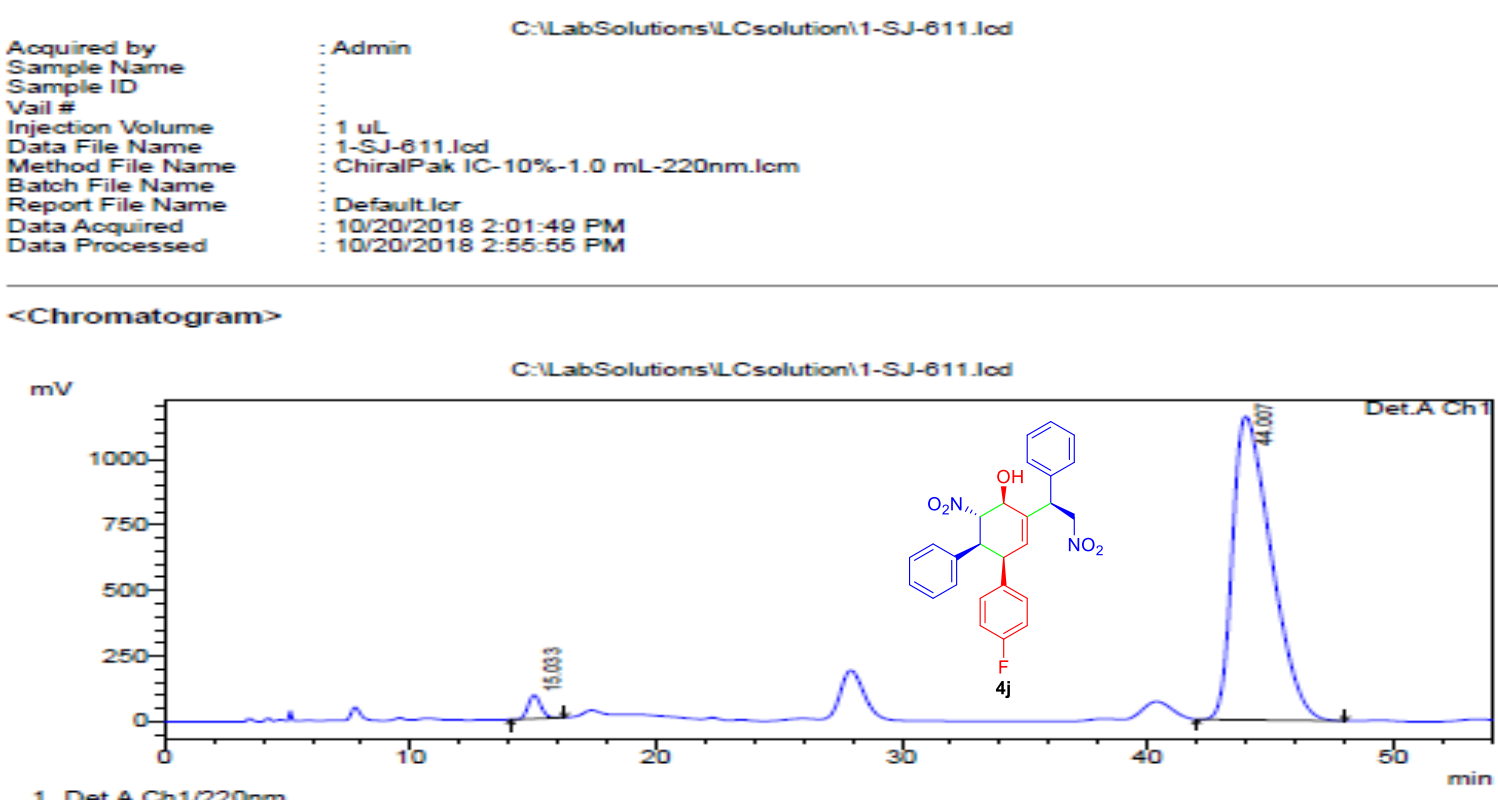

1 Det.A Ch1/220nm

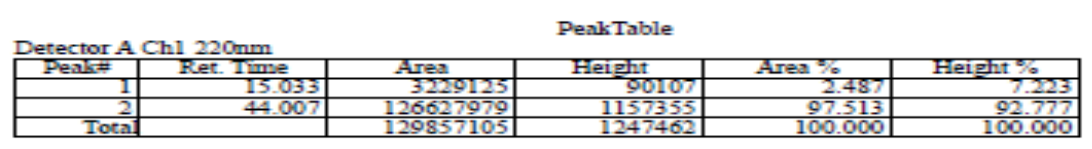


$===$ Shimadzu LCsolution Analysis Report $===$

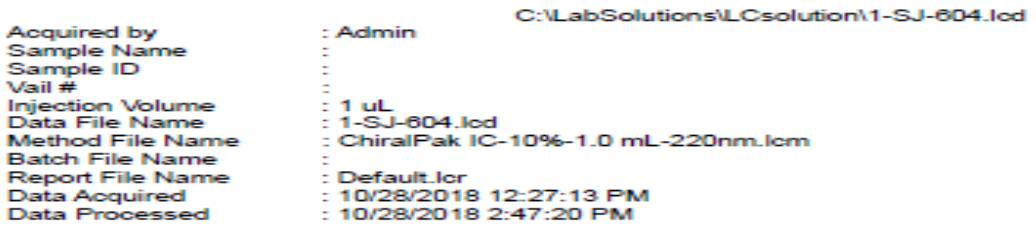

1 uL

1-SJ-604.lod $10 \% 5-1.0 \mathrm{~mL}-220 \mathrm{~mm}$.lom

Chiralpak 1 (

Default lor

$10 / 28 / 2018$ 12:27:13 PM
$10 / 28 / 2018$ 2:47:20 PM

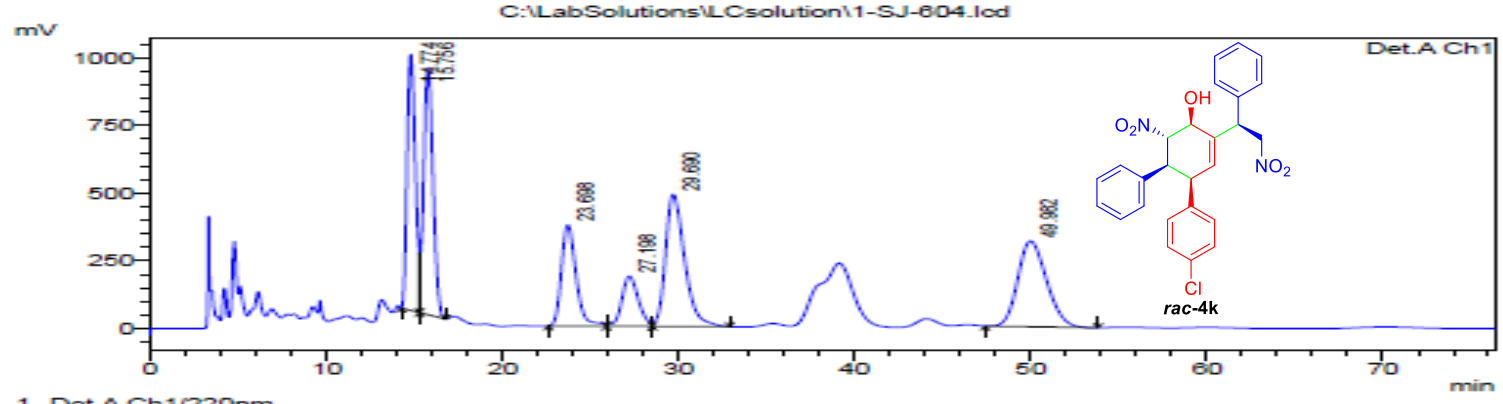

1 Det.A Ch1/220nm

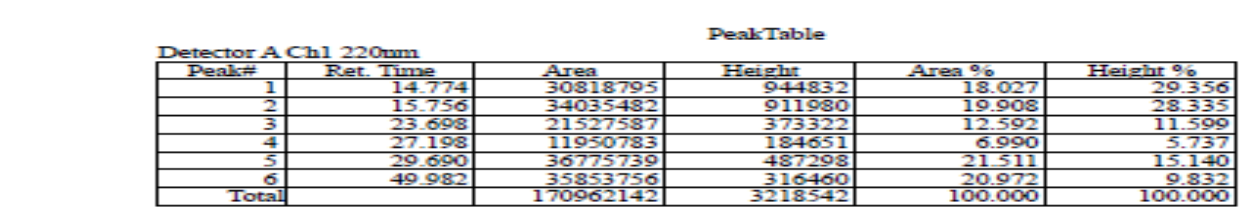

\section{$====$ Shimadzu LCsolution Analysis Report $===$}
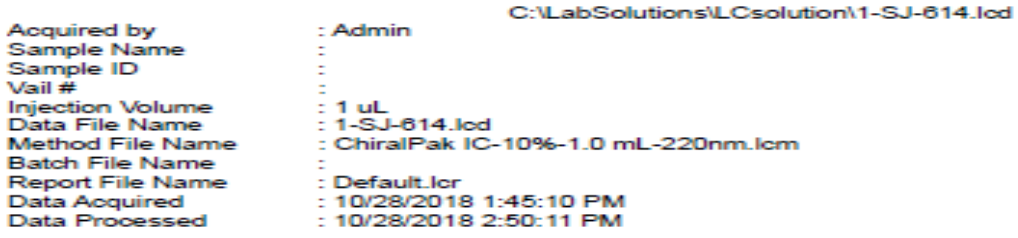

<Chromatogram>

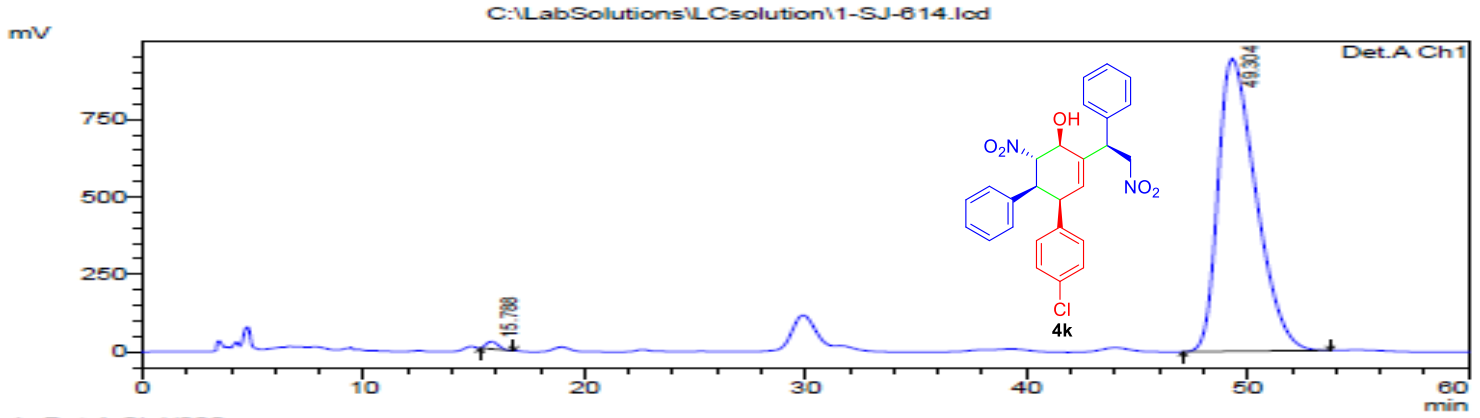

1 Det.A Ch $1 / 220 \mathrm{~nm}$

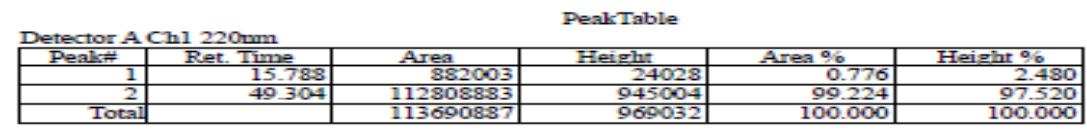




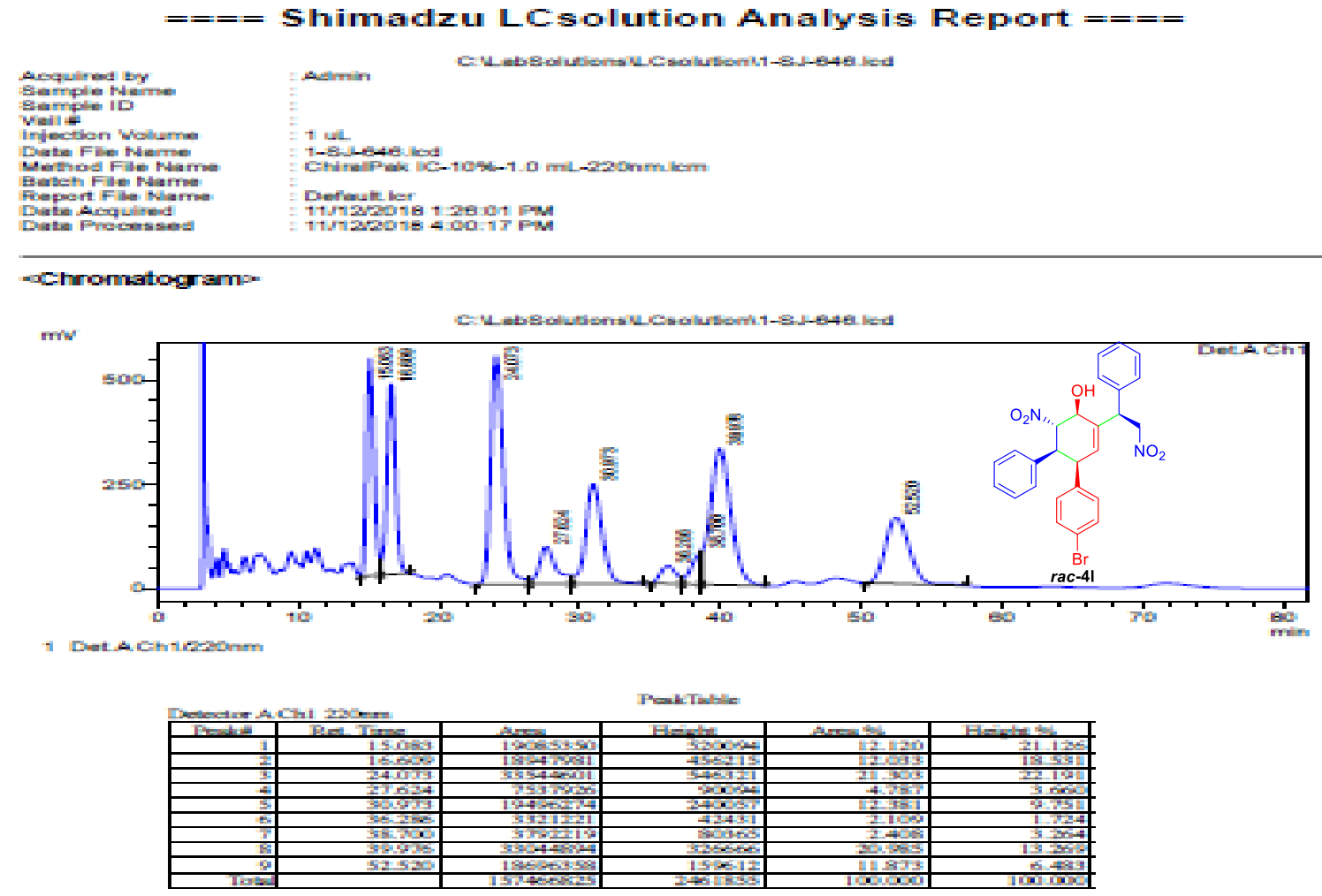

$====$ Shimadzu LCsolution Amalysis Report ===

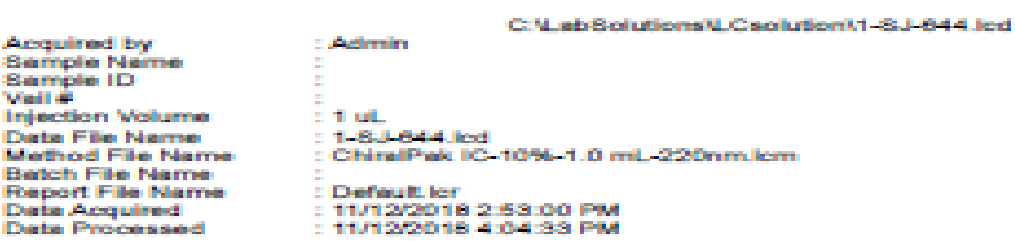

7. 1.15

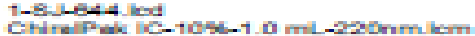

Deflaukiti

71Magsil 2 -

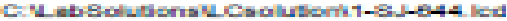

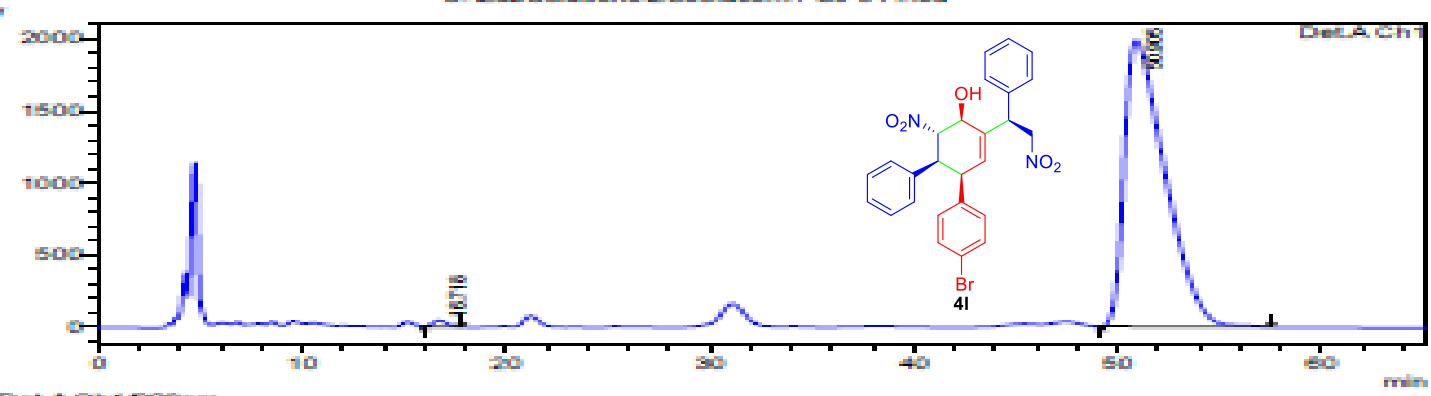

1 Dat.AChiresonm 


\section{$====$ Shimadzu LCsolution Analysis Report $===$}

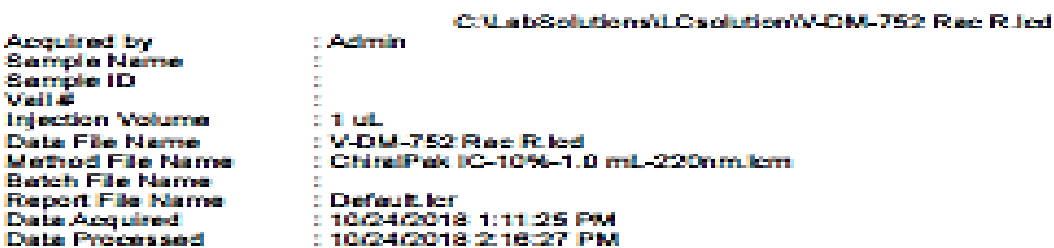

- Chromatogram:

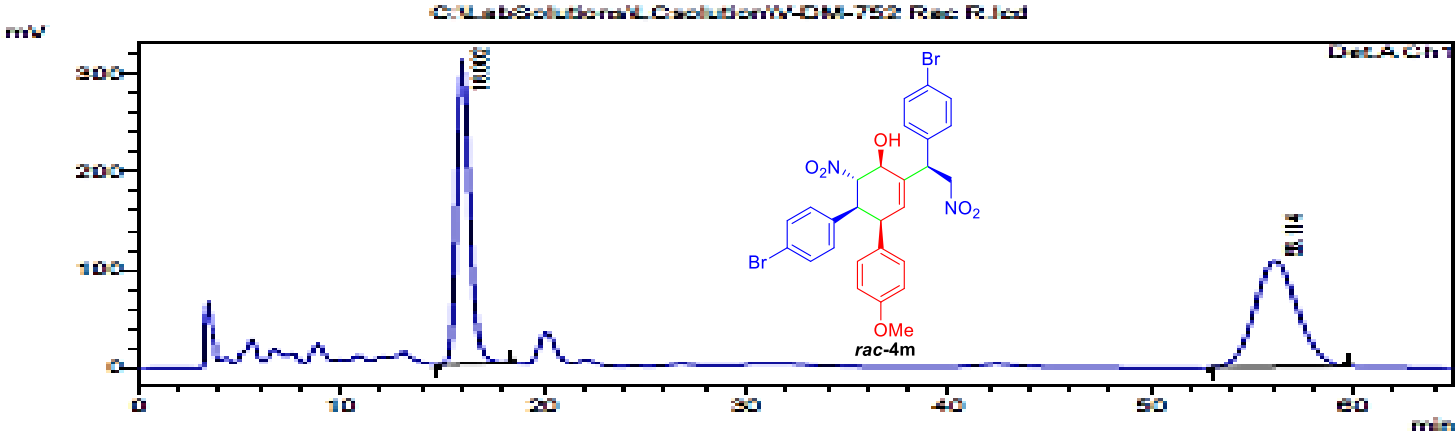

1 DeLAChiconet

\begin{tabular}{|c|c|c|c|c|c|}
\hline \multicolumn{6}{|c|}{ Domedrachl thorr } \\
\hline & Fen Tres & $\frac{\operatorname{ancs}}{1+2+64}$ & H=rht & 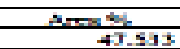 & 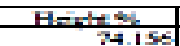 \\
\hline & 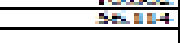 & 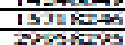 & 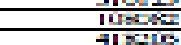 & $2 \pm+5$ & 3isnd \\
\hline
\end{tabular}

\section{$===$ Shimadzu LCsolmtion Analysis Report ===}

Antitimi tr Eampin thame

Eartiple ic

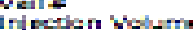

Dit Fil the

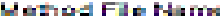

Eabeti Fil than

Fersin Fil than

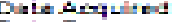

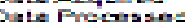

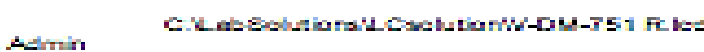

Chromatogram:

$m$

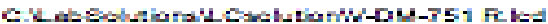

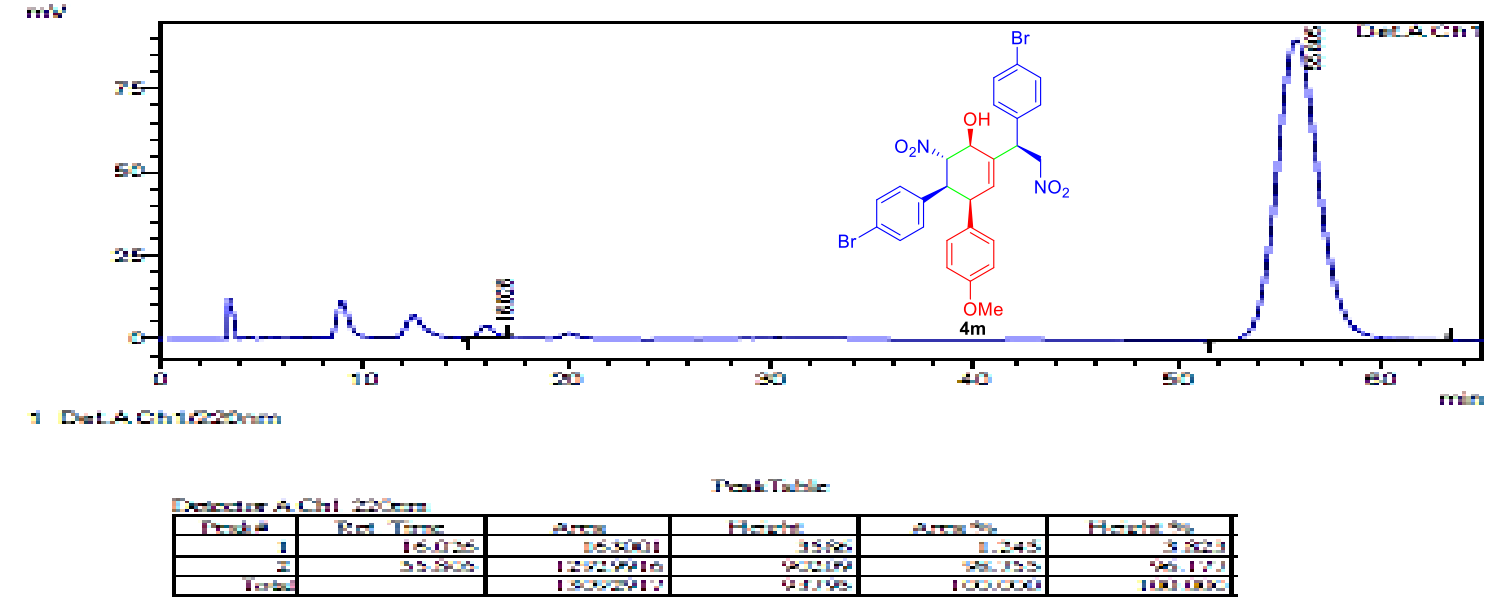

1 DeLachigrowh

$1+L$

THOT F

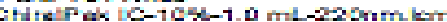

Defiater

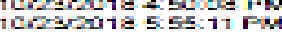




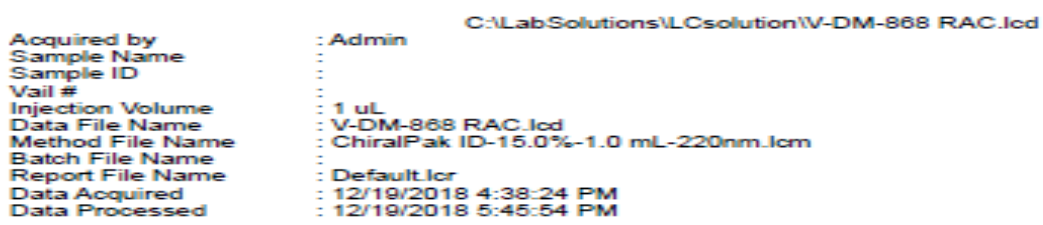

<Chromatogram>

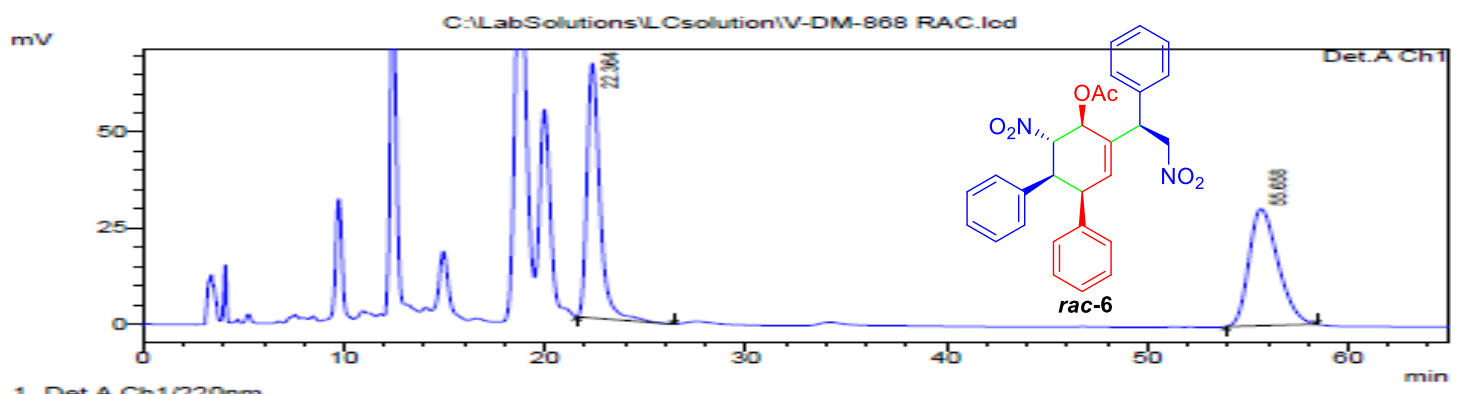

1 Det.A Ch1/220nm
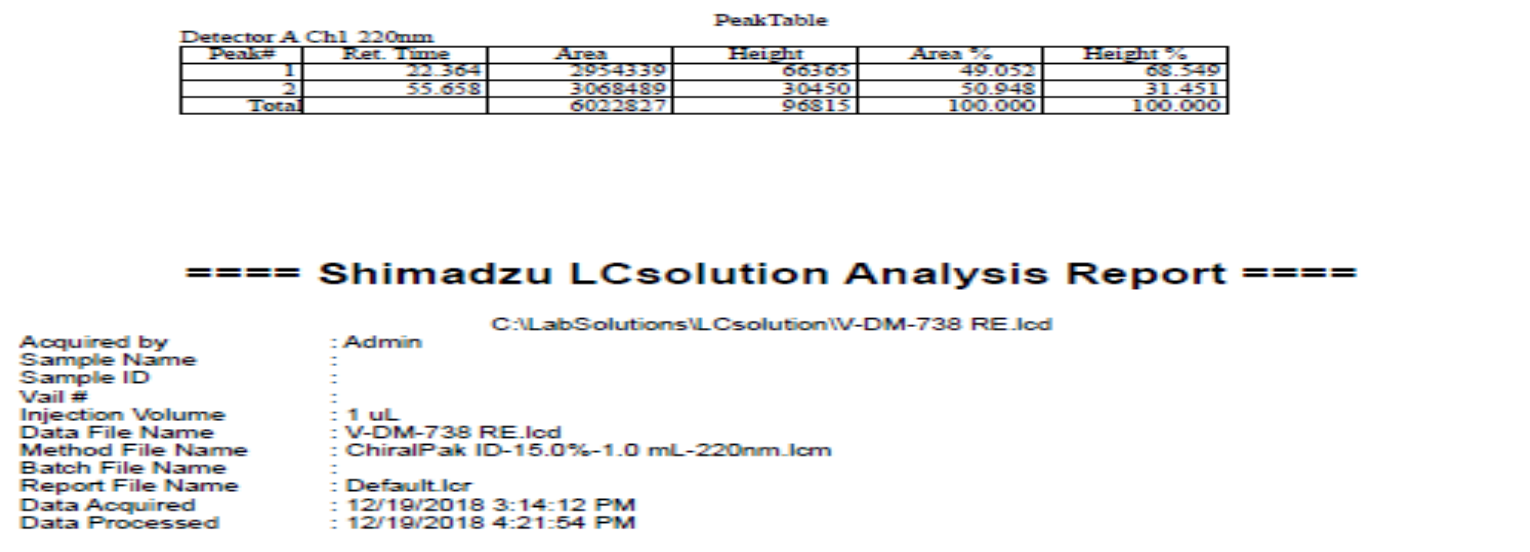

<Chromatogram>

$\mathrm{mV}$

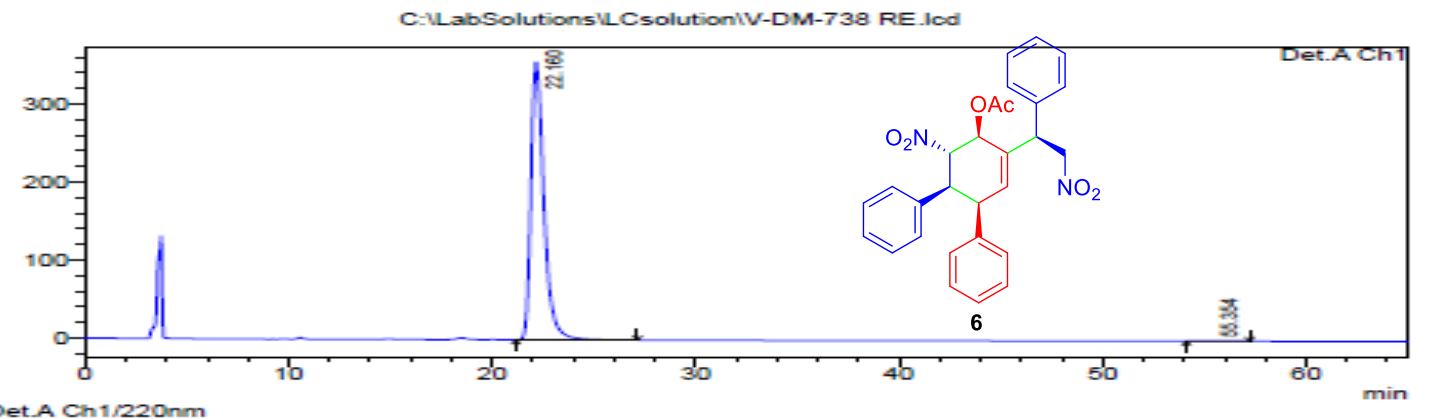

1 DetACh1/220nm

\begin{tabular}{|c|c|c|c|c|c|}
\hline Det & \multicolumn{5}{|c|}{ eak Table } \\
\hline Peank & RLet. Time & Arrea & Height & Arrea \% & Height \% \\
\hline & $\frac{22.160}{55.354}$ & $\frac{15855045}{29215}$ & $\frac{350185}{333}$ & $\frac{99.816}{0.184}$ & $\frac{99.907}{0.093}$ \\
\hline$\overline{T c}$ & & 15884261 & 356518 & 100 . & 100.000 \\
\hline
\end{tabular}



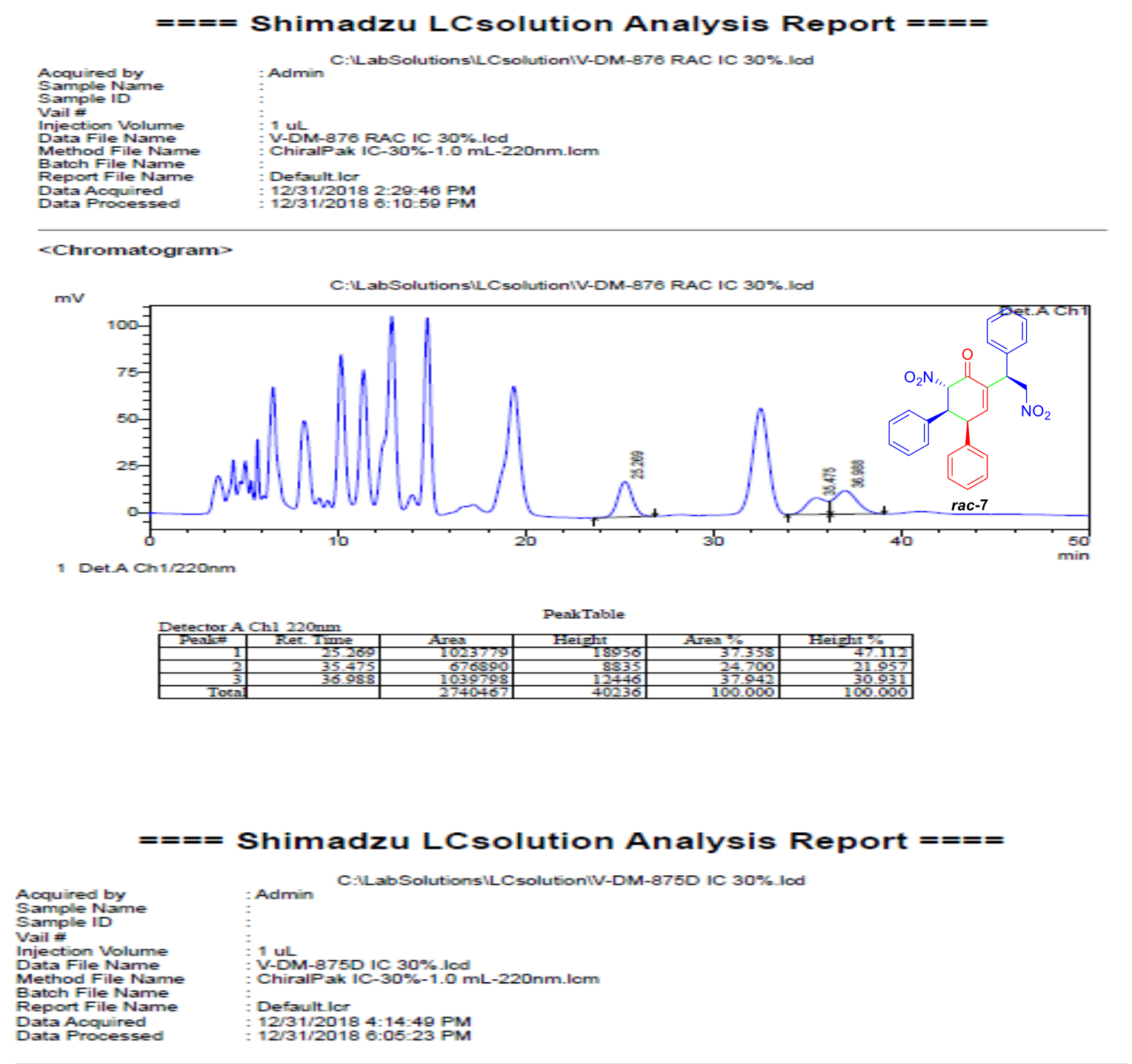

<Chromatogram>

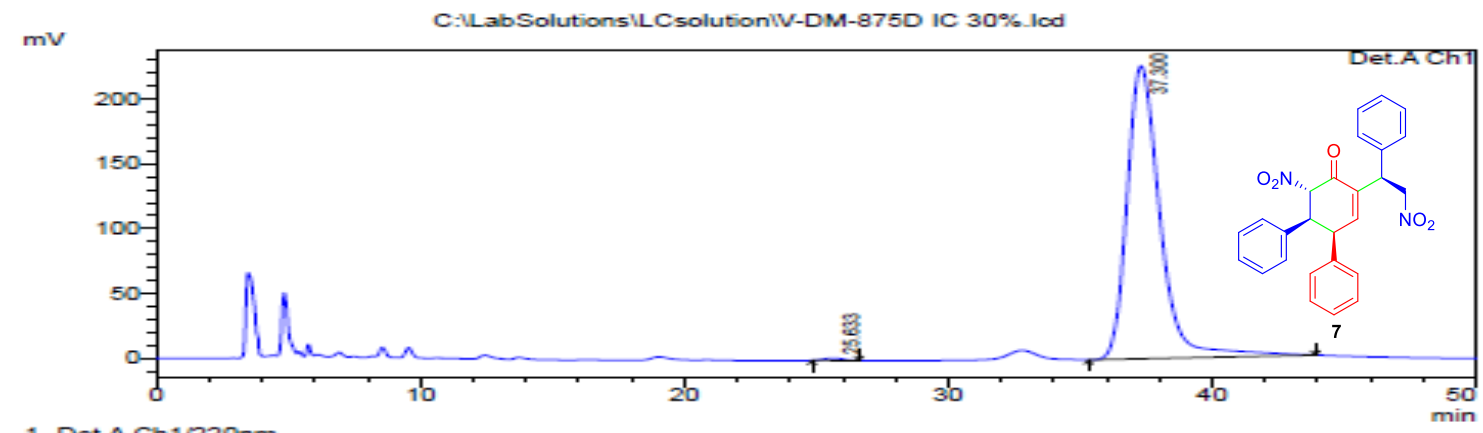

1 Det.A Ch1/220nm

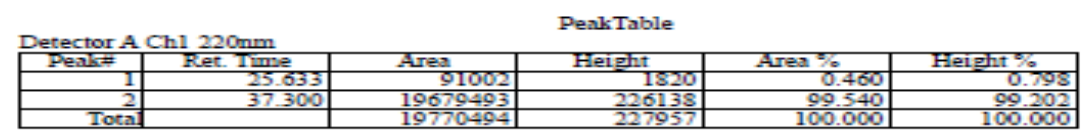

